\title{
Local Uniqueness and Refined Spike Profiles of Ground States for Two-Dimensional Attractive Bose-Einstein Condensates
}

\author{
Yujin Guo*, Changshou Lin $†$ and Juncheng Wei ${ }^{\ddagger}$
}

July 4, 2018

\begin{abstract}
We consider ground states of two-dimensional Bose-Einstein condensates in a trap with attractive interactions, which can be described equivalently by positive minimizers of the $L^{2}$-critical constraint Gross-Pitaevskii energy functional. It is known that ground states exist if and only if $a<a^{*}:=\|w\|_{2}^{2}$, where $a$ denotes the interaction strength and $w$ is the unique positive solution of $\Delta w-w+w^{3}=0$ in $\mathbb{R}^{2}$. In this paper, we prove the local uniqueness and refined spike profiles of ground states as $a \nearrow a^{*}$, provided that the trapping potential $h(x)$ is homogeneous and $H(y)=\int_{\mathbb{R}^{2}} h(x+y) w^{2}(x) d x$ admits a unique and non-degenerate critical point.
\end{abstract}

Keywords: Bose-Einstein condensation; spike profiles; local uniqueness; Pohozaev identity.

\section{Introduction}

The phenomenon of Bose-Einstein condensation (BEC) has been investigated intensively since its first realization in cold atomic gases, see [1, 5] and references therein. In these experiments, a large number of (bosonic) atoms are confined to a trap and cooled to very low temperatures. Condensation of a large fraction of particles into the same one-particle state is observed below a critical temperature. These Bose-Einstein condensates display various interesting quantum phenomena, such as the critical-mass collapse, the superfluidity and the appearance of quantized vortices in rotating traps (e.g. [5]). Specially, if the force between the atoms in the condensates is attractive, the system collapses as soon as the particle number increases beyond a critical value, see, e.g., [23] or [5, Sec. III.B].

Bose-Einstein condensates (BEC) of a dilute gas with attractive interactions in $\mathbb{R}^{2}$ can be described $([2,5,10])$ by the following Gross-Pitaevskii (GP) energy functional

$$
E_{a}(u):=\int_{\mathbb{R}^{2}}\left(|\nabla u|^{2}+V(x)|u|^{2}\right) d x-\frac{a}{2} \int_{\mathbb{R}^{2}}|u|^{4} d x,
$$

${ }^{*}$ Wuhan Institute of Physics and Mathematics, Chinese Academy of Sciences, P.O. Box 71010, Wuhan 430071, P. R. China. Email: yjguo@wipm.ac.cn. Y. J. Guo is partially supported by NSFC grants 11322104 and 11671394.

${ }^{\dagger}$ Taida Institute of Mathematical Sciences, National Taiwan University, Taipei 10617, Taiwan. Email: cslin@math.ntu.edu.tw.

${ }^{\ddagger}$ Department of Mathematics, University of British Columbia, Vancouver, BC V6T 1Z2, Canada. Email: jcwei@math.ubc.ca. J.C. Wei is partially supported by NSERC of Canada. 
where $a>0$ describes the strength of the attractive interactions, and $V(x) \geq 0$ denotes the trapping potential satisfying $\lim _{|x| \rightarrow \infty} V(x)=\infty$. As addressed recently in [10, 11], ground states of attractive BEC in $\mathbb{R}^{2}$ can be described by the constraint minimizers of the GP energy

$$
e(a):=\inf _{\left\{u \in \mathcal{H},\|u\|_{2}^{2}=1\right\}} E_{a}(u),
$$

where the space $\mathcal{H}$ is defined by

$$
\mathcal{H}:=\left\{u \in H^{1}\left(\mathbb{R}^{2}\right): \int_{\mathbb{R}^{2}} V(x)|u(x)|^{2} d x<\infty\right\} .
$$

The minimization problem $e(a)$ was analyzed recently in $[2,10,11,12,26$, and references therein. Existing results show that $e(a)$ is an $L^{2}$-critical constraint variational problem. Actually, it was shown in [2, 10] that $e(a)$ admits minimizers if and only if $a<a^{*}:=\|w\|_{2}^{2}$, where $w=w(|x|)$ is the unique (up to translations) radial positive solution (cf. [7, 19, 14]) of the following nonlinear scalar field equation

$$
\Delta w-w+w^{3}=0 \text { in } \mathbb{R}^{2} \text {, where } w \in H^{1}\left(\mathbb{R}^{2}\right) .
$$

It turns out that the existence and nonexistence of minimizers for $e(a)$ are well connected with the following Gagliardo-Nirenberg inequality

$$
\int_{\mathbb{R}^{2}}|u(x)|^{4} d x \leq \frac{2}{\|w\|_{2}^{2}} \int_{\mathbb{R}^{2}}|\nabla u(x)|^{2} d x \int_{\mathbb{R}^{2}}|u(x)|^{2} d x, \quad \forall u \in H^{1}\left(\mathbb{R}^{2}\right),
$$

where the equality is attained at $w$ (cf. [25]).

Since $E_{a}(u) \geq E_{a}(|u|)$ for any $u \in \mathcal{H}$, any minimizer $u_{a}$ of $e(a)$ must be either non-negative or non-positive, and it satisfies the Euler-Lagrange equation

$$
-\Delta u_{a}+V(x) u_{a}=\mu_{a} u_{a}+a u_{a}^{3} \quad \text { in } \mathbb{R}^{2},
$$

where $\mu_{a} \in \mathbb{R}$ is a suitable Lagrange multiplier. Thus, by applying the maximum principle to the equation (1.6), any minimizer $u_{a}$ of $e(a)$ is further either negative or positive. Therefore, without loss of generality one can restrict the minimizations of $e(a)$ to positive functions. In this paper positive minimizers of e $(a)$ are called ground states of attractive $B E C$. Applying energy estimates and blow-up analysis, the spike profiles of positive minimizers for $e(a)$ as $a \nearrow a^{*}$ were recently discussed in [10, 11, 12] under different types of potentials $V(x)$, see our Proposition 2.1 for some related results. In spite of these facts, it remains open to discuss the refined spike profiles of positive minimizers. On the other hand, the local uniqueness of positive minimizers for $e(a)$ as a.e. a $\nearrow a^{*}$ was also proved [11] by the ODE argument, for the case where $V(r)=V(|x|)$ is radially symmetric and satisfies $V^{\prime}(r) \geq 0$, see Corollary 1.1 in [11] for details. Here the locality of uniqueness means that $a$ is near $a^{*}$. It is therefore natural to ask whether such local uniqueness still holds for the case where $V(x)$ is not radially symmetric. We should remark that all these results mentioned above were obtained mainly by analyzing the variational structures of the minimization problem $e(a)$, instead of discussing the PDE properties of the associated elliptic equation (1.6).

By investigating thoroughly the associated equation (1.6), the main purpose of this paper is to derive the refined spike profiles of positive minimizers for $e(a)$ as $a \nearrow a^{*}$, and extend the above local uniqueness to the cases of non-symmetric potentials $V(x)$ as well. Throughout the whole paper, we shall consider the trapping potential $V(x)$ satisfying $\lim _{|x| \rightarrow \infty} V(x)=\infty$ in the class of homogeneous functions, for which we define 
Definition 1.1. $h(x) \geq 0$ in $\mathbb{R}^{2}$ is homogeneous of degree $p \in \mathbb{R}^{+}$(about the origin), if there exists some $p>0$ such that

$$
h(t x)=t^{p} h(x) \text { in } \mathbb{R}^{2} \text { for any } t>0 .
$$

Following [9, Remark 3.2], the above definition implies that the homogeneous function $h(x) \in C\left(\mathbb{R}^{2}\right)$ of degree $p>0$ satisfies

$$
0 \leq h(x) \leq C|x|^{p} \text { in } \mathbb{R}^{2},
$$

where $C>0$ denotes the maximum of $h(x)$ on $\partial B_{1}(0)$. Moreover, since we assume that $\lim _{|x| \rightarrow \infty} h(x)=\infty, x=0$ is the unique minimum point of $h(x)$. Additionally, we often need to assume that $V(x)=h(x) \in C^{2}\left(\mathbb{R}^{2}\right)$ satisfies

$$
y_{0} \text { is the unique critical point of } H(y)=\int_{\mathbb{R}^{2}} h(x+y) w^{2}(x) d x \text {. }
$$

The following example shows that for some non-symmetric potentials $h(x), H(y)$ admits a unique critical point $y_{0}$, where $y_{0}$ satisfies $y_{0} \neq 0$ and is non-degenerate in the sense that

$$
\operatorname{det}\left(\frac{\partial^{2} H\left(y_{0}\right)}{\partial x_{i} \partial x_{j}}\right) \neq 0, \text { where } i, j=1,2 \text {. }
$$

Example 1.1. Suppose that the potential $h(x)$ satisfies

$$
h(x)=|x|^{p}\left[1+\delta h_{0}(\theta)\right] \geq 0, \text { where } p \geq 2 \text { and } \delta \in \mathbb{R},
$$

where $h_{0}(\theta) \in C^{2}([0,2 \pi])$ satisfies

$$
\left(\int_{0}^{2 \pi} h_{0}(\theta) \cos \theta d \theta\right)^{2}+\left(\int_{0}^{2 \pi} h_{0}(\theta) \sin \theta d \theta\right)^{2}>0
$$

One can check from (1.12) that if $|\delta| \geq 0$ is small enough, then $H(y)$ admits a unique critical point $y_{0}=-\delta \hat{y}_{0} \in \mathbb{R}^{2}$, where $\hat{y}_{0}$ satisfies

$$
\hat{y}_{0} \sim\left(C_{1} \int_{0}^{2 \pi} h_{0}(\theta) \cos \theta d \theta, C_{2} \int_{0}^{2 \pi} h_{0}(\theta) \sin \theta d \theta\right) \neq(0,0) \text { as } \delta \rightarrow 0
$$

for some positive constants $C_{1}$ and $C_{2}$ depending only on $w$ and $p$. Furthermore, if $|\delta| \geq 0$ is small enough, then $\operatorname{det}\left(\frac{\partial^{2} H\left(y_{0}\right)}{\partial x_{i} \partial x_{j}}\right)>0$, which implies that the unique critical point $y_{0}$ of $H(y)$ is non-degenerate.

Our first main result is concerned with the following local uniqueness as $a \nearrow a^{*}$, which holds for some non-symmetric homogeneous potentials $h(x)$ in view of Example 1.1 .

Theorem 1.1. Suppose $V(x)=h(x) \in C^{2}\left(\mathbb{R}^{2}\right)$ is homogeneous of degree $p \geq 2$, where $\lim _{|x| \rightarrow \infty} h(x)=\infty$, and satisfies

$y_{0}$ is the unique and non-degenerate critical point of $H(y)=\int_{\mathbb{R}^{2}} h(x+y) w^{2}(x) d x$.

Then there exists a unique positive minimizer for $e(a)$ as a $\nearrow a^{*}$. 
The local uniqueness of Theorem 1.1 means that positive minimizers of $e(a)$ must be unique as $a$ is near $a^{*}$. It is possible to extend Theorem 1.1 to more general potentials $V(x)=g(x) h(x)$ for a class of functions $g(x)$, which is however beyond the discussion ranges of the present paper. We also remark that the proof of Theorem 1.1 is more involved for the case where $y_{0} \neq 0$ occurs in (1.14). Our proof of such local uniqueness is motivated by [3, 6, 9. Roughly speaking, as derived in Proposition 2.1 we shall first obtain some fundamental estimates on the spike behavior of positive minimizers. Under the non-degeneracy assumption of (1.14), the local uniqueness is then proved in Subsection 2.1 by establishing various types of local Pohozaev identities.

The proof of Theorem 1.1 shows that if one considers the local uniqueness of Theorem 1.1 in other dimensional cases, where $\mathbb{R}^{2}$ is replaced by $\mathbb{R}^{d}$ and $u^{4}$ is replaced by $u^{2+\frac{4}{d}}$ for $d \neq 2$, the fundamental estimates of Proposition 2.1 are not enough. Therefore, in the following we address the refined spike behavior of positive minimizers under the assumption (1.14). To introduce our second main result, for convenience we next denote

$$
\lambda_{0}=\left(\frac{p}{2} \int_{\mathbb{R}^{2}} h\left(x+y_{0}\right) w^{2}(x) d x\right)^{\frac{1}{2+p}}
$$

where $y_{0} \in \mathbb{R}^{2}$ is given by (1.14), and

$$
\psi(x)=\varphi(x)-\frac{C^{*}}{2}[w(x)+x \cdot \nabla w(x)],
$$

where $\varphi(x) \in C^{2}\left(\mathbb{R}^{2}\right) \cap L^{\infty}\left(\mathbb{R}^{2}\right)$ is the unique solution of

$$
\nabla \varphi(0)=0 \text { and }\left[-\Delta+\left(1-3 w^{2}\right)\right] \varphi(x)=-\frac{2 w^{3}}{\int_{\mathbb{R}^{2}} w^{4}}-\frac{2 h\left(x+y_{0}\right) w}{p \int_{\mathbb{R}^{2}} h\left(x+y_{0}\right) w^{2}} \text { in } \mathbb{R}^{2},
$$

and the nonzero constant $C^{*}$ is given by

$$
C^{*}=\frac{2}{2+p}\left(2 \int_{\mathbb{R}^{2}} w \psi_{3}+\int_{\mathbb{R}^{2}} \varphi^{2}\right)
$$

with $\psi_{3} \in C^{2}\left(\mathbb{R}^{2}\right) \cap L^{\infty}\left(\mathbb{R}^{2}\right)$ being the unique solution of (3.29). Using above notations, we shall derive the following theorem.

Theorem 1.2. Suppose $V(x)=h(x) \in C^{2}\left(\mathbb{R}^{2}\right)$ is homogeneous of degree $p \geq 2$, where $\lim _{|x| \rightarrow \infty} h(x)=\infty$, and satisfies (1.14) for some $y_{0} \in \mathbb{R}^{2}$. If $u_{a}$ is a positive minimizer of $e(a)$ as a $\nearrow a^{*}$, then we have

$$
\begin{aligned}
u_{a}(x)= & \frac{\lambda_{0}}{\|w\|_{2}}\left\{\frac{1}{\left(a^{*}-a\right)^{\frac{1}{2+p}}} w\left(\frac{\lambda_{0}\left(x-x_{a}\right)}{\left(a^{*}-a\right)^{\frac{1}{2+p}}}\right)+\left(a^{*}-a\right)^{\frac{1+p}{2+p}} \psi\left(\frac{\lambda_{0}\left(x-x_{a}\right)}{\left(a^{*}-a\right)^{\frac{1}{2+p}}}\right)\right. \\
& \left.+\left(a^{*}-a\right)^{\frac{3+2 p}{2+p}} \phi_{0}\left(\frac{\lambda_{0}\left(x-x_{a}\right)}{\left(a^{*}-a\right)^{\frac{1}{2+p}}}\right)\right\}+o\left(\left(a^{*}-a\right)^{\frac{3+2 p}{2+p}}\right) \text { as a } \nearrow a^{*}
\end{aligned}
$$

uniformly in $\mathbb{R}^{2}$ for some function $\phi_{0} \in C^{2}\left(\mathbb{R}^{2}\right) \cap L^{\infty}\left(\mathbb{R}^{2}\right)$, where $x_{a}$ is the unique maximum point of $u_{a}$ satisfying

$$
\left|\frac{\lambda x_{a}}{\left(a^{*}-a\right)^{\frac{1}{2+p}}}-y_{0}\right|=\left(a^{*}-a\right) O\left(\left|y^{0}\right|\right) \text { as } \text { a } \nearrow a^{*}
$$

for some $y^{0} \in \mathbb{R}^{2}$. 
Theorem 1.2 is derived directly from Theorem 1.1 and Theorem 3.6 in Section 3 with more details, where $\phi_{0} \in C^{2}\left(\mathbb{R}^{2}\right) \cap L^{\infty}\left(\mathbb{R}^{2}\right)$ is given explicitly. In Section 4 we shall extend the refined spike behavior of Theorem 1.2 to more general potentials $V(x)=g(x) h(x)$, where $h(-x)=h(x)$ is homogeneous and satisfies (1.14) and $0 \leq C \leq g(x) \leq \frac{1}{C}$ holds in $\mathbb{R}^{2}$, see Theorem 4.4 for details. To establish Theorem 1.2 and Theorem 4.4 , our Proposition 2.1 shows that the arguments of [10, 11, 12] give the leading expansion terms of the minimizer $u_{a}$ and the associated Lagrange multiplier $\mu_{a}$ satisfying (1.6) as well. In order to get (1.17) for the rest terms of $u_{a}$, the difficulty is to obtain the more precise estimate of $\mu_{a}$, which is overcome by the very delicate analysis of the associated equation (1.6), together with the constraint condition of $u_{a}$.

This paper is organized as follows: In Section 2 we shall prove Theorem 1.1 on the local uniqueness of positive minimizers. Section 3 is concerned with proving Theorem 1.2 on the refined spike profiles of positive minimizers for $e(a)$ as $a \nearrow a^{*}$. The main aim of Section 4 is to derive Theorem 4.4, which extends the refined spike behavior of Theorem 1.2 to more general potentials $V(x)=g(x) h(x)$. We shall leave the proof of Lemma 3.4 to Appendix A.

\section{Local Uniqueness of Positive Minimizers}

This section is devoted to the proof of Theorem 1.1 on the local uniqueness of positive minimizers. Towards this purpose, we need some estimates of positive minimizers for $e(a)$ as $a \nearrow a^{*}$, which hold essentially for more general potential $V(x) \in C^{2}\left(\mathbb{R}^{2}\right)$ satisfying

$V(x)=g(x) h(x)$, where $0<C \leq g(x) \leq \frac{1}{C}$ in $\mathbb{R}^{2}$ and $h(x)$ is homogeneous of

degree $p \geq 2$.

For convenience, we always denote $\left\{u_{k}\right\}$ to be a positive minimizer sequence of $e\left(a_{k}\right)$ with $a_{k} \nearrow a^{*}$ as $k \rightarrow \infty$, and define

$$
\lambda=\left(\frac{p g(0)}{2} \int_{\mathbb{R}^{2}} h\left(x+y_{0}\right) w^{2}(x) d x\right)^{\frac{1}{2+p}},
$$

where $V(x)=g(x) h(x)$ is assumed to satisfy (2.1) with $p \geq 2$ and $y_{0} \in \mathbb{R}^{2}$ is given by (1.9). Recall from (1.4) that $w(|x|)$ satisfies

$$
\int_{\mathbb{R}^{2}}|\nabla w|^{2} d x=\int_{\mathbb{R}^{2}}|w|^{2} d x=\frac{1}{2} \int_{\mathbb{R}^{2}}|w|^{4} d x
$$

see also Lemma 8.1.2 in [4]. Moreover, it follows from [7, Prop. 4.1] that $w$ admits the following exponential decay

$$
w(x),|\nabla w(x)|=O\left(|x|^{-\frac{1}{2}} e^{-|x|}\right) \quad \text { as }|x| \rightarrow \infty .
$$

Proposition 2.1. Suppose $V(x)=g(x) h(x) \in C^{2}\left(\mathbb{R}^{2}\right)$ satisfies $\lim _{|x| \rightarrow \infty} V(x)=\infty$ and (2.1), and assume (1.9) holds for some $y_{0} \in \mathbb{R}^{2}$. Then there exist a subsequence, still denoted by $\left\{a_{k}\right\}$, of $\left\{a_{k}\right\}$ and $\left\{x_{k}\right\} \subset \mathbb{R}^{2}$ such that

(I). The subsequence $\left\{u_{k}\right\}$ satisfies

$$
\left(a^{*}-a_{k}\right)^{\frac{1}{2+p}} u_{k}\left(x_{k}+x\left(a^{*}-a_{k}\right)^{\frac{1}{2+p}}\right) \rightarrow \frac{\lambda w(\lambda x)}{\|w\|_{2}} \text { as } k \rightarrow \infty
$$


uniformly in $\mathbb{R}^{2}$, and $x_{k}$ is the unique maximum point of $u_{k}$ satisfying

$$
\lim _{k \rightarrow \infty} \frac{\lambda x_{k}}{\left(a^{*}-a_{k}\right)^{\frac{1}{2+p}}}=y_{0},
$$

where $y_{0} \in \mathbb{R}^{2}$ is the same as that of (1.9). Moreover, $u_{k}$ satisfies

$$
\left(a^{*}-a_{k}\right)^{\frac{1}{2+p}} u_{k}\left(x_{k}+x\left(a^{*}-a_{k}\right)^{\frac{1}{2+p}}\right) \leq C e^{-\frac{\lambda}{2}|x|} \text { in } \mathbb{R}^{2},
$$

where the constant $C>0$ is independent of $k$.

(II). The energy $e\left(a_{k}\right)$ satisfies

$$
\lim _{k \rightarrow \infty} \frac{e\left(a_{k}\right)}{\left(a^{*}-a_{k}\right)^{p /(2+p)}}=\frac{\lambda^{2}}{a^{*}} \frac{p+2}{p} .
$$

Proof. Since the proof of Proposition 2.1 is similar to those in [10, 11, 12, which handle (1.1) with different potentials $V(x)$, we shall briefly sketch the structure of the proof.

If $V(x) \in C^{2}\left(\mathbb{R}^{2}\right)$ satisfies (2.1) with $p \geq 2$, we note that $h(x) \geq 0$ satisfies (1.8). Take the test function

$$
u_{\tau}(x)=A_{\tau} \frac{\tau}{\|w\|_{2}} \varphi(x) w(\tau x),
$$

where the nonnegative cut-off function $\varphi \in C_{0}^{\infty}\left(\mathbb{R}^{2}\right)$ satisfies $0 \leq \varphi(x) \leq 1$ in $\mathbb{R}^{2}$, and $A_{\tau}>0$ is chosen so that $\int_{\mathbb{R}^{2}} u_{\tau}(x)^{2} d x=1$. The same proof of Lemma 3 in [10] then yields that

$$
e(a) \leq C\left(a^{*}-a\right)^{\frac{p}{p+2}} \text { for } 0 \leq a<a^{*},
$$

where the constant $C>0$ is independent of $a$. By (2.9), we can follow Lemma 4 in [10] to derive that there exists a positive constant $K$, independent of $a$, such that

$$
\int_{\mathbb{R}^{2}}\left|u_{a}(x)\right|^{4} d x \leq \frac{1}{K}\left(a^{*}-a\right)^{-\frac{2}{p+2}} \text { for } 0 \leq a<a^{*},
$$

where $u_{a}>0$ is any minimizer of $e(a)$. Applying (2.9) and (2.10), a proof similar to that of Theorem 2.1 in [12] then gives that there exist two positive constants $m<M$, independent of $a$, such that

$$
m\left(a^{*}-a\right)^{\frac{p}{p+2}} \leq e(a) \leq M\left(a^{*}-a\right)^{\frac{p}{p+2}} \text { for } 0 \leq a<a^{*} .
$$

Based on (2.11), similar to Theorems 1.2 and 1.3 in [12], one can further deduce that there exist a subsequence (still denoted by $\left\{a_{k}\right\}$ ) of $\left\{a_{k}\right\}$ and $\left\{x_{k}\right\} \subset \mathbb{R}^{2}$, where $a_{k} \nearrow a^{*}$ as $k \rightarrow \infty$, such that (2.7) and (2.8) hold, and

$$
\left(a^{*}-a_{k}\right)^{\frac{1}{2+p}} u_{k}\left(x_{k}+x\left(a^{*}-a_{k}\right)^{\frac{1}{2+p}}\right) \rightarrow \frac{\lambda w(\lambda x)}{\|w\|_{2}} \text { strongly in } H^{1}\left(\mathbb{R}^{2}\right)
$$

as $k \rightarrow \infty$, where $x_{k}$ is the unique maximum point of $u_{k}$. Finally, since $w$ decays exponentially, the standard elliptic regularity theory applied to (2.12) yields that (2.5) holds uniformly in $\mathbb{R}^{2}$ (e.g. Lemma 4.9 in [18] for similar arguments).

We finally follow (1.9) and (2.5) to derive the estimate (2.6). Following (2.5), we define

$$
\bar{u}_{k}(x):=\frac{\sqrt{a^{*}} \varepsilon_{k}}{\lambda} u_{k}\left(\frac{\varepsilon_{k}}{\lambda} x+x_{k}\right), \text { where } \varepsilon_{k}:=\left(a^{*}-a_{k}\right)^{\frac{1}{2+p}}>0
$$


so that $\bar{u}_{k}(x) \rightarrow w(x)$ uniformly in $\mathbb{R}^{2}$ as $k \rightarrow \infty$. We then derive from (1.5) that

$$
\begin{aligned}
e\left(a_{k}\right)=E_{a_{k}}\left(u_{k}\right) & =\frac{\lambda^{2}}{a^{*} \varepsilon_{k}^{2}}\left[\int_{\mathbb{R}^{2}}\left|\nabla \bar{u}_{k}(x)\right|^{2} d x-\frac{1}{2} \int_{\mathbb{R}^{2}} \bar{u}_{k}^{4}(x) d x\right]+\frac{\lambda^{2} \varepsilon_{k}^{p}}{2\left(a^{*}\right)^{2}} \int_{\mathbb{R}^{2}} \bar{u}_{k}^{4}(x) d x \\
& +\frac{1}{a^{*}} \int_{\mathbb{R}^{2}} V\left(\frac{\varepsilon_{k}}{\lambda} x+x_{k}\right) \bar{u}_{k}^{2}(x) d x \\
& \geq \frac{\lambda^{2} \varepsilon_{k}^{p}}{2\left(a^{*}\right)^{2}} \int_{\mathbb{R}^{2}} \bar{u}_{k}^{4}(x) d x+\frac{1}{a^{*}}\left(\frac{\varepsilon_{k}}{\lambda}\right)^{p} \int_{\mathbb{R}^{2}} g\left(\frac{\varepsilon_{k}}{\lambda} x+x_{k}\right) h\left(x+\frac{\lambda x_{k}}{\varepsilon_{k}}\right) \bar{u}_{k}^{2}(x) d x,
\end{aligned}
$$

which then implies from (2.5) that $\left|\frac{\lambda x_{k}}{\varepsilon_{k}}\right|$ is bounded uniformly in $k$. Therefore, there exist a subsequence (still denoted by $\left\{\frac{\lambda x_{k}}{\varepsilon_{k}}\right\}$ ) of $\left\{\frac{\lambda x_{k}}{\varepsilon_{k}}\right\}$ and $y^{0} \in \mathbb{R}^{2}$ such that

$$
\frac{\lambda x_{k}}{\varepsilon_{k}} \rightarrow y^{0} \text { as } k \rightarrow \infty
$$

Note that

$$
\begin{aligned}
& \liminf _{k \rightarrow \infty} \int_{\mathbb{R}^{2}} g\left(\frac{\varepsilon_{k}}{\lambda} x+x_{k}\right) h\left(x+\frac{\lambda x_{k}}{\varepsilon_{k}}\right) \bar{u}_{k}^{2}(x) d x \\
\geq & \liminf _{k \rightarrow \infty} \int_{B} \frac{1}{\sqrt{\varepsilon_{k}}}(0) \\
= & g(0) \int_{\mathbb{R}^{2}} h\left(x+y^{0}\right) w^{2}(x) d x .
\end{aligned}
$$

Since $u_{k}$ gives the least energy of $e\left(a_{k}\right)$ and the assumption (1.9) implies that $y_{0}$ is essentially the unique global minimum point of $H(y)=\int_{\mathbb{R}^{2}} h(x+y) w^{2}(x) d x$, we conclude from (2.13) and (2.14) that $y^{0}=y_{0}$, which thus implies that (2.6) holds, and the proof is therefore complete.

\subsection{Proof of local uniqueness}

Following Proposition 2.1, this subsection is focussed on the proof of Theorem 1.1, and in the whole subsection we always assume that $V(x)=h(x) \in C^{2}\left(\mathbb{R}^{2}\right)$ is homogeneous of degree $p \geq 2$ and satisfies (1.14) and $\lim _{|x| \rightarrow \infty} h(x)=\infty$. Our proof is stimulated by [3, 6, 9]. We first define the linearized operator $\mathcal{L}$ by

$$
\mathcal{L}:=-\Delta+\left(1-3 w^{2}\right) \text { in } \mathbb{R}^{2},
$$

where $w=w(|x|)>0$ is the unique positive solution of (1.4) and $w$ satisfies the exponential decay (2.4). Recall from [14, 20] that

$$
\operatorname{ker}(\mathcal{L})=\operatorname{span}\left\{\frac{\partial w}{\partial x_{1}}, \frac{\partial w}{\partial x_{2}}\right\} .
$$

For any positive minimizer $u_{k}$ of $e\left(a_{k}\right)$, where $a_{k} \nearrow a^{*}$ as $k \rightarrow \infty$, one can note that $u_{k}$ solves the Euler-Lagrange equation

$$
-\Delta u_{k}(x)+V(x) u_{k}(x)=\mu_{k} u_{k}(x)+a_{k} u_{k}^{3}(x) \text { in } \mathbb{R}^{2},
$$

where $\mu_{k} \in \mathbb{R}$ is a suitable Lagrange multiplier and satisfies

$$
\mu_{k}=e\left(a_{k}\right)-\frac{a_{k}}{2} \int_{\mathbb{R}^{2}} u_{k}^{4}(x) d x
$$


Moreover, under the more general assumption (2.1), one can derive from (2.3) and (2.5) that $u_{k}$ satisfies

$$
\int_{\mathbb{R}^{2}} u_{k}^{4}(x) d x=\left(a^{*}-a_{k}\right)^{-\frac{2}{2+p}}\left[\frac{2 \lambda^{2}}{a^{*}}+o(1)\right] \text { as } k \rightarrow \infty .
$$

It then follows from (2.3), (2.17) and (2.18) that $\mu_{k}$ satisfies

$$
\frac{\mu_{k} \varepsilon_{k}^{2}}{\lambda^{2}} \rightarrow-1 \text { as } k \rightarrow+\infty
$$

where we denote

$$
\varepsilon_{k}:=\left(a^{*}-a_{k}\right)^{\frac{1}{2+p}}>0 .
$$

Set

$$
\bar{u}_{k}(x):=\frac{\sqrt{a^{*}} \varepsilon_{k}}{\lambda} u_{k}\left(\frac{\varepsilon_{k}}{\lambda} x+x_{k}\right),
$$

so that Proposition 2.1 gives $\bar{u}_{k}(x) \rightarrow w(x)$ uniformly in $\mathbb{R}^{2}$ as $k \rightarrow \infty$. Note from (2.16) that $\bar{u}_{k}$ satisfies

$$
-\Delta \bar{u}_{k}(x)+\left(\frac{\varepsilon_{k}}{\lambda}\right)^{2} V\left(\frac{\varepsilon_{k}}{\lambda} x+x_{k}\right) \bar{u}_{k}(x)=\frac{\mu_{k} \varepsilon_{k}^{2}}{\lambda^{2}} \bar{u}_{k}(x)+\frac{a_{k}}{a^{*}} \bar{u}_{k}^{3}(x) \text { in } \mathbb{R}^{2} .
$$

Moreover, by the exponential decay (2.7), there exist $C_{0}>0$ and $R>0$ such that

$$
\left|\bar{u}_{k}(x)\right| \leq C_{0} e^{-\frac{|x|}{2}} \text { for }|x|>R,
$$

which then implies that

$$
\left|\left(\frac{\varepsilon_{k}}{\lambda}\right)^{2} V\left(\frac{\varepsilon_{k}}{\lambda} x+x_{k}\right) \bar{u}_{k}(x)\right| \leq C C_{0} e^{-\frac{|x|}{4}} \text { for }|x|>R,
$$

if $V(x)$ satisfies (2.1) with $p \geq 2$. Therefore, under the assumption (2.1), applying the local elliptic estimates (see (3.15) in [8]) to (2.20) yields that

$$
\left|\nabla \bar{u}_{k}(x)\right| \leq C e^{-\frac{|x|}{4}} \text { as }|x| \rightarrow \infty
$$

where the estimates (2.19) and (2.21) are also used. In the following, we shall follow Proposition 2.1 and (2.22) to derive Theorem 1.1 on the local uniqueness of positive minimizers as $a \nearrow a^{*}$.

Proof of Theorem 1.1. Suppose that there exist two different positive minimizers $u_{1, k}$ and $u_{2, k}$ of $e\left(a_{k}\right)$ with $a_{k} \nearrow a^{*}$ as $k \rightarrow \infty$. Let $x_{1, k}$ and $x_{2, k}$ be the unique local maximum point of $u_{1, k}$ and $u_{2, k}$, respectively. Following (2.16), $u_{i, k}$ then solves the Euler-Lagrange equation

$$
-\Delta u_{i, k}(x)+h(x) u_{i, k}(x)=\mu_{i, k} u_{i, k}(x)+a_{k} u_{i, k}^{3}(x) \quad \text { in } \mathbb{R}^{2}, \quad i=1,2,
$$

where $V(x)=h(x)$ and $\mu_{i, k} \in \mathbb{R}$ is a suitable Lagrange multiplier. Define

$$
\bar{u}_{i, k}(x):=\frac{\sqrt{a^{*}} \varepsilon_{k}}{\lambda} u_{i, k}(x), \text { where } i=1,2 .
$$


Proposition 2.1 then implies that $\bar{u}_{i, k}\left(\frac{\varepsilon_{k}}{\lambda} x+x_{2, k}\right) \rightarrow w(x)$ uniformly in $\mathbb{R}^{2}$, and $\bar{u}_{i, k}$ satisfies the equation

$$
-\varepsilon_{k}^{2} \Delta \bar{u}_{i, k}(x)+\varepsilon_{k}^{2} h(x) \bar{u}_{i, k}(x)=\mu_{i, k} \varepsilon_{k}^{2} \bar{u}_{i, k}(x)+\frac{\lambda^{2} a_{k}}{a^{*}} \bar{u}_{i, k}^{3}(x) \quad \text { in } \mathbb{R}^{2}, \quad i=1,2 .
$$

Because $u_{1, k} \not \equiv u_{2, k}$, we consider

$$
\bar{\xi}_{k}(x)=\frac{u_{2, k}(x)-u_{1, k}(x)}{\left\|u_{2, k}-u_{1, k}\right\|_{L^{\infty}\left(\mathbb{R}^{2}\right)}}=\frac{\bar{u}_{2, k}(x)-\bar{u}_{1, k}(x)}{\left\|\bar{u}_{2, k}-\bar{u}_{1, k}\right\|_{L^{\infty}\left(\mathbb{R}^{2}\right)}} .
$$

Then $\bar{\xi}_{k}$ satisfies the equation

$$
-\varepsilon_{k}^{2} \Delta \bar{\xi}_{k}+\bar{C}_{k}(x) \bar{\xi}_{k}=\bar{g}_{k}(x) \text { in } \mathbb{R}^{2},
$$

where the coefficient $\bar{C}_{k}(x)$ satisfies

$$
\bar{C}_{k}(x):=-\mu_{1, k} \varepsilon_{k}^{2}-\frac{\lambda^{2} a_{k}}{a^{*}}\left(\bar{u}_{2, k}^{2}+\bar{u}_{2, k} \bar{u}_{1, k}+\bar{u}_{1, k}^{2}\right)+\varepsilon_{k}^{2} h(x),
$$

and the nonhomogeneous term $\bar{g}_{k}(x)$ satisfies

$$
\begin{aligned}
\bar{g}_{k}(x):=\frac{\varepsilon_{k}^{2} \bar{u}_{2, k}\left(\mu_{2, k}-\mu_{1, k}\right)}{\left\|\bar{u}_{2, k}-\bar{u}_{1, k}\right\|_{L^{\infty}\left(\mathbb{R}^{2}\right)}} & =-\frac{\lambda^{4} a_{k} \bar{u}_{2, k}}{2\left(a^{*}\right)^{2} \varepsilon_{k}^{2}} \int_{\mathbb{R}^{2}} \frac{\bar{u}_{2, k}^{4}-\bar{u}_{1, k}^{4}}{\left\|\bar{u}_{2, k}-\bar{u}_{1, k}\right\|_{L^{\infty}\left(\mathbb{R}^{2}\right)}} d x \\
& =-\frac{\lambda^{4} a_{k} \bar{u}_{2, k}}{2\left(a^{*}\right)^{2} \varepsilon_{k}^{2}} \int_{\mathbb{R}^{2}} \bar{\xi}_{k}\left(\bar{u}_{2, k}^{2}+\bar{u}_{1, k}^{2}\right)\left(\bar{u}_{2, k}+\bar{u}_{1, k}\right) d x,
\end{aligned}
$$

due to the relation (2.17).

Motivated by [3], we first claim that for any $x_{0} \in \mathbb{R}^{2}$, there exists a small constant $\delta>0$ such that

$$
\int_{\partial B_{\delta}\left(x_{0}\right)}\left[\varepsilon_{k}^{2}\left|\nabla \bar{\xi}_{k}\right|^{2}+\frac{\lambda^{2}}{2}\left|\bar{\xi}_{k}\right|^{2}+\varepsilon_{k}^{2} h(x)\left|\bar{\xi}_{k}\right|^{2}\right] d S=O\left(\varepsilon_{k}^{2}\right) \quad \text { as } k \rightarrow \infty .
$$

To prove the above claim, multiplying (2.26) by $\bar{\xi}_{k}$ and integrating over $\mathbb{R}^{2}$, we obtain that

$$
\begin{aligned}
& \varepsilon_{k}^{2} \int_{\mathbb{R}^{2}}\left|\nabla \bar{\xi}_{k}\right|^{2}-\mu_{i, k} \varepsilon_{k}^{2} \int_{\mathbb{R}^{2}}\left|\bar{\xi}_{k}\right|^{2}+\varepsilon_{k}^{2} \int_{\mathbb{R}^{2}} h(x)\left|\bar{\xi}_{k}\right|^{2} \\
= & \frac{\lambda^{2} a_{k}}{a^{*}} \int_{\mathbb{R}^{2}}\left(\bar{u}_{2, k}^{2}+\bar{u}_{2, k} \bar{u}_{1, k}+\bar{u}_{1, k}^{2}\right)\left|\bar{\xi}_{k}\right|^{2} \\
& -\frac{\lambda^{4} a_{k}}{2\left(a^{*}\right)^{2} \varepsilon_{k}^{2}} \int_{\mathbb{R}^{2}} \bar{u}_{2, k} \bar{\xi}_{k} \int_{\mathbb{R}^{2}} \bar{\xi}_{k}\left(\bar{u}_{2, k}^{2}+\bar{u}_{1, k}^{2}\right)\left(\bar{u}_{2, k}+\bar{u}_{1, k}\right) \\
\leq & \frac{\lambda^{2} a_{k}}{a^{*}} \int_{\mathbb{R}^{2}}\left(\bar{u}_{2, k}^{2}+\bar{u}_{2, k} \bar{u}_{1, k}+\bar{u}_{1, k}^{2}\right)+\frac{\lambda^{4} a_{k}}{2\left(a^{*}\right)^{2} \varepsilon_{k}^{2}} \int_{\mathbb{R}^{2}} \bar{u}_{2, k} \int_{\mathbb{R}^{2}}\left(\bar{u}_{2, k}^{2}+\bar{u}_{1, k}^{2}\right)\left(\bar{u}_{2, k}+\bar{u}_{1, k}\right) \\
\leq & C \varepsilon_{k}^{2} \text { as } k \rightarrow \infty,
\end{aligned}
$$

since $\left|\bar{\xi}_{k}\right|$ and $\bar{u}_{i, k}\left(\frac{\varepsilon_{k}}{\lambda} x+x_{2, k}\right)$ are bounded uniformly in $k$, and $\bar{u}_{i, k}\left(\frac{\varepsilon_{k}}{\lambda} x+x_{2, k}\right)$ decays exponentially as $|x| \rightarrow \infty, i=1,2$. This implies that there exists a constant $C_{1}>0$ such that

$$
I:=\varepsilon_{k}^{2} \int_{\mathbb{R}^{2}}\left|\nabla \bar{\xi}_{k}\right|^{2}+\frac{\lambda^{2}}{2} \int_{\mathbb{R}^{2}}\left|\bar{\xi}_{k}\right|^{2}+\varepsilon_{k}^{2} \int_{\mathbb{R}^{2}} h(x)\left|\bar{\xi}_{k}\right|^{2}<C_{1} \varepsilon_{k}^{2} \quad \text { as } k \rightarrow \infty
$$


Applying Lemma 4.5 in [3], we then conclude that for any $x_{0} \in \mathbb{R}^{2}$, there exist a small constant $\delta>0$ and $C_{2}>0$ such that

$$
\int_{\partial B_{\delta}\left(x_{0}\right)}\left[\varepsilon_{k}^{2}\left|\nabla \bar{\xi}_{k}\right|^{2}+\frac{\lambda^{2}}{2}\left|\bar{\xi}_{k}\right|^{2}+\varepsilon_{k}^{2} h(x)\left|\bar{\xi}_{k}\right|^{2}\right] d S \leq C_{2} I \leq C_{1} C_{2} \varepsilon_{k}^{2} \text { as } k \rightarrow \infty,
$$

which therefore implies the claim (2.29).

We next define

$$
\xi_{k}(x)=\bar{\xi}_{k}\left(\frac{\varepsilon_{k}}{\lambda} x+x_{2, k}\right), \quad k=1,2, \cdots,
$$

and

$$
\tilde{u}_{i, k}(x):=\frac{\sqrt{a^{*}} \varepsilon_{k}}{\lambda} u_{i, k}\left(\frac{\varepsilon_{k}}{\lambda} x+x_{2, k}\right), \text { where } i=1,2,
$$

so that $\tilde{u}_{i, k}(x) \rightarrow w(x)$ uniformly in $\mathbb{R}^{2}$ as $k \rightarrow \infty$ in view of Proposition [2.1. Under the non-degeneracy assumption (1.14), we shall carry out the proof of Theorem 1.1 by deriving a contradiction through the following three steps.

Step 1. There exist a subsequence $\left\{a_{k}\right\}$ and some constants $b_{0}, b_{1}$ and $b_{2}$ such that $\xi_{k}(x) \rightarrow \xi_{0}(x)$ in $C_{l o c}\left(\mathbb{R}^{2}\right)$ as $k \rightarrow \infty$, where

$$
\xi_{0}(x)=b_{0}(w+x \cdot \nabla w)+\sum_{i=1}^{2} b_{i} \frac{\partial w}{\partial x_{i}} .
$$

Note that $\xi_{k}$ satisfies

$$
-\Delta \xi_{k}+C_{k}(x) \xi_{k}=g_{k}(x) \text { in } \mathbb{R}^{2}
$$

where the coefficient $C_{k}(x)$ satisfies

$$
\begin{aligned}
C_{k}(x):= & -\left(1-\frac{\varepsilon_{k}^{2+p}}{a^{*}}\right)\left[\tilde{u}_{2, k}^{2}(x)+\tilde{u}_{2, k}(x) \tilde{u}_{1, k}(x)+\tilde{u}_{1, k}^{2}(x)\right] \\
& -\frac{\varepsilon_{k}^{2}}{\lambda^{2}} \mu_{1, k}+\frac{\varepsilon_{k}^{2}}{\lambda^{2}} h\left(\frac{\varepsilon_{k} x}{\lambda}+x_{2, k}\right),
\end{aligned}
$$

and the nonhomogeneous term $g_{k}(x)$ satisfies

$$
\begin{aligned}
g_{k}(x):=\frac{\tilde{u}_{2, k}}{\lambda^{2}} \frac{\varepsilon_{k}^{2}\left(\mu_{2, k}-\mu_{1, k}\right)}{\left\|\tilde{u}_{2, k}-\tilde{u}_{1, k}\right\|_{L^{\infty}}} & =-\frac{\tilde{u}_{2, k}}{\lambda^{2}} \frac{a_{k} \varepsilon_{k}^{2}}{2} \int_{\mathbb{R}^{2}} \frac{u_{2, k}^{4}-u_{1, k}^{4}}{\left\|\tilde{u}_{2, k}-\tilde{u}_{1, k}\right\|_{L^{\infty}}} d x \\
& =-\frac{a_{k} \tilde{u}_{2, k}}{2\left(a^{*}\right)^{2}} \int_{\mathbb{R}^{2}} \xi_{k}\left(\tilde{u}_{2, k}^{2}+\tilde{u}_{1, k}^{2}\right)\left(\tilde{u}_{2, k}+\tilde{u}_{1, k}\right) d x .
\end{aligned}
$$

Here we have used (2.17) and (2.25). Since $\left\|\xi_{k}\right\|_{L^{\infty}\left(\mathbb{R}^{2}\right)} \leq 1$, the standard elliptic regularity then implies (cf. [8]) that $\left\|\xi_{k}\right\|_{C_{l o c}^{1, \alpha}\left(\mathbb{R}^{2}\right)} \leq C$ for some $\alpha \in(0,1)$, where the constant $C>0$ is independent of $k$. Therefore, there exist a subsequence $\left\{a_{k}\right\}$ and a function $\xi_{0}=\xi_{0}(x)$ such that $\xi_{k}(x) \rightarrow \xi_{0}(x)$ in $C_{l o c}\left(\mathbb{R}^{2}\right)$ as $k \rightarrow \infty$. Applying Proposition 2.1, direct calculations yield from (2.17) and (2.18) that

$$
C_{k}(x) \rightarrow 1-3 w^{2}(x) \text { uniformly on } \mathbb{R}^{2} \text { as } k \rightarrow \infty,
$$

and

$$
g_{k}(x) \rightarrow-\frac{2 w(x)}{a^{*}} \int_{\mathbb{R}^{2}} w^{3} \xi_{0} \text { uniformly on } \mathbb{R}^{2} \text { as } k \rightarrow \infty
$$


This implies from (2.33) that $\xi_{0}$ solves

$$
\mathcal{L} \xi_{0}=-\Delta \xi_{0}+\left(1-3 w^{2}\right) \xi_{0}=\left(-\frac{2}{a^{*}} \int_{\mathbb{R}^{2}} w^{3} \xi_{0}\right) w \text { in } \mathbb{R}^{2} .
$$

Since $\mathcal{L}(w+x \cdot \nabla w)=-2 w$, we then conclude from (2.15) and (2.36) that (2.32) holds for some constants $b_{0}, b_{1}$ and $b_{2}$.

Step 2. The constants $b_{0}=b_{1}=b_{2}=0$ in (2.32).

We first derive the following Pohozaev-type identity

$$
b_{0} \int_{\mathbb{R}^{2}} \frac{\partial h\left(x+y_{0}\right)}{\partial x_{j}}\left(x \cdot \nabla w^{2}\right)-\sum_{i=1}^{2} b_{i} \int_{\mathbb{R}^{2}} \frac{\partial^{2} h\left(x+y_{0}\right)}{\partial x_{j} \partial x_{i}} w^{2}=0, \quad j=1,2 .
$$

Multiplying (2.25) by $\frac{\partial \bar{u}_{i, k}}{\partial x_{j}}$, where $i, j=1,2$, and integrating over $B_{\delta}\left(x_{2, k}\right)$, where $\delta>0$ is small and given by (2.29), we calculate that

$$
\begin{aligned}
& -\varepsilon_{k}^{2} \int_{B_{\delta}\left(x_{2, k}\right)} \frac{\partial \bar{u}_{i, k}}{\partial x_{j}} \Delta \bar{u}_{i, k}+\varepsilon_{k}^{2} \int_{B_{\delta}\left(x_{2, k}\right)} h(x) \frac{\partial \bar{u}_{i, k}}{\partial x_{j}} \bar{u}_{i, k} \\
= & \mu_{i, k} \varepsilon_{k}^{2} \int_{B_{\delta}\left(x_{2, k}\right)} \frac{\partial \bar{u}_{i, k}}{\partial x_{j}} \bar{u}_{i, k}+\frac{\lambda^{2} a_{k}}{a^{*}} \int_{B_{\delta}\left(x_{2, k}\right)} \frac{\partial \bar{u}_{i, k}}{\partial x_{j}} \bar{u}_{i, k}^{3} \\
= & \frac{1}{2} \mu_{i, k} \varepsilon_{k}^{2} \int_{\partial B_{\delta}\left(x_{2, k}\right)} \bar{u}_{i, k}^{2} \nu_{j} d S+\frac{\lambda^{2} a_{k}}{4 a^{*}} \int_{\partial B_{\delta}\left(x_{2, k}\right)} \bar{u}_{i, k}^{4} \nu_{j} d S,
\end{aligned}
$$

where $\nu=\left(\nu_{1}, \nu_{2}\right)$ denotes the outward unit normal of $\partial B_{\delta}\left(x_{2, k}\right)$. Note that

$$
\begin{aligned}
& -\varepsilon_{k}^{2} \int_{B_{\delta}\left(x_{2, k}\right)} \frac{\partial \bar{u}_{i, k}}{\partial x_{j}} \Delta \bar{u}_{i, k} \\
= & -\varepsilon_{k}^{2} \int_{\partial B_{\delta}\left(x_{2, k}\right)} \frac{\partial \bar{u}_{i, k}}{\partial x_{j}} \frac{\partial \bar{u}_{i, k}}{\partial \nu} d S+\varepsilon_{k}^{2} \int_{B_{\delta}\left(x_{2, k}\right)} \nabla \bar{u}_{i, k} \cdot \nabla \frac{\partial \bar{u}_{i, k}}{\partial x_{j}} \\
= & -\varepsilon_{k}^{2} \int_{\partial B_{\delta}\left(x_{2, k}\right)} \frac{\partial \bar{u}_{i, k}}{\partial x_{j}} \frac{\partial \bar{u}_{i, k}}{\partial \nu} d S+\frac{1}{2} \varepsilon_{k}^{2} \int_{\partial B_{\delta}\left(x_{2, k}\right)}\left|\nabla \bar{u}_{i, k}\right|^{2} \nu_{j} d S,
\end{aligned}
$$

and

$$
\varepsilon_{k}^{2} \int_{B_{\delta}\left(x_{2, k}\right)} h(x) \frac{\partial \bar{u}_{i, k}}{\partial x_{j}} \bar{u}_{i, k}=\frac{\varepsilon_{k}^{2}}{2} \int_{\partial B_{\delta}\left(x_{2, k}\right)} h(x) \bar{u}_{i, k}^{2} \nu_{j} d S-\frac{\varepsilon_{k}^{2}}{2} \int_{B_{\delta}\left(x_{2, k}\right)} \frac{\partial h(x)}{\partial x_{j}} \bar{u}_{i, k}^{2} .
$$

We then derive from (2.38) that

$$
\begin{aligned}
& \varepsilon_{k}^{2} \int_{B_{\delta}\left(x_{2, k}\right)} \frac{\partial h(x)}{\partial x_{j}} \bar{u}_{i, k}^{2} \\
= & -2 \varepsilon_{k}^{2} \int_{\partial B_{\delta}\left(x_{2, k}\right)} \frac{\partial \bar{u}_{i, k}}{\partial x_{j}} \frac{\partial \bar{u}_{i, k}}{\partial \nu} d S+\varepsilon_{k}^{2} \int_{\partial B_{\delta}\left(x_{2, k}\right)}\left|\nabla \bar{u}_{i, k}\right|^{2} \nu_{j} d S \\
& +\varepsilon_{k}^{2} \int_{\partial B_{\delta}\left(x_{2, k}\right)} h(x) \bar{u}_{i, k}^{2} \nu_{j} d S-\mu_{i, k} \varepsilon_{k}^{2} \int_{\partial B_{\delta}\left(x_{2, k}\right)} \bar{u}_{i, k}^{2} \nu_{j} d S \\
& -\frac{\lambda^{2} a_{k}}{2 a^{*}} \int_{\partial B_{\delta}\left(x_{2, k}\right)} \bar{u}_{i, k}^{4} \nu_{j} d S .
\end{aligned}
$$


Following (2.39), we thus have

$$
\begin{aligned}
& \varepsilon_{k}^{2} \int_{B_{\delta}\left(x_{2, k}\right)} \frac{\partial h(x)}{\partial x_{j}}\left(\bar{u}_{2, k}+\bar{u}_{1, k}\right) \bar{\xi}_{k} d x \\
= & -2 \varepsilon_{k}^{2} \int_{\partial B_{\delta}\left(x_{2, k}\right)}\left[\frac{\partial \bar{u}_{2, k}}{\partial x_{j}} \frac{\partial \bar{\xi}_{k}}{\partial \nu}+\frac{\partial \bar{\xi}_{k}}{\partial x_{j}} \frac{\partial \bar{u}_{1, k}}{\partial \nu}\right] d S \\
& +\varepsilon_{k}^{2} \int_{\partial B_{\delta}\left(x_{2, k}\right)} \nabla \bar{\xi}_{k} \cdot \nabla\left(\bar{u}_{2, k}+\bar{u}_{1, k}\right) \nu_{j} d S \\
& +\varepsilon_{k}^{2} \int_{\partial B_{\delta}\left(x_{2, k}\right)} h(x)\left(\bar{u}_{2, k}+\bar{u}_{1, k}\right) \bar{\xi}_{k} \nu_{j} d S-\mu_{1, k} \varepsilon_{k}^{2} \int_{\partial B_{\delta}\left(x_{2, k}\right)}\left(\bar{u}_{2, k}+\bar{u}_{1, k}\right) \bar{\xi}_{k} \nu_{j} d S \\
& -\frac{\lambda^{2} a_{k}}{2 a^{*}} \int_{\partial B_{\delta}\left(x_{2, k}\right)}\left(\bar{u}_{2, k}^{2}+\bar{u}_{1, k}^{2}\right)\left(\bar{u}_{2, k}+\bar{u}_{1, k}\right) \bar{\xi}_{k} \nu_{j} d S \\
& -\frac{\left(\mu_{2, k}-\mu_{1, k}\right) \varepsilon_{k}^{2}}{\left\|\bar{u}_{2, k}-\bar{u}_{1, k}\right\|_{L^{\infty}}} \int_{\partial B_{\delta}\left(x_{2, k}\right)} \bar{u}_{2, k}^{2} \nu_{j} d S .
\end{aligned}
$$

We now estimate the right hand side of (2.40) as follows. Applying (2.29), if $\delta>0$ is small, we then deduce that

$$
\begin{aligned}
& \varepsilon_{k}^{2} \int_{\partial B_{\delta}\left(x_{2, k}\right)}\left|\frac{\partial \bar{u}_{2, k}}{\partial x_{j}} \frac{\partial \bar{\xi}_{k}}{\partial \nu}\right| d S \\
\leq & \varepsilon_{k}\left(\int_{\partial B_{\delta}\left(x_{2, k}\right)}\left|\frac{\partial \bar{u}_{2, k}}{\partial x_{j}}\right|^{2} d S\right)^{\frac{1}{2}}\left(\varepsilon_{k}^{2} \int_{\partial B_{\delta}\left(x_{2, k}\right)}\left|\frac{\partial \bar{\xi}_{k}}{\partial \nu}\right|^{2} d S\right)^{\frac{1}{2}} \leq C \varepsilon_{k}^{2} e^{-\frac{C \delta}{\varepsilon_{k}}} \text { as } k \rightarrow \infty,
\end{aligned}
$$

due to the fact that $\nabla \bar{u}_{2, k}\left(\frac{\varepsilon_{k}}{\lambda} x+x_{2, k}\right)$ satisfies the exponential decay (2.22), where $C>0$ is independent of $k$. Similarly, we have

$$
\varepsilon_{k}^{2} \int_{\partial B_{\delta}\left(x_{2, k}\right)}\left|\frac{\partial \bar{\xi}_{k}}{\partial x_{j}} \frac{\partial \bar{u}_{1, k}}{\partial \nu}\right| d S \leq C \varepsilon_{k}^{2} e^{-\frac{C \delta}{\varepsilon_{k}}} \text { as } k \rightarrow \infty
$$

and

$$
\varepsilon_{k}^{2}\left|\int_{\partial B_{\delta}\left(x_{2, k}\right)} \nabla \bar{\xi}_{k} \cdot \nabla\left(\bar{u}_{2, k}+\bar{u}_{1, k}\right) \nu_{j} d S\right| \leq C \varepsilon_{k}^{2} e^{-\frac{C \delta}{\varepsilon_{k}}} \text { as } k \rightarrow \infty,
$$

On the other hand, since both $\left|\bar{\xi}_{k}\right|$ and $\left|\left(\mu_{2, k}-\mu_{1, k}\right) \varepsilon_{k}^{2}\right|$ are bounded uniformly in $k$, we also get from (2.22) that

$$
\begin{aligned}
& \mid \varepsilon_{k}^{2} \int_{\partial B_{\delta}\left(x_{2, k}\right)} h(x)\left(\bar{u}_{2, k}+\bar{u}_{1, k}\right) \bar{\xi}_{k} \nu_{j} d S-\mu_{1, k} \varepsilon_{k}^{2} \int_{\partial B_{\delta}\left(x_{2, k}\right)}\left(\bar{u}_{2, k}+\bar{u}_{1, k}\right) \bar{\xi}_{k} \nu_{j} d S \\
& -\frac{\lambda^{2} a_{k}}{2 a^{*}} \int_{\partial B_{\delta}\left(x_{2, k}\right)}\left(\bar{u}_{2, k}^{2}+\bar{u}_{1, k}^{2}\right)\left(\bar{u}_{2, k}+\bar{u}_{1, k}\right) \bar{\xi}_{k} \nu_{j} d S \\
& -\frac{\left(\mu_{2, k}-\mu_{1, k}\right) \varepsilon_{k}^{2}}{\left\|\bar{u}_{2, k}-\bar{u}_{1, k}\right\|_{L^{\infty}}} \int_{\partial B_{\delta}\left(x_{2, k}\right)} \bar{u}_{2, k}^{2} \nu_{j} d S \mid \\
= & o\left(e^{-\frac{C \delta}{\varepsilon_{k}}}\right) \text { as } k \rightarrow \infty,
\end{aligned}
$$

due to the fact that (2.28) gives

$$
\frac{\left|\mu_{2, k}-\mu_{1, k}\right| \varepsilon_{k}^{2}}{\left\|\bar{u}_{2, k}-\bar{u}_{1, k}\right\|_{L^{\infty}}} \leq \frac{\lambda^{4} a_{k}}{2\left(a^{*}\right)^{2} \varepsilon_{k}^{2}} \int_{\mathbb{R}^{2}}\left(\bar{u}_{2, k}^{2}+\bar{u}_{1, k}^{2}\right)\left(\bar{u}_{2, k}+\bar{u}_{1, k}\right)\left|\bar{\xi}_{k}\right| \leq M
$$


where the constants $M>0$ is independent of $k$. Because $h(x)$ is homogeneous of degree $p$, it then follows from (2.40) that for small $\delta>0$,

$$
\begin{aligned}
& o\left(e^{-\frac{C \delta}{\varepsilon_{k}}}\right)= \varepsilon_{k}^{2} \int_{B_{\delta}\left(x_{2, k}\right)} \frac{\partial h(x)}{\partial x_{j}}\left[\bar{u}_{2, k}(x)+\bar{u}_{1, k}(x)\right] \bar{\xi}_{k}(x) d x \\
&= \frac{\varepsilon_{k}^{3}}{\lambda} \int_{B_{\frac{\lambda \delta}{\varepsilon_{k}}}(0)} \frac{\partial}{\partial y_{j}} h\left(\frac{\varepsilon_{k}}{\lambda} y+x_{2, k}\right) \bar{\xi}_{k}\left(\frac{\varepsilon_{k}}{\lambda} y+x_{2, k}\right) \\
& \cdot\left[\bar{u}_{2, k}\left(\frac{\varepsilon_{k}}{\lambda} y+x_{2, k}\right)+\bar{u}_{1, k}\left(\frac{\varepsilon_{k}}{\lambda} y+x_{2, k}\right)\right] d y \\
&=\frac{\varepsilon_{k}^{p+3}}{\lambda^{p+1}}\left[\int_{B_{\frac{\lambda \delta}{\varepsilon_{k}}}(0)} \frac{\partial}{\partial y_{j}} h\left(y+\frac{\lambda x_{2, k}}{\varepsilon_{k}}\right) \bar{\xi}_{k}\left(\frac{\varepsilon_{k}}{\lambda} y+x_{2, k}\right)\right. \\
&\left.\cdot\left[\bar{u}_{2, k}\left(\frac{\varepsilon_{k}}{\lambda} y+x_{2, k}\right)+\bar{u}_{1, k}\left(\frac{\varepsilon_{k}}{\lambda} y+x_{1, k}\right)\right] d y+o(1)\right]
\end{aligned}
$$

as $k \rightarrow \infty$. Applying (1.14), we thus derive from (2.6), (2.32) and (2.44) that

$$
\begin{aligned}
0=2 \int_{\mathbb{R}^{2}} \frac{\partial h\left(x+y_{0}\right)}{\partial x_{j}} w \xi_{0} & =2 \int_{\mathbb{R}^{2}} \frac{\partial h\left(x+y_{0}\right)}{\partial x_{j}} w\left[b_{0}(w+x \cdot \nabla w)+\sum_{i=1}^{2} b_{i} \frac{\partial w}{\partial x_{i}}\right] \\
& =b_{0} \int_{\mathbb{R}^{2}} \frac{\partial h\left(x+y_{0}\right)}{\partial x_{j}}\left(x \cdot \nabla w^{2}\right)-\sum_{i=1}^{2} b_{i} \int_{\mathbb{R}^{2}} \frac{\partial^{2} h\left(x+y_{0}\right)}{\partial x_{j} \partial x_{i}} w^{2},
\end{aligned}
$$

where $j=1,2$, which thus implies (2.37).

We next derive $b_{0}=0$. Using the integration by parts, we note that

$$
\begin{aligned}
& -\varepsilon_{k}^{2} \int_{B_{\delta}\left(x_{2, k}\right)}\left[\left(x-x_{2, k}\right) \cdot \nabla \bar{u}_{i, k}\right] \Delta \bar{u}_{i, k} \\
= & -\varepsilon_{k}^{2} \int_{\partial B_{\delta}\left(x_{2, k}\right)} \frac{\partial \bar{u}_{i, k}}{\partial \nu}\left[\left(x-x_{2, k}\right) \cdot \nabla \bar{u}_{i, k}\right]+\varepsilon_{k}^{2} \int_{B_{\delta}\left(x_{2, k}\right)} \nabla \bar{u}_{i, k} \nabla\left[\left(x-x_{2, k}\right) \cdot \nabla \bar{u}_{i, k}\right] \\
= & -\varepsilon_{k}^{2} \int_{\partial B_{\delta}\left(x_{2, k}\right)} \frac{\partial \bar{u}_{i, k}}{\partial \nu}\left[\left(x-x_{2, k}\right) \cdot \nabla \bar{u}_{i, k}\right]+\frac{\varepsilon_{k}^{2}}{2} \int_{\partial B_{\delta}\left(x_{2, k}\right)}\left[\left(x-x_{2, k}\right) \cdot \nu\right]\left|\nabla \bar{u}_{i, k}\right|^{2} .
\end{aligned}
$$

Multiplying (2.25) by $\left(x-x_{2, k}\right) \cdot \nabla \bar{u}_{i, k}$, where $i=1,2$, and integrating over $B_{\delta}\left(x_{2, k}\right)$, 
where $\delta>0$ is small as before, we deduce that for $i=1,2$,

$$
\begin{aligned}
& -\varepsilon_{k}^{2} \int_{B_{\delta}\left(x_{2, k}\right)}\left[\left(x-x_{2, k}\right) \cdot \nabla \bar{u}_{i, k}\right] \Delta \bar{u}_{i, k} \\
= & \varepsilon_{k}^{2} \int_{B_{\delta}\left(x_{2, k}\right)}\left[\mu_{i, k}-h(x)\right] \bar{u}_{i, k}\left[\left(x-x_{2, k}\right) \cdot \nabla \bar{u}_{i, k}\right] \\
& +\frac{\lambda^{2} a_{k}}{a^{*}} \int_{B_{\delta}\left(x_{2, k}\right)} \bar{u}_{i, k}^{3}\left[\left(x-x_{2, k}\right) \cdot \nabla \bar{u}_{i, k}\right] \\
= & -\frac{\varepsilon_{k}^{2}}{2} \int_{B_{\delta}\left(x_{2, k}\right)} \bar{u}_{i, k}^{2}\left\{2\left[\mu_{i, k}-h(x)\right]-\left(x-x_{2, k}\right) \cdot \nabla h(x)\right\} \\
& +\frac{\varepsilon_{k}^{2}}{2} \int_{\partial B_{\delta}\left(x_{2, k}\right)} \bar{u}_{i, k}^{2}\left[\mu_{i, k}-h(x)\right]\left(x-x_{2, k}\right) \nu d S \\
& -\frac{\lambda^{2} a_{k}}{2 a^{*}} \int_{B_{\delta}\left(x_{2, k}\right)} \bar{u}_{i, k}^{4}+\frac{\lambda^{2} a_{k}}{4 a^{*}} \int_{\partial B_{\delta}\left(x_{2, k}\right)} \bar{u}_{i, k}^{4}\left(x-x_{2, k}\right) \nu d S \\
= & -\mu_{i, k} \varepsilon_{k}^{2} \int_{\mathbb{R}^{2}} \bar{u}_{i, k}^{2}+\frac{2+p}{2} \varepsilon_{k}^{2} \int_{\mathbb{R}^{2}} h(x) \bar{u}_{i, k}^{2}-\frac{\lambda^{2} a_{k}}{2 a^{*}} \int_{\mathbb{R}^{2}} \bar{u}_{i, k}^{4}+I_{i},
\end{aligned}
$$

where the lower order term $I_{i}$ satisfies

$$
\begin{aligned}
I_{i}= & \mu_{i, k} \varepsilon_{k}^{2} \int_{\mathbb{R}^{2} \backslash B_{\delta}\left(x_{2, k}\right)} \bar{u}_{i, k}^{2}-\frac{2+p}{2} \varepsilon_{k}^{2} \int_{\mathbb{R}^{2} \backslash B_{\delta}\left(x_{2, k}\right)} h(x) \bar{u}_{i, k}^{2} \\
& +\frac{\lambda^{2} a_{k}}{2 a^{*}} \int_{\mathbb{R}^{2} \backslash B_{\delta}\left(x_{2, k}\right)} \bar{u}_{i, k}^{4}-\frac{1}{2} \varepsilon_{k}^{2} \int_{B_{\delta}\left(x_{2, k}\right)} \bar{u}_{i, k}^{2}\left[x_{2, k} \cdot \nabla h(x)\right] \\
& +\frac{\varepsilon_{k}^{2}}{2} \int_{\partial B_{\delta}\left(x_{2, k}\right)} \bar{u}_{i, k}^{2}\left[\mu_{i, k}-h(x)\right]\left(x-x_{2, k}\right) \nu d S \\
& +\frac{\lambda^{2} a_{k}}{4 a^{*}} \int_{\partial B_{\delta}\left(x_{2, k}\right)} \bar{u}_{i, k}^{4}\left(x-x_{2, k}\right) \nu d S, \quad i=1,2 .
\end{aligned}
$$

Since it follows from (2.17) that

$$
-\mu_{i, k} \varepsilon_{k}^{2} \int_{\mathbb{R}^{2}} \bar{u}_{i, k}^{2}-\frac{\lambda^{2} a_{k}}{2 a^{*}} \int_{\mathbb{R}^{2}} \bar{u}_{i, k}^{4}=-\frac{a^{*} \varepsilon_{k}^{4}}{\lambda^{2}}\left[\mu_{i, k}+\frac{a_{k}}{2} \int_{\mathbb{R}^{2}} u_{i, k}^{4}\right]=-\frac{a^{*} \varepsilon_{k}^{4}}{\lambda^{2}} e\left(a_{k}\right),
$$

we reduce from (2.45)-(2.47) that

$$
\begin{aligned}
\frac{a^{*} \varepsilon_{k}^{4}}{\lambda^{2}} e\left(a_{k}\right)-\frac{2+p}{2} \varepsilon_{k}^{2} \int_{\mathbb{R}^{2}} h(x) \bar{u}_{i, k}^{2}= & I_{i}+\varepsilon_{k}^{2} \int_{\partial B_{\delta}\left(x_{2, k}\right)} \frac{\partial \bar{u}_{i, k}}{\partial \nu}\left[\left(x-x_{2, k}\right) \cdot \nabla \bar{u}_{i, k}\right] \\
& -\frac{\varepsilon_{k}^{2}}{2} \int_{\partial B_{\delta}\left(x_{2, k}\right)}\left[\left(x-x_{2, k}\right) \cdot \nu\right]\left|\nabla \bar{u}_{i, k}\right|^{2}, \quad i=1,2,
\end{aligned}
$$

which implies that

$$
-\frac{2+p}{2} \varepsilon_{k}^{2} \int_{\mathbb{R}^{2}} h(x)\left[\bar{u}_{2, k}+\bar{u}_{1, k}\right] \bar{\xi}_{k}=T_{k}
$$


Here the term $T_{k}$ satisfies that for small $\delta>0$,

$$
\begin{aligned}
T_{k}= & \frac{I_{2}-I_{1}}{\left\|\bar{u}_{2, k}-\bar{u}_{1, k}\right\|_{L^{\infty}}}-\frac{\varepsilon_{k}^{2}}{2} \int_{\partial B_{\delta}\left(x_{2, k}\right)}\left[\left(x-x_{2, k}\right) \cdot \nu\right]\left(\nabla \bar{u}_{2, k}+\nabla \bar{u}_{1, k}\right) \nabla \bar{\xi}_{k} \\
& +\varepsilon_{k}^{2} \int_{\partial B_{\delta}\left(x_{2, k}\right)}\left\{\left[\left(x-x_{2, k}\right) \cdot \nabla \bar{u}_{2, k}\right]\left(\nu \cdot \nabla \bar{\xi}_{k}\right)+\left(\nu \cdot \nabla \bar{u}_{1, k}\right)\left[\left(x-x_{2, k}\right) \cdot \nabla \bar{\xi}_{k}\right]\right\} \\
= & \frac{I_{2}-I_{1}}{\left\|\bar{u}_{2, k}-\bar{u}_{1, k}\right\|_{L^{\infty}}}+o\left(e^{-\frac{C \delta}{\varepsilon_{k}}}\right) \text { as } k \rightarrow \infty,
\end{aligned}
$$

due to (2.22) and (2.29), where the second equality follows by applying the argument of estimating (2.41).

Using the arguments of estimating (2.41) and (2.42), along with the exponential decay of $\bar{u}_{i, k}$, we also derive that for small $\delta>0$,

$$
\begin{aligned}
& \frac{I_{2}-I_{1}}{\left\|\bar{u}_{2, k}-\bar{u}_{1, k}\right\|_{L^{\infty}}} \\
& =\mu_{2, k} \varepsilon_{k}^{2} \int_{\mathbb{R}^{2} \backslash B_{\delta}\left(x_{2, k}\right)}\left(\bar{u}_{2, k}+\bar{u}_{1, k}\right) \bar{\xi}_{k}-\frac{2+p}{2} \varepsilon_{k}^{2} \int_{\mathbb{R}^{2} \backslash B_{\delta}\left(x_{2, k}\right)} h(x)\left(\bar{u}_{2, k}+\bar{u}_{1, k}\right) \bar{\xi}_{k} \\
& +\frac{\lambda^{2} a_{k}}{2 a^{*}} \int_{\mathbb{R}^{2} \backslash B_{\delta}\left(x_{2, k}\right)}\left(\bar{u}_{2, k}^{2}+\bar{u}_{1, k}^{2}\right)\left(\bar{u}_{2, k}+\bar{u}_{1, k}\right) \bar{\xi}_{k} \\
& +\frac{\left(\mu_{2, k}-\mu_{1, k}\right) \varepsilon_{k}^{2}}{\left\|\bar{u}_{2, k}-\bar{u}_{1, k}\right\|_{L^{\infty}}} \int_{\mathbb{R}^{2} \backslash B_{\delta}\left(x_{2, k}\right)} \bar{u}_{1, k}^{2}-\frac{1}{2} \varepsilon_{k}^{2} \int_{B_{\delta}\left(x_{2, k}\right)}\left[x_{2, k} \cdot \nabla h(x)\right]\left(\bar{u}_{2, k}+\bar{u}_{1, k}\right) \bar{\xi}_{k} \\
& +\frac{\lambda^{2} a_{k}}{4 a^{*}} \int_{\partial B_{\delta}\left(x_{2, k}\right)}\left(\bar{u}_{2, k}^{2}+\bar{u}_{1, k}^{2}\right)\left(\bar{u}_{2, k}+\bar{u}_{1, k}\right) \bar{\xi}_{k}\left(x-x_{2, k}\right) \nu d S \\
& -\frac{\varepsilon_{k}^{2}}{2} \int_{\partial B_{\delta}\left(x_{2, k}\right)}\left(\bar{u}_{2, k}+\bar{u}_{1, k}\right) \bar{\xi}_{k} h(x)\left(x-x_{2, k}\right) \nu d S \\
& +\frac{\mu_{2, k} \varepsilon_{k}^{2}}{2} \int_{\partial B_{\delta}\left(x_{2, k}\right)}\left(\bar{u}_{2, k}+\bar{u}_{1, k}\right) \bar{\xi}_{k}\left(x-x_{2, k}\right) \nu d S \\
& +\frac{\left(\mu_{2, k}-\mu_{1, k}\right) \varepsilon_{k}^{2}}{2\left\|\bar{u}_{2, k}-\bar{u}_{1, k}\right\|_{L^{\infty}}} \int_{\partial B_{\delta}\left(x_{2, k}\right)} \bar{u}_{1, k}^{2}\left(x-x_{2, k}\right) \nu d S \\
& =\frac{\left(\mu_{2, k}-\mu_{1, k}\right) \varepsilon_{k}^{2}}{\left\|\bar{u}_{2, k}-\bar{u}_{1, k}\right\|_{L^{\infty}}}\left[\int_{\mathbb{R}^{2} \backslash B_{\delta}\left(x_{2, k}\right)} \bar{u}_{1, k}^{2}+\frac{1}{2} \int_{\partial B_{\delta}\left(x_{2, k}\right)} \bar{u}_{1, k}^{2}\left(x-x_{2, k}\right) \nu d S\right] \\
& -\frac{1}{2} \varepsilon_{k}^{2} \int_{B_{\delta}\left(x_{2, k}\right)}\left[x_{2, k} \cdot \nabla h(x)\right]\left(\bar{u}_{2, k}+\bar{u}_{1, k}\right) \bar{\xi}_{k}+o\left(e^{-\frac{C \delta}{\varepsilon_{k}}}\right) \text { as } k \rightarrow \infty \text {. }
\end{aligned}
$$

Note from (2.43) that

$$
\frac{\left(\mu_{2, k}-\mu_{1, k}\right) \varepsilon_{k}^{2}}{\left\|\bar{u}_{2, k}-\bar{u}_{1, k}\right\|_{L^{\infty}}}\left[\int_{\mathbb{R}^{2} \backslash B_{\delta}\left(x_{2, k}\right)} \bar{u}_{1, k}^{2}+\frac{1}{2} \int_{\partial B_{\delta}\left(x_{2, k}\right)} \bar{u}_{1, k}^{2}\left(x-x_{2, k}\right) \nu d S\right]=O\left(e^{-\frac{C \delta}{\varepsilon_{k}}}\right)
$$

as $k \rightarrow \infty$, where the constant $C>0$ is independent of $k$. Moreover, we follow from the 
first identity of (2.44) that

$$
\begin{aligned}
& \frac{1}{2} \varepsilon_{k}^{2} \int_{B_{\delta}\left(x_{2, k}\right)}\left[x_{2, k} \cdot \nabla h(x)\right]\left(\bar{u}_{2, k}+\bar{u}_{1, k}\right) \bar{\xi}_{k} \\
= & \frac{1}{2} \varepsilon_{k}^{2} \sum_{i=1}^{2} x_{2, k}^{i} \int_{B_{\delta}\left(x_{2, k}\right)} \frac{\partial h(x)}{\partial x_{i}}\left[\bar{u}_{2, k}(x)+\bar{u}_{1, k}(x)\right] \bar{\xi}_{k}(x) d x \\
= & o\left(e^{-\frac{C \delta}{\varepsilon_{k}}}\right) \text { as } k \rightarrow \infty,
\end{aligned}
$$

where we denote $x_{2, k}=\left(x_{2, k}^{1}, x_{2, k}^{2}\right)$. Therefore, we deduce from (2.49)-(2.52) that

$$
T_{k}=o\left(\varepsilon_{k}^{4+p}\right) \text { as } k \rightarrow \infty .
$$

Further, we obtain from (2.48) that

$$
\begin{aligned}
o\left(\varepsilon_{k}^{4+p}\right)= & -\frac{2+p}{2} \varepsilon_{k}^{2} \int_{\mathbb{R}^{2}} h(x)\left[\bar{u}_{2, k}+\bar{u}_{1, k}\right] \bar{\xi}_{k} \\
= & -\frac{2+p}{2 \lambda^{2}} \varepsilon_{k}^{4} \int_{\mathbb{R}^{2}} h\left(\frac{\varepsilon_{k}}{\lambda} x+x_{2, k}\right)\left[\bar{u}_{2, k}\left(\frac{\varepsilon_{k}}{\lambda} x+x_{2, k}\right)+\bar{u}_{1, k}\left(\frac{\varepsilon_{k}}{\lambda} x+x_{1, k}\right)\right] \xi_{k}(x) d x \\
& -\frac{2+p}{2 \lambda^{2}} \varepsilon_{k}^{4} \int_{\mathbb{R}^{2}} h\left(\frac{\varepsilon_{k}}{\lambda} x+x_{2, k}\right)\left[\bar{u}_{1, k}\left(\frac{\varepsilon_{k}}{\lambda} x+x_{2, k}\right)-\bar{u}_{1, k}\left(\frac{\varepsilon_{k}}{\lambda} x+x_{1, k}\right)\right] \xi_{k}(x) d x \\
= & -\frac{2+p}{2 \lambda^{2+p}} \varepsilon_{k}^{4+p} \int_{\mathbb{R}^{2}} h\left(x+\frac{\lambda x_{2, k}}{\varepsilon_{k}}\right)\left[\bar{u}_{2, k}\left(\frac{\varepsilon_{k}}{\lambda} x+x_{2, k}\right)+\bar{u}_{1, k}\left(\frac{\varepsilon_{k}}{\lambda} x+x_{1, k}\right)\right] \xi_{k}(x) d x \\
& +O\left(\varepsilon_{k}^{4+p}\left|x_{2, k}-x_{1, k}\right|\right) \text { as } k \rightarrow \infty .
\end{aligned}
$$

Since $\left(x+y_{0}\right) \cdot \nabla h\left(x+y_{0}\right)=p h\left(x+y_{0}\right)$, by Proposition 2.1 and Step 1, we thus obtain from (1.14) and above that

$$
\begin{aligned}
0=2 \int_{\mathbb{R}^{2}} h\left(x+y_{0}\right) w \xi_{0} & =2 b_{0} \int_{\mathbb{R}^{2}} h\left(x+y_{0}\right) w(w+x \cdot \nabla w)+\sum_{i=1}^{2} b_{i} \int_{\mathbb{R}^{2}} h\left(x+y_{0}\right) \frac{\partial w^{2}}{\partial x_{i}} \\
& =2 b_{0}\left[\int_{\mathbb{R}^{2}} h\left(x+y_{0}\right) w^{2}+\frac{1}{2} \int_{\mathbb{R}^{2}} h\left(x+y_{0}\right)\left(x \cdot \nabla w^{2}\right)\right] \\
& =2 b_{0}\left\{\int_{\mathbb{R}^{2}} h\left(x+y_{0}\right) w^{2}-\frac{1}{2} \int_{\mathbb{R}^{2}} w^{2}\left[2 h\left(x+y_{0}\right)+x \cdot \nabla h\left(x+y_{0}\right)\right]\right\} \\
& =-p b_{0} \int_{\mathbb{R}^{2}} h\left(x+y_{0}\right) w^{2}+b_{0} \int_{\mathbb{R}^{2}} w^{2}\left[y_{0} \cdot \nabla h\left(x+y_{0}\right)\right] \\
& =-p b_{0} \int_{\mathbb{R}^{2}} h\left(x+y_{0}\right) w^{2},
\end{aligned}
$$

which therefore implies that $b_{0}=0$.

By the non-degeneracy assumption (1.14), setting $b_{0}=0$ into (2.37) then yields that $b_{1}=b_{2}=0$, and Step 2 is therefore proved.

Step 3. $\xi_{0} \equiv 0$ cannot occur.

Finally, let $y_{k}$ be a point satisfying $\left|\xi_{k}\left(y_{k}\right)\right|=\left\|\xi_{k}\right\|_{L^{\infty}\left(\mathbb{R}^{2}\right)}=1$. By the same argument as employed in proving Lemma 3.1 in next section, applying the maximum principle to (2.33) yields that $\left|y_{k}\right| \leq C$ uniformly in $k$. Therefore, we conclude that $\xi_{k} \rightarrow \xi_{0} \not \equiv 0$ uniformly on $\mathbb{R}^{2}$, which however contradicts to the fact that $\xi_{0} \equiv 0$ on $\mathbb{R}^{2}$. This completes the proof of Theorem 1.1. 


\section{$3 \quad$ Refined Spike Profiles}

In the following two sections, we shall derive the refined spike profiles of positive minimizers $u_{k}=u_{a_{k}}$ for $e\left(a_{k}\right)$ as $a_{k} \nearrow a^{*}$. The purpose of this section is to prove Theorem [1.2. Recall first that $u_{k}$ satisfies the Euler-Lagrange equation (2.16). Under the assumptions of Proposition 2.1, for convenience, we denote

$$
\varepsilon_{k}=\left(a^{*}-a_{k}\right)^{\frac{1}{2+p}}>0, \quad \alpha_{k}:=\varepsilon_{k}^{2+p}>0 \text { and } \beta_{k}:=1+\frac{\mu_{k} \varepsilon_{k}^{2}}{\lambda^{2}},
$$

where $\mu_{k} \in \mathbb{R}$ is the Lagrange multiplier of the equation (2.16), so that

$$
\alpha_{k} \rightarrow 0 \text { and } \beta_{k} \rightarrow 0 \text { as } k \rightarrow \infty
$$

where (2.19) is used. In order to discuss the refined spike profiles of $u_{k}$ as $k \rightarrow \infty$, the key is thus to obtain the refined estimate of $\mu_{k}$ (equivalently $\beta_{k}$ ) in terms of $\varepsilon_{k}$.

We next define

$$
w_{k}(x):=\bar{u}_{k}(x)-w(x):=\frac{\sqrt{a^{*}} \varepsilon_{k}}{\lambda} u_{k}\left(\frac{\varepsilon_{k}}{\lambda} x+x_{k}\right)-w(x),
$$

where $x_{k}$ is the unique maximum point of $u_{k}$, so that $w_{k}(x) \rightarrow 0$ uniformly in $\mathbb{R}^{2}$ by Proposition 2.1. By applying (2.16), direct calculations then give that $\bar{u}_{k}$ satisfies

$$
-\Delta \bar{u}_{k}(x)+\frac{\varepsilon_{k}^{2}}{\lambda^{2}} V\left(\frac{\varepsilon_{k}}{\lambda} x+x_{k}\right) \bar{u}_{k}(x)=\frac{\mu_{k} \varepsilon_{k}^{2}}{\lambda^{2}} \bar{u}_{k}(x)+\frac{a_{k}}{a^{*}} \bar{u}_{k}^{3}(x) \text { in } \mathbb{R}^{2}
$$

Relating to the operator $\mathcal{L}:=-\Delta+\left(1-3 w^{2}\right)$ in $\mathbb{R}^{2}$, we also denote the linearized operator

$$
\mathcal{L}_{k}:=-\Delta+\left[1-\left(\bar{u}_{k}^{2}+\bar{u}_{k} w+w^{2}\right)\right] \text { in } \mathbb{R}^{2},
$$

so that $w_{k}$ satisfies

$$
\begin{aligned}
\mathcal{L}_{k} w_{k}(x)= & -\alpha_{k}\left[\frac{1}{a^{*}} \bar{u}_{k}^{3}(x)+\frac{1}{\lambda^{2+p}} g\left(\frac{\varepsilon_{k} x}{\lambda}+x_{k}\right) h\left(x+\frac{\lambda x_{k}}{\varepsilon_{k}}\right) \bar{u}_{k}(x)\right] \\
& +\beta_{k} \bar{u}_{k}(x) \text { in } \mathbb{R}^{2}, \quad \nabla w_{k}(0)=0,
\end{aligned}
$$

where $V(x)=g(x) h(x)$ satisfies the assumptions of Proposition 2.1 and the coefficients $\alpha_{k}>0$ and $\beta_{k}>0$ are as in (3.1). Define

$$
\begin{array}{r}
\mathcal{L}_{k} \psi_{1, k}(x)=-\alpha_{k}\left[\frac{1}{\lambda^{2+p}} g\left(\frac{\varepsilon_{k} x}{\lambda}+x_{k}\right) h\left(x+\frac{\lambda x_{k}}{\varepsilon_{k}}\right) \bar{u}_{k}(x)\right. \\
\left.+\frac{1}{a^{*}} \bar{u}_{k}^{3}(x)\right] \text { in } \mathbb{R}^{2}, \quad \nabla \psi_{1, k}(0)=0, \\
\mathcal{L}_{k} \psi_{2, k}(x)=\beta_{k} \bar{u}_{k}(x) \text { in } \mathbb{R}^{2}, \quad \nabla \psi_{2, k}(0)=0,
\end{array}
$$

so that the solution $w_{k}(x)$ of (3.3) satisfies

$$
w_{k}(x):=\psi_{1, k}(x)+\psi_{2, k}(x) \text { in } \mathbb{R}^{2} .
$$

We first employ Proposition 2.1 to address the following estimates of $w_{k}$ as $k \rightarrow \infty$.

Lemma 3.1. Under the assumptions of Proposition [2.1, where $V(x)=g(x) h(x)$, we have 
1. $\psi_{1, k}(x)$ satisfies

$$
\psi_{1, k}(x)=\alpha_{k} \psi_{1}(x)+o\left(\alpha_{k}\right) \text { as } k \rightarrow \infty,
$$

where $\psi_{1}(x) \in C^{2}\left(\mathbb{R}^{2}\right) \cap L^{\infty}\left(\mathbb{R}^{2}\right)$ solves uniquely

$$
\nabla \psi_{1}(0)=0, \quad \mathcal{L} \psi_{1}(x)=-\frac{1}{a^{*}} w^{3}(x)-\frac{g(0)}{\lambda^{2+p}} h\left(x+y_{0}\right) w(x) \text { in } \mathbb{R}^{2},
$$

where $y_{0} \in \mathbb{R}^{2}$ is given by (1.9).

2. $\psi_{2, k}(x)$ satisfies

$$
\psi_{2, k}(x)=\beta_{k} \psi_{2}(x)+o\left(\beta_{k}\right) \text { as } k \rightarrow \infty,
$$

where $\psi_{2}(x)$ solves uniquely

$$
\nabla \psi_{2}(0)=0, \quad \mathcal{L} \psi_{2}(x)=w(x) \text { in } \mathbb{R}^{2},
$$

i.e., $\psi_{2}(x) \in C^{2}\left(\mathbb{R}^{2}\right) \cap L^{\infty}\left(\mathbb{R}^{2}\right)$ satisfies

$$
\psi_{2}=-\frac{1}{2}(w+x \cdot \nabla w) .
$$

3. $w_{k}$ satisfies

$$
w_{k}(x):=\alpha_{k} \psi_{1}(x)+\beta_{k} \psi_{2}(x)+o\left(\alpha_{k}+\beta_{k}\right) \text { as } k \rightarrow \infty .
$$

Proof. 1. We first derive $\left|\psi_{1, k}\right| \leq C \alpha_{k}$ in $\mathbb{R}^{2}$ by contradiction. On the contrary, we assume that

$$
\lim _{k \rightarrow \infty} \frac{\left\|\psi_{1, k}\right\|_{L^{\infty}}}{\alpha_{k}}=\infty
$$

Set $\bar{\psi}_{1, k}=\frac{\psi_{1, k}}{\left\|\psi_{1, k}\right\|_{L^{\infty}}}$ so that $\left\|\bar{\psi}_{1, k}\right\|_{L^{\infty}}=1$. Following (3.4), $\bar{\psi}_{1, k}$ then satisfies

$$
\begin{aligned}
& -\Delta \bar{\psi}_{1, k}+\left[1-\left(\bar{u}_{k}^{2}+\bar{u}_{k} w+w^{2}\right)\right] \bar{\psi}_{1, k} \\
= & -\frac{\alpha_{k}}{\left\|\psi_{1, k}\right\|_{\infty}}\left[\frac{1}{\lambda^{2+p}} g\left(\frac{\varepsilon_{k} x}{\lambda}+x_{k}\right) h\left(x+\frac{\lambda x_{k}}{\varepsilon_{k}}\right) \bar{u}_{k}(x)+\frac{1}{a^{*}} \bar{u}_{k}^{3}(x)\right] \text { in } \mathbb{R}^{2} .
\end{aligned}
$$

Let $y_{k}$ be the global maximum point of $\bar{\psi}_{1, k}$ so that $\bar{\psi}_{1, k}\left(y_{k}\right)=\max _{y \in \mathbb{R}^{2}} \frac{\psi_{1, k}(y)}{\left\|\psi_{1, k}\right\|_{L^{\infty}}}=1$. Since both $\bar{u}_{k}$ and $w$ decay exponentially in view of (2.7), using the maximum principle we derive from (3.13) that $\left|y_{k}\right| \leq C$ uniformly in $k$.

On the other hand, applying the usual elliptic regularity theory, there exists a subsequence, still denoted by $\left\{\bar{\psi}_{1, k}\right\}$, of $\left\{\bar{\psi}_{1, k}\right\}$ such that $\bar{\psi}_{1, k} \rightarrow \bar{\psi}_{1}$ weakly in $H^{1}\left(\mathbb{R}^{2}\right)$ and strongly in $L_{l o c}^{q}\left(\mathbb{R}^{2}\right)$ for all $q \in[2, \infty)$. Here $\bar{\psi}_{1}$ satisfies

$$
\nabla \bar{\psi}_{1}(0)=0, \quad \mathcal{L} \bar{\psi}_{1}(x)=0 \text { in } \mathbb{R}^{2},
$$

which implies that $\bar{\psi}_{1}=\sum_{i=1}^{2} c_{i} \frac{\partial w}{\partial y_{i}}$. Since $\nabla \bar{\psi}_{1}(0)=0$, we obtain that $c_{1}=c_{2}=0$. Thus, we have $\bar{\psi}_{1}(y) \equiv 0$ in $\mathbb{R}^{2}$, which however contradicts to the fact that $1=\bar{\psi}_{1, k}\left(y_{k}\right) \rightarrow$ $\bar{\psi}_{1}\left(\bar{y}_{0}\right)$ for some $\bar{y}_{0} \in \mathbb{R}^{2}$ by passing to a subsequence if necessary. Therefore, we have $\left|\psi_{1, k}\right| \leq C \alpha_{k}$ in $\mathbb{R}^{2}$.

We next set $\phi_{1, k}(x)=\psi_{1, k}(x)-\alpha_{k} \psi_{1}(x)$, where $\psi_{1}(x) \in C^{2}\left(\mathbb{R}^{2}\right) \cap L^{\infty}\left(\mathbb{R}^{2}\right)$ is a solution of (3.7). Then either $\phi_{1, k}(x)=O\left(\alpha_{k}\right)$ or $\phi_{1, k}(x)=o\left(\alpha_{k}\right)$ as $k \rightarrow \infty$, and $\phi_{1, k}$ satisfies

$$
\nabla \phi_{1, k}(0)=0, \quad-\Delta \phi_{1, k}+\left[1-\left(\bar{u}_{k}^{2}+\bar{u}_{k} w+w^{2}\right)\right] \phi_{1, k}=-\alpha_{k} f_{k}(x) \text { in } \mathbb{R}^{2}
$$


where $f_{k}(x)$ satisfies

$$
\begin{aligned}
f_{k}(x)= & \left(2 w^{2}-\bar{u}_{k}^{2}-\bar{u}_{k} w\right) \psi_{1}(x)+\frac{1}{a^{*}}\left(\bar{u}_{k}^{3}(x)-w^{3}(x)\right) \\
& +\frac{1}{\lambda^{2+p}}\left[g\left(\frac{\varepsilon_{k} x}{\lambda}+x_{k}\right) h\left(x+\frac{\lambda x_{k}}{\varepsilon_{k}}\right) \bar{u}_{k}(x)-g(0) h\left(x+y_{0}\right) w(x)\right] .
\end{aligned}
$$

One can note that $f_{k}(x) \rightarrow 0$ uniformly as $k \rightarrow \infty$. Therefore, applying the previous argument yields necessarily that $\phi_{1, k}(x)=o\left(\alpha_{k}\right)$ as $k \rightarrow \infty$, and the proof of (3.6) is then complete. Also, the property (2.15) gives the uniqueness of solutions for (3.7).

2. Since the proof of (3.8) is very similar to that of (3.6), we omit the details. Further, the property (2.15) gives the uniqueness of $\psi_{2}$. Also, one can check directly that $-(w+x \cdot \nabla w) / 2$ is a solution of (3.9), which therefore implies that (3.10) holds.

3. The expansion (3.11) now follows immediately from (3.5), (3.6) and (3.8), and the proof is therefore complete.

\subsection{Proof of Theorem 1.2}

The main aim of this subsection is to prove Theorem 1.2 on the refined spike behavior of positive minimizers. In this whole subsection, we assume that the potential $V(x)=$ $h(x) \in C^{2}\left(\mathbb{R}^{2}\right)$ satisfies $\lim _{|x| \rightarrow \infty} h(x)=\infty$ and (1.14), where $h(x)$ is homogeneous of degree $p \geq 2$. Following (3.1), from now on we denote for simplicity that

$$
o\left(\left[\alpha_{k}+\beta_{k}\right]^{2}\right)=o\left(\alpha_{k}^{2}\right)+o\left(\alpha_{k} \beta_{k}\right)+o\left(\beta_{k}^{2}\right) \text { as } k \rightarrow \infty,
$$

where $\alpha_{k}$ and $\beta_{k}$ are defined in (3.1). We first use Lemma 3.1 to establish the following lemmas.

Lemma 3.2. Suppose that $V(x)=h(x) \in C^{2}\left(\mathbb{R}^{2}\right)$ satisfies $\lim _{|x| \rightarrow \infty} h(x)=\infty$ and (1.14) for some $y_{0} \in \mathbb{R}^{2}$, where $h(x)$ is homogeneous of degree $p \geq 2$. Then there exists an $x_{0} \in \mathbb{R}^{2}$ such that the unique maximum point $x_{k}$ of $u_{k}$ satisfies

$$
\left|\alpha_{k}\left(\frac{\lambda x_{k}}{\varepsilon_{k}}-y_{0}\right)-\alpha_{k} \beta_{k} \frac{y_{0}}{2}\right|=\alpha_{k}^{2} O\left(\left|x_{0}\right|\right)+o\left(\left[\alpha_{k}+\beta_{k}\right]^{2}\right) \text { as } k \rightarrow \infty .
$$

Proof. Multiplying (3.7) and (3.9) by $\frac{\partial w}{\partial x_{1}}$ and then integrating over $\mathbb{R}^{2}$, respectively, we obtain from (1.14) and (2.15) that

$$
\int_{\mathbb{R}^{2}} \frac{\partial w}{\partial x_{1}} \mathcal{L} w_{k}=\int_{\mathbb{R}^{2}} \frac{\partial w}{\partial x_{1}} w=\int_{\mathbb{R}^{2}} \frac{\partial w}{\partial x_{1}} h\left(x+y_{0}\right) w=0,
$$

where $y_{0}$ is given by the assumption (1.14). Similarly, we derive from (3.3) and (3.11) that

$$
\begin{aligned}
\int_{\mathbb{R}^{2}} \frac{\partial w}{\partial x_{1}} \mathcal{L}_{k} w_{k} & =\beta_{k} \int_{\mathbb{R}^{2}} \frac{\partial w}{\partial x_{1}} \bar{u}_{k}-\alpha_{k} \int_{\mathbb{R}^{2}} \frac{\partial w}{\partial x_{1}}\left[\frac{1}{a^{*}} \bar{u}_{k}^{3}+\frac{\bar{u}_{k}}{\lambda^{2+p}} h\left(x+\frac{\lambda x_{k}}{\varepsilon_{k}}\right)\right] \\
& =\alpha_{k} \beta_{k} \int_{\mathbb{R}^{2}} \frac{\partial w}{\partial x_{1}} \psi_{1}+o\left(\alpha_{k} \beta_{k}+\beta_{k}^{2}\right)-I_{1}
\end{aligned}
$$

where the identity $\int_{\mathbb{R}^{2}} \frac{\partial w}{\partial x_{1}} \psi_{2}=0$ is used, since $\frac{\partial w}{\partial x_{1}} \psi_{2}$ is odd in $x_{1}$ by the radial symmetry 
of $\psi_{2}$. We obtain from (1.14) and (3.16) that

$$
\begin{aligned}
I_{1}= & \alpha_{k} \int_{\mathbb{R}^{2}} \frac{\partial w}{\partial x_{1}}\left[\frac{1}{a^{*}} \bar{u}_{k}^{3}+\frac{\bar{u}_{k}}{\lambda^{2+p}} h\left(x+\frac{\lambda x_{k}}{\varepsilon_{k}}\right)\right] \\
= & \alpha_{k} \int_{\mathbb{R}^{2}} \frac{\partial w}{\partial x_{1}}\left\{\frac{1}{a^{*}}\left(\bar{u}_{k}^{3}-w^{3}\right)+\frac{1}{\lambda^{2+p}}\left[h\left(x+\frac{\lambda x_{k}}{\varepsilon_{k}}\right) \bar{u}_{k}-h\left(x+y_{0}\right) w\right]\right\} \\
= & \frac{\alpha_{k}}{a^{*}} \int_{\mathbb{R}^{2}} \frac{\partial w}{\partial x_{1}} w_{k}\left(3 w^{2}+3 w w_{k}+w_{k}^{2}\right) \\
& +\frac{\alpha_{k}}{\lambda^{2+p}} \int_{\mathbb{R}^{2}} \frac{\partial w}{\partial x_{1}}\left[h\left(x+\frac{\lambda x_{k}}{\varepsilon_{k}}\right) \bar{u}_{k}-h\left(x+y_{0}\right) w\right] \\
= & \frac{3 \alpha_{k}^{2}}{a^{*}} \int_{\mathbb{R}^{2}} \frac{\partial w}{\partial x_{1}} w^{2} \psi_{1}+o\left(\alpha_{k}^{2}+\alpha_{k} \beta_{k}\right)+I_{2},
\end{aligned}
$$

where we have used the identity $\int_{\mathbb{R}^{2}} \frac{\partial w}{\partial x_{1}} w^{2} \psi_{2}=0$, since $\frac{\partial w}{\partial x_{1}} w^{2} \psi_{2}$ is odd in $x_{1}$ by the radial symmetry of $\psi_{2}$. Further, applying (3.11) and (3.16) yields that

$$
\begin{aligned}
\frac{\lambda^{2+p}}{\alpha_{k}} I_{2}= & \int_{\mathbb{R}^{2}} \frac{\partial w}{\partial x_{1}}\left\{h\left(x+\frac{\lambda x_{k}}{\varepsilon_{k}}\right)\left[\bar{u}_{k}-w\right]+\left[h\left(x+\frac{\lambda x_{k}}{\varepsilon_{k}}\right)-h\left(x+y_{0}\right)\right] w\right\} \\
= & \int_{\mathbb{R}^{2}} \frac{\partial w}{\partial x_{1}} h\left(x+y_{0}\right) w_{k}+o\left(\alpha_{k}+\beta_{k}\right) \\
& +\int_{\mathbb{R}^{2}} \frac{\partial w}{\partial x_{1}}\left[\left(\frac{\lambda x_{k}}{\varepsilon_{k}}-y_{0}\right) \cdot \nabla h\left(x+y_{0}\right)\right] w+o\left(\left|\frac{\lambda x_{k}}{\varepsilon_{k}}-y_{0}\right|\right) \\
= & \alpha_{k} \int_{\mathbb{R}^{2}} \frac{\partial w}{\partial x_{1}} h\left(x+y_{0}\right) \psi_{1}+\beta_{k} \int_{\mathbb{R}^{2}} \frac{\partial w}{\partial x_{1}} h\left(x+y_{0}\right) \psi_{2} \\
& +\int_{\mathbb{R}^{2}} \frac{\partial w}{\partial x_{1}}\left[\left(\frac{\lambda x_{k}}{\varepsilon_{k}}-y_{0}\right) \cdot \nabla h\left(x+y_{0}\right)\right] w+o\left(\alpha_{k}+\left|\frac{\lambda x_{k}}{\varepsilon_{k}}-y_{0}\right|+\beta_{k}\right),
\end{aligned}
$$

where (2.6) is used for the second identity. We thus get that

$$
\begin{aligned}
I_{1}= & \alpha_{k}^{2}\left[\frac{3}{a^{*}} \int_{\mathbb{R}^{2}} \frac{\partial w}{\partial x_{1}} w^{2} \psi_{1}+\frac{1}{\lambda^{2+p}} \int_{\mathbb{R}^{2}} \frac{\partial w}{\partial x_{1}} h\left(x+y_{0}\right) \psi_{1}\right] \\
& +\frac{\alpha_{k} \beta_{k}}{\lambda^{2+p}} \int_{\mathbb{R}^{2}} \frac{\partial w}{\partial x_{1}} h\left(x+y_{0}\right) \psi_{2}+\frac{\alpha_{k}}{\lambda^{2+p}} \int_{\mathbb{R}^{2}} \frac{\partial w}{\partial x_{1}}\left[\left(\frac{\lambda x_{k}}{\varepsilon_{k}}-y_{0}\right) \cdot \nabla h\left(x+y_{0}\right)\right] w \\
& +o\left(\alpha_{k}\left|\frac{\lambda x_{k}}{\varepsilon_{k}}-y_{0}\right|+\left[\alpha_{k}+\beta_{k}\right]^{2}\right) .
\end{aligned}
$$

On the other hand, we obtain from (3.16) that

$$
\begin{aligned}
\int_{\mathbb{R}^{2}} \frac{\partial w}{\partial x_{1}} \mathcal{L}_{k} w_{k} & =\int_{\mathbb{R}^{2}} \frac{\partial w}{\partial x_{1}} \mathcal{L} w_{k}+\int_{\mathbb{R}^{2}} \frac{\partial w}{\partial x_{1}}\left(\mathcal{L}_{k}-\mathcal{L}\right) w_{k} \\
& =-\int_{\mathbb{R}^{2}} \frac{\partial w}{\partial x_{1}} w_{k}^{2}\left(3 w+w_{k}\right) \\
& =-3 \alpha_{k}^{2} \int_{\mathbb{R}^{2}} \frac{\partial w}{\partial x_{1}} w \psi_{1}^{2}-6 \alpha_{k} \beta_{k} \int_{\mathbb{R}^{2}} \frac{\partial w}{\partial x_{1}} w \psi_{1} \psi_{2}+o\left(\alpha_{k}^{2}+\alpha_{k} \beta_{k}\right) .
\end{aligned}
$$


Combining (3.17), (3.21) and (3.20), we now conclude from (1.14) and (3.11) that

$$
\begin{aligned}
& \frac{\alpha_{k}}{\lambda^{2+p}} \int_{\mathbb{R}^{2}} \frac{\partial w}{\partial x_{1}}\left[\left(\frac{\lambda x_{k}}{\varepsilon_{k}}-y_{0}\right) \cdot \nabla h\left(x+y_{0}\right)\right] w \\
= & \alpha_{k} \beta_{k}\left[\int_{\mathbb{R}^{2}} \frac{\partial w}{\partial x_{1}} \psi_{1}+6 \int_{\mathbb{R}^{2}} \frac{\partial w}{\partial x_{1}} w \psi_{1} \psi_{2}-\frac{1}{\lambda^{2+p}} \int_{\mathbb{R}^{2}} \frac{\partial w}{\partial x_{1}} h\left(x+y_{0}\right) \psi_{2}\right] \\
& -\alpha_{k}^{2}\left[\frac{3}{a^{*}} \int_{\mathbb{R}^{2}} \frac{\partial w}{\partial x_{1}} w^{2} \psi_{1}+\frac{1}{\lambda^{2+p}} \int_{\mathbb{R}^{2}} \frac{\partial w}{\partial x_{1}} h\left(x+y_{0}\right) \psi_{1}-3 \int_{\mathbb{R}^{2}} \frac{\partial w}{\partial x_{1}} w \psi_{1}^{2}\right] \\
& +o\left(\left[\alpha_{k}+\beta_{k}\right]^{2}\right) .
\end{aligned}
$$

We claim that the coefficient $I_{3}$ of the term $\alpha_{k} \beta_{k}$ in (3.22) satisfies

$$
\begin{aligned}
I_{3}: & =\int_{\mathbb{R}^{2}} \frac{\partial w}{\partial x_{1}} \psi_{1}+6 \int_{\mathbb{R}^{2}} \frac{\partial w}{\partial x_{1}} w \psi_{1} \psi_{2}-\frac{1}{\lambda^{2+p}} \int_{\mathbb{R}^{2}} \frac{\partial w}{\partial x_{1}} h\left(x+y_{0}\right) \psi_{2} \\
& =\frac{1}{2 \lambda^{2+p}} \int_{\mathbb{R}^{2}} w\left[y_{0} \cdot \nabla h\left(x+y_{0}\right)\right] \frac{\partial w}{\partial x_{1}} .
\end{aligned}
$$

If (3.23) holds, we then derive from (3.22) that there exists some $x_{0}=\left(x_{10}, x_{20}\right) \in \mathbb{R}^{2}$ such that

$$
\begin{aligned}
& \frac{1}{2 \lambda^{2+p}} \int_{\mathbb{R}^{2}} \frac{\partial w^{2}}{\partial x_{j}}\left[\alpha_{k}\left(\frac{\lambda x_{k}}{\varepsilon_{k}}-y_{0}\right)-\alpha_{k} \beta_{k} \frac{y_{0}}{2}\right] \cdot \nabla h\left(x+y_{0}\right) \\
= & \alpha_{k}^{2} O\left(\left|x_{j 0}\right|\right)+o\left(\left[\alpha_{k}+\beta_{k}\right]^{2}\right), \quad j=1,2 .
\end{aligned}
$$

By the non-degeneracy assumption of (1.14), we further conclude from (3.24) that (3.15) holds for some $x_{0} \in \mathbb{R}^{2}$, and the lemma is therefore proved.

To complete the proof of the lemma, the rest is to prove the claim (3.23). Indeed, using the integration by parts, we derive from (3.10) that

$$
\begin{aligned}
A:= & \int_{\mathbb{R}^{2}} \frac{\partial w}{\partial x_{1}} \psi_{1}+6 \int_{\mathbb{R}^{2}} \frac{\partial w}{\partial x_{1}} w \psi_{1} \psi_{2} \\
= & \int_{\mathbb{R}^{2}} \frac{\partial w}{\partial x_{1}} \psi_{1}-3 \int_{\mathbb{R}^{2}} \frac{\partial w}{\partial x_{1}} w^{2} \psi_{1}-\frac{3}{2} \int_{\mathbb{R}^{2}} \frac{\partial w}{\partial x_{1}} \psi_{1}\left(x \cdot \nabla w^{2}\right) \\
= & \int_{\mathbb{R}^{2}} \frac{\partial w}{\partial x_{1}} \psi_{1}-3 \int_{\mathbb{R}^{2}} \frac{\partial w}{\partial x_{1}} w^{2} \psi_{1} \\
& +\frac{3}{2} \int_{\mathbb{R}^{2}} w^{2}\left[2 \frac{\partial w}{\partial x_{1}} \psi_{1}+x \cdot \nabla\left(\frac{\partial w}{\partial x_{1}} \psi_{1}\right)\right] \\
= & \int_{\mathbb{R}^{2}} \frac{\partial w}{\partial x_{1}} \psi_{1}+\frac{3}{2} \int_{\mathbb{R}^{2}} w^{2}\left[\frac{\partial w}{\partial x_{1}}\left(x \cdot \nabla \psi_{1}\right)+\psi_{1} x \cdot \nabla\left(\frac{\partial w}{\partial x_{1}}\right)\right] .
\end{aligned}
$$

Since $\left(x+y_{0}\right) \cdot \nabla h\left(x+y_{0}\right)=p h\left(x+y_{0}\right)$, we obtain from (1.14), (3.10) and (3.16) that

$$
\begin{aligned}
B: & =-\frac{1}{\lambda^{2+p}} \int_{\mathbb{R}^{2}} \frac{\partial w}{\partial x_{1}} h\left(x+y_{0}\right) \psi_{2} \\
& =\frac{1}{2 \lambda^{2+p}} \int_{\mathbb{R}^{2}} \frac{\partial w}{\partial x_{1}} h\left(x+y_{0}\right)(w+x \cdot \nabla w) \\
& =-\frac{1}{2 \lambda^{2+p}} \int_{\mathbb{R}^{2}} w\left[2 h\left(x+y_{0}\right) \frac{\partial w}{\partial x_{1}}+x \cdot \nabla\left(\frac{\partial w}{\partial x_{1}} h\left(x+y_{0}\right)\right)\right] \\
& =-\frac{1}{2 \lambda^{2+p}} \int_{\mathbb{R}^{2}} w\left\{\left[x \cdot \nabla h\left(x+y_{0}\right)\right] \frac{\partial w}{\partial x_{1}}+h\left(x+y_{0}\right) x \cdot \nabla\left(\frac{\partial w}{\partial x_{1}}\right)\right\} \\
& =-\frac{1}{2 \lambda^{2+p}} \int_{\mathbb{R}^{2}} w h\left(x+y_{0}\right)\left[x \cdot \nabla\left(\frac{\partial w}{\partial x_{1}}\right)\right]+\frac{1}{2 \lambda^{2+p}} \int_{\mathbb{R}^{2}} w\left[y_{0} \cdot \nabla h\left(x+y_{0}\right)\right] \frac{\partial w}{\partial x_{1}} .
\end{aligned}
$$


By above calculations, we then get from (3.23) that

$$
\begin{aligned}
I_{3}=A+B= & \int_{\mathbb{R}^{2}} \frac{\partial w}{\partial x_{1}} \psi_{1}+\frac{3}{2} \int_{\mathbb{R}^{2}} w^{2} \frac{\partial w}{\partial x_{1}}\left(x \cdot \nabla \psi_{1}\right) \\
& +\frac{1}{2} \int_{\mathbb{R}^{2}}\left[3 w^{2} \psi_{1}-\frac{w h\left(x+y_{0}\right)}{\lambda^{2+p}}\right]\left[x \cdot \nabla\left(\frac{\partial w}{\partial x_{1}}\right)\right] \\
& +\frac{1}{2 \lambda^{2+p}} \int_{\mathbb{R}^{2}} w\left[y_{0} \cdot \nabla h\left(x+y_{0}\right)\right] \frac{\partial w}{\partial x_{1}} \\
:= & I_{4}+\frac{1}{2 \lambda^{2+p}} \int_{\mathbb{R}^{2}} w\left[y_{0} \cdot \nabla h\left(x+y_{0}\right)\right] \frac{\partial w}{\partial x_{1}}
\end{aligned}
$$

Applying the integration by parts, we derive from (3.7) that

$$
\begin{aligned}
& \int_{\mathbb{R}^{2}} \frac{\partial w}{\partial x_{1}} \psi_{1}+\frac{1}{2} \int_{\mathbb{R}^{2}}\left[3 w^{2} \psi_{1}-\frac{w h\left(x+y_{0}\right)}{\lambda^{2+p}}\right]\left[x \cdot \nabla\left(\frac{\partial w}{\partial x_{1}}\right)\right] \\
= & \int_{\mathbb{R}^{2}} \frac{\partial w}{\partial x_{1}} \psi_{1}+\frac{1}{2} \int_{\mathbb{R}^{2}}\left[3 w^{2} \psi_{1}-\left(\frac{w^{3}}{a^{*}}+\frac{w h\left(x+y_{0}\right)}{\lambda^{2+p}}\right)\right]\left[x \cdot \nabla\left(\frac{\partial w}{\partial x_{1}}\right)\right] \\
= & \int_{\mathbb{R}^{2}} \frac{\partial w}{\partial x_{1}} \psi_{1}+\frac{1}{2} \int_{\mathbb{R}^{2}}\left(-\Delta \psi_{1}+\psi_{1}\right)\left[x \cdot \nabla\left(\frac{\partial w}{\partial x_{1}}\right)\right] \\
= & \int_{\mathbb{R}^{2}} \frac{\partial w}{\partial x_{1}} \psi_{1}+\frac{1}{2} \int_{\mathbb{R}^{2}}\left(-\Delta \psi_{1}\right)\left[x \cdot \nabla\left(\frac{\partial w}{\partial x_{1}}\right)\right]-\frac{1}{2} \int_{\mathbb{R}^{2}} \frac{\partial w}{\partial x_{1}}\left[2 \psi_{1}+x \cdot \nabla \psi_{1}\right] \\
= & \frac{1}{2} \int_{\mathbb{R}^{2}}\left(-\Delta \psi_{1}\right)\left[x \cdot \nabla\left(\frac{\partial w}{\partial x_{1}}\right)\right]-\frac{1}{2} \int_{\mathbb{R}^{2}} \frac{\partial w}{\partial x_{1}}\left(x \cdot \nabla \psi_{1}\right)
\end{aligned}
$$

which then gives from (3.25) that

$$
\begin{aligned}
-2 I_{4} & =\int_{\mathbb{R}^{2}} \Delta \psi_{1}\left[x \cdot \nabla\left(\frac{\partial w}{\partial x_{1}}\right)\right]+\int_{\mathbb{R}^{2}} \frac{\partial}{\partial x_{1}}\left[w-w^{3}\right]\left(x \cdot \nabla \psi_{1}\right) \\
& =\int_{\mathbb{R}^{2}} \Delta \psi_{1}\left[x \cdot \nabla\left(\frac{\partial w}{\partial x_{1}}\right)\right]+\int_{\mathbb{R}^{2}} \frac{\partial \Delta w}{\partial x_{1}}\left(x \cdot \nabla \psi_{1}\right) .
\end{aligned}
$$

To further simplify $I_{4}$, we next rewrite $\psi_{1}$ as $\psi_{1}(x)=\psi_{1}(r, \theta)$, where $x=r(\cos \theta, \sin \theta)$ 
and $(r, \theta)$ is the polar coordinate in $\mathbb{R}^{2}$. We then follow from (3.7) and (3.26) that

$$
\begin{aligned}
-2 I_{4}= & \int_{0}^{\infty} \int_{0}^{2 \pi}\left\{\left[r\left(\psi_{1}\right)_{r}\right]_{r}+\frac{1}{r}\left(\psi_{1}\right)_{\theta \theta}\right\} r \frac{\partial}{\partial r}\left(w^{\prime} \cos \theta\right) d \theta d r \\
& +\int_{0}^{\infty} \int_{0}^{2 \pi} \frac{\partial}{\partial r}\left(w^{\prime \prime}+\frac{w^{\prime}}{r}\right) \cos \theta r^{2}\left(\psi_{1}\right)_{r} d \theta d r \\
= & -\int_{0}^{\infty} \int_{0}^{2 \pi} r\left(\psi_{1}\right)_{r}\left(r w^{\prime \prime}\right)^{\prime} \cos \theta d \theta d r \\
& +\int_{0}^{\infty} \int_{0}^{2 \pi} r\left(\psi_{1}\right)_{r}\left[r \frac{\partial}{\partial r}\left(w^{\prime \prime}+\frac{w^{\prime}}{r}\right)\right] \cos \theta d \theta d r \\
& +\int_{0}^{\infty} \int_{0}^{2 \pi}\left(\psi_{1}\right)_{\theta \theta} w^{\prime \prime} \cos \theta d \theta d r \\
= & -\int_{0}^{\infty} \int_{0}^{2 \pi} r\left(\psi_{1}\right)_{r}\left\{\left(r w^{\prime \prime}\right)^{\prime}-\left[r \frac{\partial}{\partial r}\left(w^{\prime \prime}+\frac{w^{\prime}}{r}\right)\right]\right\} \cos \theta d \theta d r \\
& -\int_{0}^{\infty} \int_{0}^{2 \pi} \psi_{1} w^{\prime \prime} \cos \theta d \theta d r \\
= & -\int_{0}^{\infty} \int_{0}^{2 \pi}\left(\psi_{1}\right)_{r} w^{\prime} \cos \theta d \theta d r-\int_{0}^{\infty} \int_{0}^{2 \pi} \psi_{1} w^{\prime \prime} \cos \theta d \theta d r=0
\end{aligned}
$$

i.e., $I_{4}=0$, which therefore implies that the claim (3.23) holds by applying (3.25).

Remark 3.1. Whether the point $x_{0} \in \mathbb{R}^{2}$ in Lemma 3.2 is the origin or not is determined completely by the fact that whether the coefficient $I_{5}$ of the term $\alpha_{k}^{2}$ in (3.22) is zero or not, where $I_{5}$ satisfies

$$
I_{5}:=\frac{3}{a^{*}} \int_{\mathbb{R}^{2}} \frac{\partial w}{\partial x_{1}} w^{2} \psi_{1}+\frac{1}{\lambda^{2+p}} \int_{\mathbb{R}^{2}} \frac{\partial w}{\partial x_{1}} h\left(x+y_{0}\right) \psi_{1}-3 \int_{\mathbb{R}^{2}} \frac{\partial w}{\partial x_{1}} w \psi_{1}^{2} .
$$

If $h(x)$ is not even in $x$, it however seems difficult to derive that whether $I_{5}=0$ or not.

Lemma 3.3. Suppose that $V(x)=h(x) \in C^{2}\left(\mathbb{R}^{2}\right)$ satisfies $\lim _{|x| \rightarrow \infty} h(x)=\infty$ and (1.14) for some $y_{0} \in \mathbb{R}^{2}$, where $h(x)$ is homogeneous of degree $p \geq 2$. Then we have

$$
w_{k}:=\alpha_{k} \psi_{1}+\beta_{k} \psi_{2}+\alpha_{k}^{2} \psi_{3}+\beta_{k}^{2} \psi_{4}+\alpha_{k} \beta_{k} \psi_{5}+o\left(\left[\alpha_{k}+\beta_{k}\right]^{2}\right) \text { as } k \rightarrow \infty,
$$

where $\psi_{1}(x), \psi_{2}(x) \in C^{2}\left(\mathbb{R}^{2}\right) \cap L^{\infty}\left(\mathbb{R}^{2}\right)$ are given in Lemma 3.1 with $g(0)=1$, and $\psi_{i}(x) \in C^{2}\left(\mathbb{R}^{2}\right) \cap L^{\infty}\left(\mathbb{R}^{2}\right), i=3,4,5$, solves uniquely

$$
\nabla \psi_{i}(0)=0 \text { and } \mathcal{L} \psi_{i}(x)=f_{i}(x) \text { in } \mathbb{R}^{2}, \quad i=3,4,5,
$$

and $f_{i}(x)$ satisfies for some $y^{0} \in \mathbb{R}^{2}$,

$$
f_{i}(x)= \begin{cases}3 w \psi_{1}^{2}-\left(\frac{3 w^{2}}{a^{*}}+\frac{h\left(x+y_{0}\right)}{\lambda^{2+p}}\right) \psi_{1}-\frac{w}{\lambda^{1+p}}\left[y^{0} \cdot \nabla h\left(x+y_{0}\right)\right], & \text { if } i=3 \\ 3 w \psi_{2}^{2}+\psi_{2}, & \text { if } i=4 \\ 6 w \psi_{1} \psi_{2}+\psi_{1}-\left(\frac{3 w^{2}}{a^{*}}+\frac{h\left(x+y_{0}\right)}{\lambda^{2+p}}\right) \psi_{2} & \text { if } i=5 \\ -\frac{w}{2 \lambda^{2+p}}\left[y_{0} \cdot \nabla h\left(x+y_{0}\right)\right], & \end{cases}
$$

where $y_{0} \in \mathbb{R}^{2}$ is given by (1.14). Moreover, $\psi_{4}$ is radially symmetric. 
Proof. Following Lemma 3.1(3), set

$$
v_{k}=w_{k}-\alpha_{k} \psi_{1}-\beta_{k} \psi_{2}
$$

so that

$$
\begin{aligned}
\mathcal{L}_{k} w_{k} & =\mathcal{L}_{k}\left(v_{k}+\alpha_{k} \psi_{1}+\beta_{k} \psi_{2}\right) \\
& =\mathcal{L}_{k} v_{k}+\alpha_{k}\left(\mathcal{L}_{k}-\mathcal{L}\right) \psi_{1}+\beta_{k}\left(\mathcal{L}_{k}-\mathcal{L}\right) \psi_{2}+\alpha_{k} \mathcal{L} \psi_{1}+\beta_{k} \mathcal{L} \psi_{2} \\
& =\mathcal{L}_{k} v_{k}-w_{k}\left(\alpha_{k} \psi_{1}+\beta_{k} \psi_{2}\right)\left(3 w+w_{k}\right)-\alpha_{k}\left[\frac{w^{3}}{a^{*}}+\frac{h\left(x+y_{0}\right) w}{\lambda^{2+p}}\right]+\beta_{k} w .
\end{aligned}
$$

Applying (3.3), we then have

$$
\begin{aligned}
\mathcal{L}_{k} v_{k}= & \mathcal{L}_{k} w_{k}+w_{k}\left(\alpha_{k} \psi_{1}+\beta_{k} \psi_{2}\right)\left(3 w+w_{k}\right)+\alpha_{k}\left[\frac{w^{3}}{a^{*}}+\frac{h\left(x+y_{0}\right) w}{\lambda^{2+p}}\right]-\beta_{k} w \\
= & w_{k}\left(\alpha_{k} \psi_{1}+\beta_{k} \psi_{2}\right)\left(3 w+w_{k}\right)+\beta_{k}\left(\bar{u}_{k}-w\right) \\
& -\alpha_{k}\left\{\frac{1}{a^{*}}\left(\bar{u}_{k}^{3}-w^{3}\right)+\frac{1}{\lambda^{2+p}}\left[h\left(x+\frac{\lambda x_{k}}{\varepsilon_{k}}\right) \bar{u}_{k}-h\left(x+y_{0}\right) w\right]\right\} \\
= & w_{k}\left(\alpha_{k} \psi_{1}+\beta_{k} \psi_{2}\right)\left(3 w+w_{k}\right)+\beta_{k} w_{k}-I_{6},
\end{aligned}
$$

where $I_{6}$ satisfies

$$
\begin{aligned}
I_{6}= & \frac{\alpha_{k}}{a^{*}} w_{k}\left(3 w^{2}+3 w w_{k}+w_{k}^{2}\right) \\
& +\frac{\alpha_{k}}{\lambda^{2+p}}\left\{h\left(x+y_{0}\right)\left(\bar{u}_{k}-w\right)+\left[h\left(x+\frac{\lambda x_{k}}{\varepsilon_{k}}\right)-h\left(x+y_{0}\right)\right] \bar{u}_{k}\right\} \\
= & \frac{\alpha_{k}}{a^{*}} w_{k}\left(3 w^{2}+3 w w_{k}+w_{k}^{2}\right)+\frac{\alpha_{k}}{\lambda^{2+p}} h\left(x+y_{0}\right) w_{k} \\
& +\frac{\alpha_{k}}{\lambda^{2+p}}\left[\left(\frac{\lambda x_{k}}{\varepsilon_{k}}-y_{0}\right) \cdot \nabla h\left(x+y_{0}\right)\right] \bar{u}_{k}+o\left(\left[\alpha_{k}+\beta_{k}\right]^{2}\right) \\
= & \alpha_{k} w_{k}\left(\frac{3 w^{2}}{a^{*}}+\frac{h\left(x+y_{0}\right)}{\lambda^{2+p}}\right)+\frac{\alpha_{k}}{a^{*}} w_{k}^{2}\left(3 w+w_{k}\right) \\
& +\frac{\alpha_{k}}{\lambda^{2+p}}\left[\left(\frac{\lambda x_{k}}{\varepsilon_{k}}-y_{0}\right) \cdot \nabla h\left(x+y_{0}\right)\right] \bar{u}_{k}+o\left(\left[\alpha_{k}+\beta_{k}\right]^{2}\right),
\end{aligned}
$$

where Lemma 3.2 is used in the second equality. By Lemma 3.2 again, there exists $y^{0} \in \mathbb{R}^{2}$ such that

$$
\left|\alpha_{k}\left(\frac{\lambda x_{k}}{\varepsilon_{k}}-y_{0}\right)-\alpha_{k} \beta_{k} \frac{y_{0}}{2}-\alpha_{k}^{2} y^{0}\right|=o\left(\left[\alpha_{k}+\beta_{k}\right]^{2}\right) \text { as } k \rightarrow \infty .
$$

We thus obtain from above that

$$
\begin{aligned}
\mathcal{L}_{k} v_{k}= & w_{k}\left(\alpha_{k} \psi_{1}+\beta_{k} \psi_{2}\right)\left(3 w+w_{k}\right)+\beta_{k} w_{k}-\alpha_{k} w_{k}\left(\frac{3 w^{2}}{a^{*}}+\frac{h\left(x+y_{0}\right)}{\lambda^{2+p}}\right) \\
& -\frac{\alpha_{k}}{\lambda^{2+p}}\left[\left(\frac{\lambda x_{k}}{\varepsilon_{k}}-y_{0}\right) \cdot \nabla h\left(x+y_{0}\right)\right] \bar{u}_{k}-\frac{\alpha_{k}}{a^{*}} w_{k}^{2}\left(3 w+w_{k}\right)+o\left(\left[\alpha_{k}+\beta_{k}\right]^{2}\right) \\
= & \alpha_{k}^{2}\left\{3 w \psi_{1}^{2}-\left(\frac{3 w^{2}}{a^{*}}+\frac{h\left(x+y_{0}\right)}{\lambda^{2+p}}\right) \psi_{1}-\frac{w}{\lambda^{1+p}}\left[y^{0} \cdot \nabla h\left(x+y_{0}\right)\right]\right\} \\
& +\alpha_{k} \beta_{k}\left\{6 w \psi_{1} \psi_{2}+\psi_{1}-\left(\frac{3 w^{2}}{a^{*}}+\frac{h\left(x+y_{0}\right)}{\lambda^{2+p}}\right) \psi_{2}-\frac{1}{2 \lambda^{2+p}} w\left[y_{0} \cdot \nabla h\left(x+y_{0}\right)\right]\right\} \\
& +\beta_{k}^{2}\left(3 w \psi_{2}^{2}+\psi_{2}\right)+o\left(\left[\alpha_{k}+\beta_{k}\right]^{2}\right) \text { in } \mathbb{R}^{2} .
\end{aligned}
$$


Following (3.33), the same argument of proving Lemma 3.1 then gives (3.28). Finally, since $f_{4}(x)$ is radially symmetric, there exists a radial solution $\psi_{4}$. Further, the property (2.15) gives the uniqueness of $\psi_{4}$. Therefore, $\psi_{4}$ must be radially symmetric, and the proof is complete.

Lemma 3.4. Suppose that $V(x)=h(x) \in C^{2}\left(\mathbb{R}^{2}\right)$ satisfies $\lim _{|x| \rightarrow \infty} h(x)=\infty$ and (1.14) for some $y_{0} \in \mathbb{R}^{2}$, where $h(x)$ is homogeneous of degree $p \geq 2$. Then we have

$$
\int_{\mathbb{R}^{2}} w \psi_{1}=0, \quad \int_{\mathbb{R}^{2}} w \psi_{2}=0
$$

and

$$
I=\int_{\mathbb{R}^{2}}\left(2 w \psi_{4}+\psi_{2}^{2}\right)=0
$$

However, we have

$$
I I=2 \int_{\mathbb{R}^{2}} w \psi_{5}+2 \int_{\mathbb{R}^{2}} \psi_{1} \psi_{2}=-\frac{2+p}{2}<0 .
$$

Here $\psi_{1}(x), \cdots, \psi_{5}(x) \in C^{2}\left(\mathbb{R}^{2}\right) \cap L^{\infty}\left(\mathbb{R}^{2}\right)$ are given in Lemma 3.1 with $g(0)=1$ and Lemma 3.3 .

Since the proof of Lemma 3.4 is very involved, we leave it to the appendix. Following above lemmas, we are now ready to derive the comparison relation between $\beta_{k}$ and $\alpha_{k}$.

Proposition 3.5. Suppose that $V(x)=h(x) \in C^{2}\left(\mathbb{R}^{2}\right)$ satisfies $\lim _{|x| \rightarrow \infty} h(x)=\infty$ and (1.14) for some $y_{0} \in \mathbb{R}^{2}$, where $h(x)$ is homogeneous of degree $p \geq 2$. Then we have

$$
\beta_{k}=C^{*} \alpha_{k} \text { as } k \rightarrow \infty
$$

where the constant $C^{*}$ satisfies

$$
C^{*}=\frac{2}{2+p}\left(2 \int_{\mathbb{R}^{2}} w \psi_{3}+\int_{\mathbb{R}^{2}} \psi_{1}^{2}\right) \neq 0 .
$$

Moreover, $w_{k}$ satisfies

$$
w_{k}:=\left[\psi_{1}+C^{*} \psi_{2}\right] \alpha_{k}+\left[\psi_{3}+\left(C^{*}\right)^{2} \psi_{4}+C^{*} \psi_{5}\right] \alpha_{k}^{2}+o\left(\alpha_{k}^{2}\right) \text { as } k \rightarrow \infty,
$$

Here $\psi_{1}(x), \cdots, \psi_{5}(x) \in C^{2}\left(\mathbb{R}^{2}\right) \cap L^{\infty}\left(\mathbb{R}^{2}\right)$ are given in Lemma 3.1 with $g(0)=1$ and Lemma 3.3 .

Proof. Note from (3.2) that $w_{k}$ satisfies

$$
\int_{\mathbb{R}^{2}} w^{2}=\int_{\mathbb{R}^{2}} \bar{u}_{k}^{2}=\int_{\mathbb{R}^{2}}\left(w+w_{k}\right)^{2} \text {, i.e. } 2 \int_{\mathbb{R}^{2}} w w_{k}+\int_{\mathbb{R}^{2}} w_{k}^{2}=0 .
$$


Applying (3.40), we then derive from Lemma 3.3 that

$$
\begin{aligned}
0= & 2 \int_{\mathbb{R}^{2}} w w_{k}+\int_{\mathbb{R}^{2}} w_{k}^{2} \\
= & 2 \int_{\mathbb{R}^{2}} w\left(\alpha_{k} \psi_{1}+\beta_{k} \psi_{2}+\alpha_{k}^{2} \psi_{3}+\beta_{k}^{2} \psi_{4}+\alpha_{k} \beta_{k} \psi_{5}\right) \\
& +\int_{\mathbb{R}^{2}}\left(\alpha_{k} \psi_{1}+\beta_{k} \psi_{2}+\alpha_{k}^{2} \psi_{3}+\beta_{k}^{2} \psi_{4}+\alpha_{k} \beta_{k} \psi_{5}\right)^{2}+o\left(\left[\alpha_{k}+\beta_{k}\right]^{2}\right) \\
= & \alpha_{k}\left(2 \int_{\mathbb{R}^{2}} w \psi_{1}\right)+\beta_{k}\left(2 \int_{\mathbb{R}^{2}} w \psi_{2}\right)+\beta_{k}^{2}\left(2 \int_{\mathbb{R}^{2}} w \psi_{4}+\int_{\mathbb{R}^{2}} \psi_{2}^{2}\right) \\
& +\alpha_{k} \beta_{k}\left(2 \int_{\mathbb{R}^{2}} w \psi_{5}+2 \int_{\mathbb{R}^{2}} \psi_{1} \psi_{2}\right)+\alpha_{k}^{2}\left(2 \int_{\mathbb{R}^{2}} w \psi_{3}+\int_{\mathbb{R}^{2}} \psi_{1}^{2}\right)+o\left(\left[\alpha_{k}+\beta_{k}\right]^{2}\right) \\
= & -\frac{2+p}{2} \alpha_{k} \beta_{k}+\alpha_{k}^{2}\left(2 \int_{\mathbb{R}^{2}} w \psi_{3}+\int_{\mathbb{R}^{2}} \psi_{1}^{2}\right)+o\left(\left[\alpha_{k}+\beta_{k}\right]^{2}\right),
\end{aligned}
$$

where Lemma 3.4 is used in the last equality. It then follows from (3.41) that

$$
2 \int_{\mathbb{R}^{2}} w \psi_{3}+\int_{\mathbb{R}^{2}} \psi_{1}^{2} \neq 0
$$

and moreover,

$$
-\frac{2+p}{2} \beta_{k}+\alpha_{k}\left(2 \int_{\mathbb{R}^{2}} w \psi_{3}+\int_{\mathbb{R}^{2}} \psi_{1}^{2}\right)=0, \text { i.e., } \beta_{k}=C^{*} \alpha_{k},
$$

where $C^{*} \neq 0$ is as in (3.38). Finally, the expansion (3.39) follows directly from (3.37) and Lemma 3.3, and we are done.

We remark from (3.1) and Proposition 3.5 that the Lagrange multiplier $\mu_{k} \in \mathbb{R}$ of the Euler-Lagrange equation (2.16) satisfies

$$
\mu_{k}=-\frac{\lambda}{\varepsilon_{k}^{2}}+\lambda^{2} C^{*} \varepsilon_{k}^{p}+o\left(\varepsilon_{k}^{p}\right) \text { as } k \rightarrow \infty
$$

where $\lambda>0$ is defined by (2.2) with $g(0)=1$, and $C^{*} \neq 0$ is given by (3.38). Moreover, following above results we finally conclude the following refined spike profiles.

Theorem 3.6. Suppose that $V(x)=h(x) \in C^{2}\left(\mathbb{R}^{2}\right)$ satisfies $\lim _{|x| \rightarrow \infty} h(x)=\infty$ and (1.14) for some $y_{0} \in \mathbb{R}^{2}$, where $h(x)$ is homogeneous of degree $p \geq 2$. If $u_{a}$ is a positive minimizer of $e(a)$ for $a<a^{*}$. Then for any sequence $\left\{a_{k}\right\}$ with $a_{k} \nearrow a^{*}$ as $k \rightarrow \infty$, there exist a subsequence, still denoted by $\left\{a_{k}\right\}$, of $\left\{a_{k}\right\}$ and $\left\{x_{k}\right\} \subset \mathbb{R}^{2}$ such that the subsequence solution $u_{k}=u_{a_{k}}$ satisfies for $\varepsilon_{k}:=\left(a^{*}-a_{k}\right)^{\frac{1}{2+p}}$,

$$
\begin{aligned}
u_{k}(x)= & \frac{\lambda}{\|w\|_{2}}\left\{\frac{1}{\varepsilon_{k}} w\left(\frac{\lambda\left(x-x_{k}\right)}{\varepsilon_{k}}\right)+\varepsilon_{k}^{1+p}\left[\psi_{1}+C^{*} \psi_{2}\right]\left(\frac{\lambda\left(x-x_{k}\right)}{\varepsilon_{k}}\right)\right. \\
& \left.+\varepsilon_{k}^{3+2 p}\left[\psi_{3}+\left(C^{*}\right)^{2} \psi_{4}+C^{*} \psi_{5}\right]\left(\frac{\lambda\left(x-x_{k}\right)}{\varepsilon_{k}}\right)\right\}+o\left(\varepsilon_{k}^{3+2 p}\right) \text { as } k \rightarrow \infty
\end{aligned}
$$

uniformly in $\mathbb{R}^{2}$, where the unique maximum point $x_{k}$ of $u_{k}$ satisfies

$$
\left|\frac{\lambda x_{k}}{\varepsilon_{k}}-y_{0}\right|=\varepsilon_{k}^{2+p} O\left(\left|y^{0}\right|\right) \text { as } k \rightarrow \infty
$$

for some $y^{0} \in \mathbb{R}^{2}$, and $C^{*} \neq 0$ is given by (3.38). Here $\psi_{1}(x), \cdots, \psi_{5}(x) \in C^{2}\left(\mathbb{R}^{2}\right) \cap$ $L^{\infty}\left(\mathbb{R}^{2}\right)$ are given in Lemma 3.1 with $g(0)=1$ and Lemma 3.3. 
Proof. The refined spike profile (3.43) follows immediately from (3.2) and (3.39). Also, Lemma 3.2 and (3.37) yield that the estimate (3.44) holds.

Proof of Theorem 1.2. Since the local uniqueness of Theorem 1.1 implies that Theorem 3.6 holds for the whole sequence $\left\{a_{k}\right\}$, Theorem 1.2 is proved.

\section{$4 \quad$ Refined Spike Profiles: $V(x)=g(x) h(x)$}

The main purpose of this section is to derive Theorem 4.4 which extends the refined spike behavior of Theorem 1.2 to more general potentials $V(x)=g(x) h(x) \in C^{2}\left(\mathbb{R}^{2}\right)$, where $V(x)$ satisfies $\lim _{|x| \rightarrow \infty} V(x)=\infty$ and

$(V) . h(-x)=h(x)$ satisfies (1.14) and is homogeneous of degree $p \geq 2, g(x) \in C^{m}\left(\mathbb{R}^{2}\right)$ for some $2 \leq m \in \mathbb{N} \cup\{+\infty\}$ satisfies $0<C \leq g(x) \leq \frac{1}{C}$ in $\mathbb{R}^{2}$ and $G(x):=$ $g(x)-g(0)$

$$
\mathcal{D}^{\alpha} G(0)=0 \text { for all }|\alpha| \leq m-1, \text { and } \mathcal{D}^{\alpha} G(0) \neq 0 \text { for some }|\alpha|=m .
$$

Here it takes $m=+\infty$ if $g(x) \equiv 1$.

Remark 4.1. The property $h(-x)=h(x)$ in the above assumption $(V)$ implies that $y_{0}=0$ must occur in 1.14.

For the above type of potentials $V(x)$, suppose $\left\{u_{k}\right\}$ is a positive minimizer sequence of $e\left(a_{k}\right)$ with $a_{k} \nearrow a^{*}$ as $k \rightarrow \infty$, and let $w_{k}$ be defined by (3.2), where $x_{k}$ is the unique maximum point of $u_{k}$. Then Lemma 3.1 still holds in this case, where $\alpha_{k}>0$ and $\beta_{k}>0$ are defined in (3.1). Similar to Lemma 3.2, we start with the following estimates.

Lemma 4.1. Suppose $V(x)=g(x) h(x) \in C^{2}\left(\mathbb{R}^{2}\right)$ satisfies $\lim _{|x| \rightarrow \infty} V(x)=\infty$ and the assumption $(V)$ for $p \geq 2$ and $2 \leq m \in \mathbb{N} \cup\{+\infty\}$. Then the unique maximum point $x_{k}$ of $u_{k}$ satisfies the following estimates:

1. If $m$ is even, then we have

$$
\frac{\lambda \alpha_{k}\left|x_{k}\right|}{\varepsilon_{k}}=o\left(\left[\alpha_{k}+\beta_{k}\right]^{2}+\alpha_{k} \varepsilon_{k}^{m}\right) \text { as } k \rightarrow \infty
$$

2. If $m$ is odd, then we have

$$
\frac{\lambda \alpha_{k}\left|x_{k}\right|}{\varepsilon_{k}}=O\left(\alpha_{k} \varepsilon_{k}^{m}\left|x_{0}\right|\right)+o\left(\left[\alpha_{k}+\beta_{k}\right]^{2}+\alpha_{k} \varepsilon_{k}^{m}\right) \text { as } k \rightarrow \infty
$$

where $x_{0} \in \mathbb{R}^{2}$ satisfies

$$
g(0) \int_{\mathbb{R}^{2}} \frac{\partial w}{\partial x_{1}}\left[x_{0} \cdot \nabla h(x)\right] w+\frac{1}{\lambda^{m}} \sum_{|\alpha|=m} \int_{\mathbb{R}^{2}} \frac{\partial w}{\partial x_{1}}\left[\frac{x^{\alpha}}{\alpha !} \mathcal{D}^{\alpha} g(0)\right] h(x) w=0 .
$$

Proof. Recall that $\psi_{1}(x)$ and $\psi_{2}(x)$ are given in Lemma 3.1. Since $h(-x)=h(x)$, we have $\psi_{i}(-x)=\psi_{i}(x)$ for $i=1,2$ and thus

$$
\int_{\mathbb{R}^{2}} \frac{\partial w}{\partial x_{1}} \psi_{1}=\int_{\mathbb{R}^{2}} \frac{\partial w}{\partial x_{1}} w \psi_{1}^{2}=\int_{\mathbb{R}^{2}} \frac{\partial w}{\partial x_{1}} w \psi_{1} \psi_{2}=0
$$


Since (1.14) holds with $y_{0}=0$ as shown in Remark 4.1, the same calculations of (3.17)(3.18) then yield that

$$
\begin{aligned}
& o\left(\alpha_{k}^{2}+\alpha_{k} \beta_{k}\right)=\int_{\mathbb{R}^{2}} \frac{\partial w}{\partial x_{1}} \mathcal{L}_{k} w_{k} \\
= & o\left(\alpha_{k} \beta_{k}+\beta_{k}^{2}\right)-\alpha_{k} \int_{\mathbb{R}^{2}} \frac{\partial w}{\partial x_{1}}\left[\frac{1}{a^{*}} \bar{u}_{k}^{3}+\frac{\bar{u}_{k}}{\lambda^{2+p}} g\left(\frac{\varepsilon_{k} x}{\lambda}+x_{k}\right) h\left(x+\frac{\lambda x_{k}}{\varepsilon_{k}}\right)\right] \\
= & o\left(\alpha_{k} \beta_{k}+\beta_{k}^{2}\right)-\frac{\alpha_{k}}{a^{*}} \int_{\mathbb{R}^{2}} \frac{\partial w}{\partial x_{1}}\left(\bar{u}_{k}^{3}-w^{3}\right) \\
& -\frac{\alpha_{k}}{\lambda^{2+p}} \int_{\mathbb{R}^{2}} \frac{\partial w}{\partial x_{1}}\left[g\left(\frac{\varepsilon_{k} x}{\lambda}+x_{k}\right) h\left(x+\frac{\lambda x_{k}}{\varepsilon_{k}}\right) \bar{u}_{k}-g(0) h(x) w\right] \\
= & o\left(\alpha_{k} \beta_{k}+\beta_{k}^{2}\right)-\frac{\alpha_{k}}{\lambda^{2+p}} \int_{\mathbb{R}^{2}} \frac{\partial w}{\partial x_{1}}\left[g\left(\frac{\varepsilon_{k} x}{\lambda}+x_{k}\right) h\left(x+\frac{\lambda x_{k}}{\varepsilon_{k}}\right) \bar{u}_{k}-g(0) h(x) w\right] \\
= & o\left(\alpha_{k} \beta_{k}+\beta_{k}^{2}\right)-I_{1},
\end{aligned}
$$

where the first equality follows from (3.21) and (4.4). Similar to (3.19), we deduce from (1.14) with $y_{0}=0$ that

$$
\begin{aligned}
\frac{\lambda^{2+p}}{\alpha_{k}} I_{1}= & \int_{\mathbb{R}^{2}} \frac{\partial w}{\partial x_{1}}\left\{g(0) h\left(x+\frac{\lambda x_{k}}{\varepsilon_{k}}\right)\left[\bar{u}_{k}-w\right]+g(0)\left[h\left(x+\frac{\lambda x_{k}}{\varepsilon_{k}}\right)-h(x)\right] w\right\} \\
& +\int_{\mathbb{R}^{2}} \frac{\partial w}{\partial x_{1}}\left[g\left(\frac{\varepsilon_{k} x}{\lambda}+x_{k}\right)-g(0)\right] h\left(x+\frac{\lambda x_{k}}{\varepsilon_{k}}\right) \bar{u}_{k} \\
= & o\left(\alpha_{k}+\left|\frac{\lambda x_{k}}{\varepsilon_{k}}\right|+\beta_{k}\right)+g(0) \int_{\mathbb{R}^{2}} \frac{\partial w}{\partial x_{1}}\left(\frac{\lambda x_{k}}{\varepsilon_{k}} \cdot \nabla h(x)\right) w \\
& +\left(\frac{\varepsilon_{k}}{\lambda}\right)^{m} \sum_{|\alpha|=m} \int_{\mathbb{R}^{2}} \frac{\partial w}{\partial x_{1}}\left[\frac{1}{\alpha !}\left(x+\frac{\lambda x_{k}}{\varepsilon_{k}}\right)^{\alpha} \mathcal{D}^{\alpha} g(0)\right] h\left(x+\frac{\lambda x_{k}}{\varepsilon_{k}}\right) \bar{u}_{k}+o\left(\varepsilon_{k}^{m}\right),
\end{aligned}
$$

which then implies that

$$
\begin{aligned}
I_{1}= & \frac{\alpha_{k}}{\lambda^{2+p}}\left(\frac{\varepsilon_{k}}{\lambda}\right)^{m} \sum_{|\alpha|=m} \int_{\mathbb{R}^{2}} \frac{\partial w}{\partial x_{1}}\left[\frac{1}{\alpha !}\left(x+\frac{\lambda x_{k}}{\varepsilon_{k}}\right)^{\alpha} \mathcal{D}^{\alpha} g(0)\right] h\left(x+\frac{\lambda x_{k}}{\varepsilon_{k}}\right) \bar{u}_{k} \\
& +\frac{\alpha_{k}}{\lambda^{2+p}} g(0) \int_{\mathbb{R}^{2}} \frac{\partial w}{\partial x_{1}}\left(\frac{\lambda x_{k}}{\varepsilon_{k}} \cdot \nabla h(x)\right) w+o\left(\alpha_{k}^{2}+\alpha_{k} \beta_{k}+\left|\frac{\lambda x_{k}}{\varepsilon_{k}}\right|+\alpha_{k} \varepsilon_{k}^{m}\right) .
\end{aligned}
$$

Combining (4.5) and (4.6), we then conclude from the estimate (3.11) that

$$
\begin{aligned}
& \frac{\alpha_{k}}{\lambda^{2+p}} g(0) \int_{\mathbb{R}^{2}} \frac{\partial w}{\partial x_{1}}\left(\frac{\lambda x_{k}}{\varepsilon_{k}} \cdot \nabla h(x)\right) w \\
= & -\frac{\alpha_{k}}{\lambda^{2+p}}\left(\frac{\varepsilon_{k}}{\lambda}\right)^{m} \sum_{|\alpha|=m} \int_{\mathbb{R}^{2}} \frac{\partial w}{\partial x_{1}}\left[\frac{x^{\alpha}}{\alpha !} \mathcal{D}^{\alpha} g(0)\right] h(x) w+o\left(\left[\alpha_{k}+\beta_{k}\right]^{2}+\alpha_{k} \varepsilon_{k}^{m}\right) .
\end{aligned}
$$

If $m$ is even, one can note that

$$
\sum_{|\alpha|=m} \int_{\mathbb{R}^{2}} \frac{\partial w}{\partial x_{1}}\left[\frac{x^{\alpha}}{\alpha !} \mathcal{D}^{\alpha} g(0)\right] h(x) w=0,
$$

and it then follows from (4.7) and (1.14) with $y_{0}=0$ that (4.1) holds. If $m$ is odd, we then derive from (4.7) that both (4.2) and (4.3) hold. 
Lemma 4.2. Suppose $V(x)=g(x) h(x) \in C^{2}\left(\mathbb{R}^{2}\right)$ satisfies $\lim _{|x| \rightarrow \infty} V(x)=\infty$ and the assumption $(V)$ for $p \geq 2$ and $2 \leq m \in \mathbb{N} \cup\{+\infty\}$. Let $\psi_{1}(x)$ and $\psi_{2}(x)$ be given in Lemma 3.1 with $y_{0}=0$. Then $w_{k}$ satisfies

$$
\begin{aligned}
w_{k}:= & \alpha_{k} \psi_{1}+\beta_{k} \psi_{2}+\alpha_{k}^{2} \psi_{3}+\beta_{k}^{2} \psi_{4} \\
& +\alpha_{k} \varepsilon_{k}^{m} \phi+\alpha_{k} \beta_{k} \psi_{5}+o\left(\left[\alpha_{k}+\beta_{k}\right]^{2}+\alpha_{k} \varepsilon_{k}^{m}\right) \text { as } k \rightarrow \infty,
\end{aligned}
$$

where $\psi_{i}(x) \in C^{2}\left(\mathbb{R}^{2}\right) \cap L^{\infty}\left(\mathbb{R}^{2}\right), i=3,4,5$, solves uniquely

$$
\nabla \psi_{i}(0)=0 \text { and } \mathcal{L} \psi_{i}(x)=g_{i}(x) \text { in } \mathbb{R}^{2}, \quad i=3,4,5,
$$

and $g_{i}(x)$ satisfies

$$
g_{i}(x)= \begin{cases}3 w \psi_{1}^{2}-\left(\frac{3 w^{2}}{a^{*}}+\frac{g(0) h(x)}{\lambda^{2+p}}\right) \psi_{1}, & \text { if } i=3 ; \\ 3 w \psi_{2}^{2}+\psi_{2}, & \text { if } i=4 \\ 6 w \psi_{1} \psi_{2}+\psi_{1}-\left(\frac{3 w^{2}}{a^{*}}+\frac{g(0) h(x)}{\lambda^{2+p}}\right) \psi_{2}, & \text { if } i=5 .\end{cases}
$$

Here $\phi \in C^{2}\left(\mathbb{R}^{2}\right) \cap L^{\infty}\left(\mathbb{R}^{2}\right)$ solves uniquely

$$
\begin{aligned}
\mathcal{L} \phi(x)=-\frac{1}{\lambda^{2+p}}\{ & {\left[x_{0} \cdot \nabla h(x)\right] g(0) w } \\
& \left.+\frac{1}{\lambda^{m}} \sum_{|\alpha|=m}\left[\frac{x^{\alpha}}{\alpha !} \mathcal{D}^{\alpha} g(0)\right] h(x) w\right\} \text { in } \mathbb{R}^{2}, \text { and } \nabla \phi(0)=0,
\end{aligned}
$$

where $x_{0}=0$ holds for the case where $m$ is even, and $x_{0} \in \mathbb{R}^{2}$ satisfies (4.3) for the case where $m$ is odd.

Proof. Following Lemma 3.1(3), we set

$$
v_{k}=w_{k}-\alpha_{k} \psi_{1}-\beta_{k} \psi_{2} .
$$

Similar to (3.32), we then have

$$
\begin{aligned}
\mathcal{L}_{k} v_{k}= & w_{k}\left(\alpha_{k} \psi_{1}+\beta_{k} \psi_{2}\right)\left(3 w+w_{k}\right)+\beta_{k} w_{k}-\frac{\alpha_{k}}{a^{*}}\left(\bar{u}_{k}^{3}-w^{3}\right) \\
& -\frac{\alpha_{k}}{\lambda^{2+p}}\left[g\left(\frac{\varepsilon_{k} x}{\lambda}+x_{k}\right) h\left(x+\frac{\lambda x_{k}}{\varepsilon_{k}}\right) \bar{u}_{k}-g(0) h(x) w\right] \\
= & w_{k}\left(\alpha_{k} \psi_{1}+\beta_{k} \psi_{2}\right)\left(3 w+w_{k}\right)+\beta_{k} w_{k} \\
& -\frac{\alpha_{k}}{a^{*}} w_{k}\left(3 w^{2}+3 w w_{k}+w_{k}^{2}\right)-I_{2},
\end{aligned}
$$


where $I_{2}$ satisfies

$$
\begin{aligned}
& I_{2}=\frac{\alpha_{k}}{\lambda^{2+p}}\{ {\left[g\left(\frac{\varepsilon_{k} x}{\lambda}+x_{k}\right)-g(0)\right] h\left(x+\frac{\lambda x_{k}}{\varepsilon_{k}}\right) \bar{u}_{k} } \\
&\left.+g(0)\left[h\left(x+\frac{\lambda x_{k}}{\varepsilon_{k}}\right)-h(x)\right] \bar{u}_{k}+g(0) h(x)\left(\bar{u}_{k}-w\right)\right\} \\
&=\frac{\alpha_{k}}{\lambda^{2+p}}\left\{\left(\frac{\varepsilon_{k}}{\lambda}\right)^{m} \sum_{|\alpha|=m}\left[\frac{1}{\alpha !}\left(x+\frac{\lambda x_{k}}{\varepsilon_{k}}\right)^{\alpha} \mathcal{D}^{\alpha} g(0)\right] h\left(x+\frac{\lambda x_{k}}{\varepsilon_{k}}\right) \bar{u}_{k}\right. \\
&\left.\quad+g(0)\left(\frac{\lambda x_{k}}{\varepsilon_{k}} \cdot \nabla h(x)\right) \bar{u}_{k}+g(0) h(x) w_{k}\right\}+o\left(\frac{\alpha_{k} x_{k}}{\varepsilon_{k}}+\alpha_{k} \varepsilon_{k}^{m}\right) \\
&=\alpha_{k} w_{k} \frac{g(0) h(x)}{\lambda^{2+p}}+\frac{\alpha_{k}}{\lambda^{2+p}}\left(\frac{\lambda x_{k}}{\varepsilon_{k}} \cdot \nabla h(x)\right) g(0) \bar{u}_{k} \\
& \quad+\frac{\alpha_{k} \varepsilon_{k}^{m}}{\lambda^{2+p+m}} \sum_{|\alpha|=m}\left[\frac{1}{\alpha !}\left(x+\frac{\lambda x_{k}}{\varepsilon_{k}}\right)^{\alpha} \mathcal{D}^{\alpha} g(0)\right] h\left(x+\frac{\lambda x_{k}}{\varepsilon_{k}}\right) \bar{u}_{k}+o\left(\alpha_{k} \varepsilon_{k}^{m}\right),
\end{aligned}
$$

where Lemma 4.1 is used in the last equality. Applying Lemma 4.1 again, we then obtain from (4.12) and (4.13) that

$$
\begin{aligned}
& \mathcal{L}_{k} v_{k}=w_{k}\left(\alpha_{k} \psi_{1}+\beta_{k} \psi_{2}\right)\left(3 w+w_{k}\right)+\beta_{k} w_{k}-\frac{\alpha_{k}}{a^{*}} w_{k}^{2}\left(3 w+w_{k}\right) \\
& -\alpha_{k} w_{k}\left[\frac{3 w^{2}}{a^{*}}+\frac{g(0) h(x)}{\lambda^{2+p}}\right]-\frac{\alpha_{k}}{\lambda^{2+p}}\left(\frac{\lambda x_{k}}{\varepsilon_{k}} \cdot \nabla h(x)\right) g(0) \bar{u}_{k} \\
& -\frac{\alpha_{k} \varepsilon_{k}^{m}}{\lambda^{2+p+m}} \sum_{|\alpha|=m}\left[\frac{1}{\alpha !}\left(x+\frac{\lambda x_{k}}{\varepsilon_{k}}\right)^{\alpha} \mathcal{D}^{\alpha} g(0)\right] h\left(x+\frac{\lambda x_{k}}{\varepsilon_{k}}\right) \bar{u}_{k}+o\left(\alpha_{k} \varepsilon_{k}^{m}\right) \\
& =\alpha_{k}^{2}\left[3 w \psi_{1}^{2}-\left(\frac{3 w^{2}}{a^{*}}+\frac{g(0) h(x)}{\lambda^{2+p}}\right) \psi_{1}\right] \\
& +\alpha_{k} \beta_{k}\left[6 w \psi_{1} \psi_{2}+\psi_{1}-\left(\frac{3 w^{2}}{a^{*}}+\frac{g(0) h(x)}{\lambda^{2+p}}\right) \psi_{2}\right] \\
& -\frac{\alpha_{k} \varepsilon_{k}^{m}}{\lambda^{2+p}}\left\{\left[x_{0} \cdot \nabla h(x)\right] g(0) w+\frac{1}{\lambda^{m}} \sum_{|\alpha|=m}\left[\frac{x^{\alpha}}{\alpha !} \mathcal{D}^{\alpha} g(0)\right] h(x) w\right\} \\
& +\beta_{k}^{2}\left(3 w \psi_{2}^{2}+\psi_{2}\right)+o\left(\left[\alpha_{k}+\beta_{k}\right]^{2}+\alpha_{k} \varepsilon_{k}^{m}\right) \text { in } \mathbb{R}^{2},
\end{aligned}
$$

where $x_{0}=0$ holds for the case where $m$ is even, and $x_{0} \in \mathbb{R}^{2}$ satisfies (4.3) for the case where $m$ is odd. Following (4.14), the same argument of proving Lemma 3.1 then gives (4.8), and the proof is therefore complete.

Proposition 4.3. Suppose $V(x)=g(x) h(x) \in C^{2}\left(\mathbb{R}^{2}\right)$ satisfies $\lim _{|x| \rightarrow \infty} V(x)=\infty$ and the assumption $(V)$ for $p \geq 2$ and $2 \leq m \in \mathbb{N} \cup\{+\infty\}$. Let $\psi_{1}(x), \cdots, \psi_{5}(x) \in$ $C^{2}\left(\mathbb{R}^{2}\right) \cap L^{\infty}\left(\mathbb{R}^{2}\right)$ be given in Lemma 3.1 with $y_{0}=0$ and Lemma 4.2. and $\phi$ is given by (4.11).

1. If $m>2+p$, then

$$
\beta_{k}=C^{*} \alpha_{k}
$$

and $w_{k}$ satisfies

$$
w_{k}:=\left[\psi_{1}+C^{*} \psi_{2}\right] \alpha_{k}+\left[\psi_{3}+\left(C^{*}\right)^{2} \psi_{4}+C^{*} \psi_{5}\right] \alpha_{k}^{2}+o\left(\alpha_{k}^{2}\right) \text { as } k \rightarrow \infty,
$$

where the constant $C^{*}$ satisfies

$$
C^{*}:=\frac{2}{2+p}\left(2 \int_{\mathbb{R}^{2}} w \psi_{3}+\int_{\mathbb{R}^{2}} \psi_{1}^{2}\right) \neq 0
$$


2. If $1 \leq m \leq 2+p$ and $m$ is odd, then $\beta_{k}=C^{*} \alpha_{k}$ and $w_{k}$ satisfies

$$
\begin{aligned}
w_{k}:= & {\left[\psi_{1}+C^{*} \psi_{2}\right] \alpha_{k}+\phi \alpha_{k} \varepsilon_{k}^{m} } \\
& +\left[\psi_{3}+\left(C^{*}\right)^{2} \psi_{4}+C^{*} \psi_{5}\right] \alpha_{k}^{2}+o\left(\alpha_{k} \varepsilon_{k}^{m}\right) \text { as } k \rightarrow \infty,
\end{aligned}
$$

where the constant $C^{*} \neq 0$ is given by (4.17).

3. If $1 \leq m<2+p$ and $m$ is even, consider

$$
\mathcal{S}=\sum_{|\alpha|=m} \int_{\mathbb{R}^{2}}\left[\frac{x^{\alpha}}{\alpha !} \mathcal{D}^{\alpha} g(0)\right] h(x) w^{2} .
$$

Then for the case where $\mathcal{S}=0$, we have $\beta_{k}=C^{*} \alpha_{k}$ and $w_{k}$ satisfies (4.18), where the constant $C^{*} \neq 0$ is given by (4.17). However, for the case where $\mathcal{S} \neq 0$, we have

$$
\beta_{k}=C_{1}^{*} \varepsilon_{k}^{m}
$$

and $w_{k}$ satisfies

$$
w_{k}:=C_{1}^{*} \psi_{2} \varepsilon_{k}^{m}+\psi_{1} \alpha_{k}+\left(C_{1}^{*}\right)^{2} \psi_{4} \varepsilon_{k}^{2 m}+o\left(\varepsilon_{k}^{\min \{2+p, 2 m\}}\right) \text { as } k \rightarrow \infty,
$$

where the constant $C_{1}^{*}$ satisfies

$$
C_{1}^{*}=-\frac{m+p}{(2+p) \lambda^{2+p+m}} \sum_{|\alpha|=m} \int_{\mathbb{R}^{2}}\left[\frac{x^{\alpha}}{\alpha !} \mathcal{D}^{\alpha} g(0)\right] h(x) w^{2} \neq 0 .
$$

4. If $m=2+p$ is even, then

$$
\beta_{k}=C_{2}^{*} \alpha_{k}
$$

and $w_{k}$ satisfies

$$
w_{k}:=\left[\psi_{1}+C_{2}^{*} \psi_{2}\right] \alpha_{k}+\left[\psi_{3}+\left(C_{2}^{*}\right)^{2} \psi_{4}+C_{2}^{*} \psi_{5}+\phi\right] \alpha_{k}^{2}+o\left(\alpha_{k}^{2}\right) \text { as } k \rightarrow \infty,
$$

where the constant $C_{2}^{*}$ satisfies

$$
C_{2}^{*}=\frac{2}{2+p}\left[2 \int_{\mathbb{R}^{2}} w \psi_{3}+\int_{\mathbb{R}^{2}} \psi_{1}^{2}+2 \int_{\mathbb{R}^{2}} w \phi\right] \neq 0 .
$$

Proof. The same argument of proving Lemma 3.4 with $y_{0}=0$ yields that

$$
\int_{\mathbb{R}^{2}} w \psi_{1}=0, \quad \int_{\mathbb{R}^{2}} w \psi_{2}=0 \text { and } I=\int_{\mathbb{R}^{2}}\left(2 w \psi_{4}+\psi_{2}^{2}\right)=0
$$

and

$$
I I=2 \int_{\mathbb{R}^{2}} w \psi_{5}+2 \int_{\mathbb{R}^{2}} \psi_{1} \psi_{2}=-\frac{2+p}{2}<0 .
$$


It thus follows from (3.40) and Lemma 4.2 that

$$
\begin{aligned}
0= & 2 \int_{\mathbb{R}^{2}} w w_{k}+\int_{\mathbb{R}^{2}} w_{k}^{2} \\
= & 2 \int_{\mathbb{R}^{2}} w\left(\alpha_{k} \psi_{1}+\beta_{k} \psi_{2}+\alpha_{k}^{2} \psi_{3}+\beta_{k}^{2} \psi_{4}+\alpha_{k} \varepsilon_{k}^{m} \phi+\alpha_{k} \beta_{k} \psi_{5}\right) \\
& +\int_{\mathbb{R}^{2}}\left(\alpha_{k} \psi_{1}+\beta_{k} \psi_{2}+\alpha_{k}^{2} \psi_{3}+\beta_{k}^{2} \psi_{4}+\alpha_{k} \varepsilon_{k}^{m} \phi+\alpha_{k} \beta_{k} \psi_{5}\right)^{2} \\
& +o\left(\left[\alpha_{k}+\beta_{k}\right]^{2}+\alpha_{k} \varepsilon_{k}^{m}\right) \\
= & \alpha_{k}\left(2 \int_{\mathbb{R}^{2}} w \psi_{1}\right)+\beta_{k}\left(2 \int_{\mathbb{R}^{2}} w \psi_{2}\right)+\beta_{k}^{2}\left(2 \int_{\mathbb{R}^{2}} w \psi_{4}+\int_{\mathbb{R}^{2}} \psi_{2}^{2}\right) \\
& +\alpha_{k} \beta_{k}\left(2 \int_{\mathbb{R}^{2}} w \psi_{5}+2 \int_{\mathbb{R}^{2}} \psi_{1} \psi_{2}\right)+\alpha_{k}^{2}\left(2 \int_{\mathbb{R}^{2}} w \psi_{3}+\int_{\mathbb{R}^{2}} \psi_{1}^{2}\right) \\
& +\alpha_{k} \varepsilon_{k}^{m}\left(2 \int_{\mathbb{R}^{2}} w \phi\right)+o\left(\left[\alpha_{k}+\beta_{k}\right]^{2}+\alpha_{k} \varepsilon_{k}^{m}\right) \\
= & -\frac{2+p}{2} \alpha_{k} \beta_{k}+\alpha_{k}^{2}\left(2 \int_{\mathbb{R}^{2}} w \psi_{3}+\int_{\mathbb{R}^{2}} \psi_{1}^{2}\right)+\alpha_{k} \varepsilon_{k}^{m}\left(2 \int_{\mathbb{R}^{2}} w \phi\right) \\
& +o\left(\left[\alpha_{k}+\beta_{k}\right]^{2}+\alpha_{k} \varepsilon_{k}^{m}\right),
\end{aligned}
$$

where (4.26) and (4.27) are used in the last equality. Following (4.28), we next carry out the proof by considering separately the following four cases:

Case 1. $m>2+p$. In this case, it follows from (4.28) that the constant $C^{*}$ defined in (4.17) is nonzero and

$$
-\frac{2+p}{2} \beta_{k}+\alpha_{k}\left(2 \int_{\mathbb{R}^{2}} w \psi_{3}+\int_{\mathbb{R}^{2}} \psi_{1}^{2}\right)=0, \text { i.e., } \beta_{k}=C^{*} \alpha_{k}
$$

Moreover, the expansion (4.16) follows directly from (4.15) and Lemma 4.2, and Case 1 is therefore proved.

Case 2. $1 \leq m \leq 2+p$ and $m$ is odd. In this case, since $m$ is odd and $h(-x)=h(x)$, we obtain from (3.10) and (4.11) that

$$
\begin{aligned}
2 \int_{\mathbb{R}^{2}} w \phi= & 2 \int_{\mathbb{R}^{2}} \phi \mathcal{L} \psi_{2}=2 \int_{\mathbb{R}^{2}} \psi_{2} \mathcal{L} \phi \\
= & \frac{1}{\lambda^{2+p}} \int_{\mathbb{R}^{2}}\left\{\left[x_{0} \cdot \nabla h(x)\right] g(0) w\right. \\
& \left.+\frac{1}{\lambda^{m}} \sum_{|\alpha|=m}\left[\frac{x^{\alpha}}{\alpha !} \mathcal{D}^{\alpha} g(0)\right] h(x) w\right\}(w+x \cdot \nabla w)=0
\end{aligned}
$$

We then derive from (4.28) that (4.17) still holds and thus $\beta_{k}=C^{*} \alpha_{k}$. Further, the expansion (4.18) follows directly from (4.8) and (4.15).

Case 3. $1 \leq m<2+p$ and $m$ is even. Since $m$ is even, then $x_{0}=0$ holds in (4.11). 
Further, since $x^{\alpha} h(x)$ is homogeneous of degree $m+p$, we then obtain from (4.11) that

$$
\begin{aligned}
2 \int_{\mathbb{R}^{2}} w \phi= & 2 \int_{\mathbb{R}^{2}} \phi \mathcal{L} \psi_{2}=2 \int_{\mathbb{R}^{2}} \psi_{2} \mathcal{L} \phi \\
= & \frac{1}{\lambda^{2+p+m}} \sum_{|\alpha|=m} \int_{\mathbb{R}^{2}}\left[\frac{x^{\alpha}}{\alpha !} \mathcal{D}^{\alpha} g(0)\right] h(x) w(w+x \cdot \nabla w) \\
= & \frac{1}{\lambda^{2+p+m}} \sum_{|\alpha|=m} \int_{\mathbb{R}^{2}}\left[\frac{x^{\alpha}}{\alpha !} \mathcal{D}^{\alpha} g(0)\right] h(x) w^{2} \\
& +\frac{1}{2 \lambda^{2+p+m}} \sum_{|\alpha|=m} \int_{\mathbb{R}^{2}}\left[\frac{x^{\alpha}}{\alpha !} \mathcal{D}^{\alpha} g(0)\right] h(x)\left(x \cdot \nabla w^{2}\right) \\
= & \frac{1}{\lambda^{2+p+m}} \sum_{|\alpha|=m} \int_{\mathbb{R}^{2}}\left[\frac{x^{\alpha}}{\alpha !} \mathcal{D}^{\alpha} g(0)\right] h(x) w^{2} \\
& -\frac{1}{2 \lambda^{2+p+m}} \sum_{|\alpha|=m} \int_{\mathbb{R}^{2}} w^{2}\left\{2\left[\frac{x^{\alpha}}{\alpha !} \mathcal{D}^{\alpha} g(0) h(x)\right]\right. \\
& \left.+x \cdot \nabla\left[\frac{x^{\alpha}}{\alpha !} \mathcal{D}^{\alpha} g(0) h(x)\right]\right\} \\
= & -\frac{m+p}{2 \lambda^{2+p+m}} \sum_{|\alpha|=m} \int_{\mathbb{R}^{2}}\left[\frac{x^{\alpha}}{\alpha !} \mathcal{D}^{\alpha} g(0)\right] h(x) w^{2}:=-\frac{m+p}{2 \lambda^{2+p+m}} \mathcal{S},
\end{aligned}
$$

where $\mathcal{S}$ is as in (4.19). Therefore, if $\mathcal{S}=0$, then we are in the same situation as that of above Case 2, which gives that $\beta_{k}=C^{*} \alpha_{k}$ and $w_{k}$ satisfies (4.18), where the constant $C^{*} \neq 0$ is given by (4.17).

We next consider the case where $\mathcal{S} \neq 0$. By applying (4.29), in this case we derive from (4.28) that

$$
-\frac{2+p}{2} \alpha_{k} \beta_{k}+\alpha_{k} \varepsilon_{k}^{m}\left(2 \int_{\mathbb{R}^{2}} w \phi\right)=0
$$

which implies that $\beta_{k}=C_{1}^{*} \varepsilon_{k}^{m}$, where the constant $C_{1}^{*} \neq 0$ satisfies (4.22) in view of (4.29). Further, the expansion (4.21) follows directly from (4.20) and Lemma 4.2.

Case 4. $m=2+p$ is even. In this case, we derive from (4.28) that

$$
-\frac{2+p}{2} \alpha_{k} \beta_{k}+\alpha_{k}^{2}\left(2 \int_{\mathbb{R}^{2}} w \psi_{3}+\int_{\mathbb{R}^{2}} \psi_{1}^{2}+2 \int_{\mathbb{R}^{2}} w \phi\right)=0
$$

which gives that $\beta_{k}=C_{2}^{*} \alpha_{k}$, where the constant $C_{2}^{*} \neq 0$ satisfies (4.25). Further, the expansion (4.24) follows directly from (4.23) and Lemma 4.2.

Applying directly Lemmas 4.1 and 4.2 as well as Proposition 4.3, we now conclude the following main results of this section. Recall that $\lambda>0$ is defined by (2.2) with $y_{0}=0, \psi_{1}(x), \cdots, \psi_{5}(x) \in C^{2}\left(\mathbb{R}^{2}\right) \cap L^{\infty}\left(\mathbb{R}^{2}\right)$ are given in Lemma 3.1 with $y_{0}=0$ and Lemma 4.2, and $\phi$ is given by (4.11).

Theorem 4.4. Suppose $V(x)=g(x) h(x) \in C^{2}\left(\mathbb{R}^{2}\right)$ satisfies $\lim _{|x| \rightarrow \infty} V(x)=\infty$ and the assumption $(V)$ for $p \geq 2$ and $2 \leq m \in \mathbb{N} \cup\{+\infty\}$. Let $u_{a}$ be a positive minimizer of (1.1) for $a<a^{*}$. Then for any sequence $\left\{a_{k}\right\}$ with $a_{k} \nearrow a^{*}$ as $k \rightarrow \infty$, there exists a subsequence, still denoted by $\left\{a_{k}\right\}$, of $\left\{a_{k}\right\}$ such that $u_{k}=u_{a_{k}}$ has a unique maximum point $x_{k} \in \mathbb{R}^{2}$ and satisfies for $\varepsilon_{k}:=\left(a^{*}-a_{k}\right)^{\frac{1}{2+p}}$, 
1. If $m>2+p$, then we have

$$
\begin{aligned}
u_{k}(x)= & \frac{\lambda}{\|w\|_{2}}\left\{\frac{1}{\varepsilon_{k}} w\left(\frac{\lambda\left(x-x_{k}\right)}{\varepsilon_{k}}\right)+\varepsilon_{k}^{1+p}\left[\psi_{1}+C^{*} \psi_{2}\right]\left(\frac{\lambda\left(x-x_{k}\right)}{\varepsilon_{k}}\right)\right. \\
& \left.+\varepsilon_{k}^{3+2 p}\left[\psi_{3}+\left(C^{*}\right)^{2} \psi_{4}+C^{*} \psi_{5}\right]\left(\frac{\lambda\left(x-x_{k}\right)}{\varepsilon_{k}}\right)\right\}+o\left(\varepsilon_{k}^{3+2 p}\right) \text { as } k \rightarrow \infty
\end{aligned}
$$

uniformly in $\mathbb{R}^{2}$, where $x_{k}$ satisfies

$$
\frac{\left|x_{k}\right|}{\varepsilon_{k}}=O\left(\varepsilon_{k}^{m}\left|y^{0}\right|\right)+o\left(\varepsilon_{k}^{2+p}\right) \text { as } k \rightarrow \infty
$$

for some $y^{0} \in \mathbb{R}^{2}$, and the constant $C^{*} \neq 0$ is given by (4.17). Further, if $m$ is even, then $x_{k}$ satisfies

$$
\frac{\left|x_{k}\right|}{\varepsilon_{k}^{3+p}}=o(1) \text { as } k \rightarrow \infty .
$$

2. If $1 \leq m \leq 2+p$ and $m$ is odd, then we have

$$
\begin{aligned}
u_{k}(x)=\frac{\lambda}{\|w\|_{2}}\{ & \frac{1}{\varepsilon_{k}} w\left(\frac{\lambda\left(x-x_{k}\right)}{\varepsilon_{k}}\right)+\varepsilon_{k}^{1+p}\left[\psi_{1}+C^{*} \psi_{2}\right]\left(\frac{\lambda\left(x-x_{k}\right)}{\varepsilon_{k}}\right) \\
& +\varepsilon_{k}^{3+2 p}\left[\psi_{3}+\left(C^{*}\right)^{2} \psi_{4}+C^{*} \psi_{5}\right]\left(\frac{\lambda\left(x-x_{k}\right)}{\varepsilon_{k}}\right) \\
& \left.+\varepsilon_{k}^{1+m+p} \phi\left(\frac{\lambda\left(x-x_{k}\right)}{\varepsilon_{k}}\right)\right\}+o\left(\varepsilon_{k}^{1+m+p}\right) \text { as } k \rightarrow \infty
\end{aligned}
$$

uniformly in $\mathbb{R}^{2}$, where $x_{k}$ satisfies

$$
\frac{\left|x_{k}\right|}{\varepsilon_{k}^{m+1}}=O\left(\left|y^{0}\right|\right) \text { as } k \rightarrow \infty .
$$

for some $y^{0} \in \mathbb{R}^{2}$, and the constant $C^{*} \neq 0$ is given by (4.17).

3. If $m=2+p$ is even, then we have

$$
\begin{aligned}
u_{k}(x)= & \frac{\lambda}{\|w\|_{2}}\left\{\frac{1}{\varepsilon_{k}} w\left(\frac{\lambda\left(x-x_{k}\right)}{\varepsilon_{k}}\right)+\varepsilon_{k}^{1+p}\left[\psi_{1}+C_{2}^{*} \psi_{2}\right]\left(\frac{\lambda\left(x-x_{k}\right)}{\varepsilon_{k}}\right)\right. \\
& \left.+\varepsilon_{k}^{3+2 p}\left[\psi_{3}+\left(C_{2}^{*}\right)^{2} \psi_{4}+C_{2}^{*} \psi_{5}+\phi\right]\left(\frac{\lambda\left(x-x_{k}\right)}{\varepsilon_{k}}\right)\right\}+o\left(\varepsilon_{k}^{3+2 p}\right) \text { as } k \rightarrow \infty
\end{aligned}
$$

uniformly in $\mathbb{R}^{2}$, where $x_{k}$ satisfies (4.32) and the constant $C_{2}^{*} \neq 0$ is defined by (4.25).

4. If $1 \leq m<2+p$ and $m$ is even, let the constant $\mathcal{S}$ be defined in (4.19). Then for the case where $\mathcal{S}=0, u_{k}$ satisfies (4.33) and $x_{k}$ satisfies

$$
\frac{\left|x_{k}\right|}{\varepsilon_{k}^{m+1}}=o(1) \text { as } k \rightarrow \infty \text {. }
$$

However, for the case where $\mathcal{S} \neq 0, u_{k}$ satisfies

$$
\begin{aligned}
u_{k}(x)= & \frac{\lambda}{\|w\|_{2}}\left\{\frac{1}{\varepsilon_{k}} w\left(\frac{\lambda\left(x-x_{k}\right)}{\varepsilon_{k}}\right)+\varepsilon_{k}^{m-1} C_{1}^{*} \psi_{2}\left(\frac{\lambda\left(x-x_{k}\right)}{\varepsilon_{k}}\right)\right. \\
& \left.+\varepsilon_{k}^{2 m-1}\left(C_{1}^{*}\right)^{2} \psi_{4}\left(\frac{\lambda\left(x-x_{k}\right)}{\varepsilon_{k}}\right)+\varepsilon_{k}^{1+p} \psi_{1}\left(\frac{\lambda\left(x-x_{k}\right)}{\varepsilon_{k}}\right)\right\} \\
& +o\left(\varepsilon_{k}^{\min \{2+p, 2 m\}-1}\right) \text { as } k \rightarrow \infty
\end{aligned}
$$


uniformly in $\mathbb{R}^{2}$, where $x_{k}$ satisfies $(4.36)$, and the constant $C_{1}^{*} \neq 0$ is defined by (4.22).

Proof. (1). If $m>2+p$, then (4.30) follows directly from Proposition 4.3(1), and (4.31) follows from Lemma 4.1. Specially, if $m$ is even, then Lemma 4.1 gives $y^{0}=0$, and therefore (4.31) implies (4.32).

(2). If $1 \leq m \leq 2+p$ and $m$ is odd, then Proposition 4.3(2) gives (4.33). Moreover, it yields from (4.2) that $x_{k}$ satisfies $\left|\frac{x_{k}}{\varepsilon_{k}}\right|=O\left(\varepsilon_{k}^{m}\left|y^{0}\right|\right)+o\left(\varepsilon_{k}^{m}\right)$ as $k \rightarrow \infty$, which then implies (4.34) for some $y^{0} \in \mathbb{R}^{2}$.

(3). If $m=2+p$ is even, then Proposition 4.3(4) gives (4.35), and we reduce from (4.1) that $x_{k}$ satisfies (4.32).

(4). If $1 \leq m<2+p$ and $m$ is even, it then follows from (4.1) that $x_{k}$ always satisfies (4.36). Moreover, Proposition 4.3)(3) gives that if $\mathcal{S}=0$, then $u_{k}$ satisfies (4.33); if $\mathcal{S} \neq 0$, then $u_{k}$ satisfies (4.37).

\section{A Appendix: The Proof of Lemma 3.4}

In this appendix, we shall follow Lemmas 3.1 and 3.3 to address the proof of Lemma 3.4 . i.e., (3.34)-(3.36).

The proof of (3.34). Under the assumptions of Lemma 3.4, we first note that the equation (3.7) can be simplified as

$$
\nabla \psi_{1}(0)=0, \quad \mathcal{L} \psi_{1}=-\frac{2 w^{3}}{\int_{\mathbb{R}^{2}} w^{4}}-\frac{2 h\left(x+y_{0}\right) w}{p \int_{\mathbb{R}^{2}} h\left(x+y_{0}\right) w^{2}} \text { in } \mathbb{R}^{2},
$$

due to the fact that

$$
a^{*}=\|w\|_{2}^{2}=\frac{1}{2} \int_{\mathbb{R}^{2}} w^{4} .
$$

By (1.14), (3.10) and (A.1), we then have

$$
\begin{aligned}
2 \int_{\mathbb{R}^{2}} w \psi_{1} & =2 \int_{\mathbb{R}^{2}} \mathcal{L} \psi_{2} \psi_{1}=2 \int_{\mathbb{R}^{2}} \psi_{2} \mathcal{L} \psi_{1} \\
& =\int_{\mathbb{R}^{2}}\left[\frac{2 w^{3}}{\int_{\mathbb{R}^{2}} w^{4}}+\frac{2 h\left(x+y_{0}\right) w}{p \int_{\mathbb{R}^{2}} h\left(x+y_{0}\right) w^{2}}\right](w+x \cdot \nabla w) \\
& =2+\frac{2}{p}+\frac{2}{\int_{\mathbb{R}^{2}} w^{4}} \int_{\mathbb{R}^{2}} w^{3}(x \cdot \nabla w)+\frac{2}{p \int_{\mathbb{R}^{2}} h\left(x+y_{0}\right) w^{2}} \int_{\mathbb{R}^{2}} h\left(x+y_{0}\right) w(x \cdot \nabla w) \\
& =2+\frac{2}{p}+\frac{1}{2 \int_{\mathbb{R}^{2}} w^{4}} \int_{\mathbb{R}^{2}}\left(x \cdot \nabla w^{4}\right)+\frac{1}{p \int_{\mathbb{R}^{2}} h\left(x+y_{0}\right) w^{2}} \int_{\mathbb{R}^{2}} h\left(x+y_{0}\right)\left(x \cdot \nabla w^{2}\right) \\
& =2+\frac{2}{p}-1-\frac{1}{p \int_{\mathbb{R}^{2}} h\left(x+y_{0}\right) w^{2}} \int_{\mathbb{R}^{2}} w^{2}\left[2 h\left(x+y_{0}\right)+\left(x \cdot \nabla h\left(x+y_{0}\right)\right)\right] \\
& =2+\frac{2}{p}-1-\frac{(p+2)}{p}=0,
\end{aligned}
$$

since $\left(x+y_{0}\right) \cdot \nabla h\left(x+y_{0}\right)=p h\left(x+y_{0}\right)$ and $\int_{\mathbb{R}^{2}} w^{2}\left[y_{0} \cdot \nabla h\left(x+y_{0}\right)\right]=0$. Also, we deduce from (3.10) that

$$
2 \int_{\mathbb{R}^{2}} w \psi_{2}=-\int_{\mathbb{R}^{2}} w(w+x \cdot \nabla w)=-\int_{\mathbb{R}^{2}} w^{2}-\frac{1}{2} \int_{\mathbb{R}^{2}}\left(x \cdot \nabla w^{2}\right)=0
$$


which thus completes the proof of (3.34).

The proof of (3.35). By Lemmas 3.1 and 3.3, we obtain that

$$
\begin{aligned}
I & =\int_{\mathbb{R}^{2}}\left(2 w \psi_{4}+\psi_{2}^{2}\right)=\int_{\mathbb{R}^{2}} \psi_{2}^{2}+2\left\langle\mathcal{L} \psi_{2}, \psi_{4}\right\rangle \\
& =\int_{\mathbb{R}^{2}} \psi_{2}^{2}+2\left\langle\psi_{2}, \mathcal{L} \psi_{4}\right\rangle=\int_{\mathbb{R}^{2}} \psi_{2}^{2}+2\left\langle\psi_{2},\left(3 w \psi_{2}^{2}+\psi_{2}\right)\right\rangle \\
& =3 \int_{\mathbb{R}^{2}} \psi_{2}^{2}+6 \int_{\mathbb{R}^{2}} w \psi_{2}^{3},
\end{aligned}
$$

which implies that

$$
\begin{aligned}
\frac{4 I}{3} \div 2 \pi & =4\left[\int_{\mathbb{R}^{2}} \psi_{2}^{2}+2 \int_{\mathbb{R}^{2}} w \psi_{2}^{3}\right] \div 2 \pi \\
& =\int_{0}^{\infty} r\left(w+r w^{\prime}\right)^{2}-\int_{0}^{\infty} r w\left(w+r w^{\prime}\right)^{3}:=A-B .
\end{aligned}
$$

Here we have

$$
\begin{aligned}
A=\int_{0}^{\infty} r\left(w+r w^{\prime}\right)^{2} & =\int_{0}^{\infty} r^{3}\left(w^{\prime}\right)^{2}+\int_{0}^{\infty} r w^{2}+\int_{0}^{\infty} r^{2} d w^{2} \\
& =\int_{0}^{\infty} r^{3}\left(w^{\prime}\right)^{2}-\frac{1}{2} \int_{0}^{\infty} r w^{4}
\end{aligned}
$$

where (A.2) is used, and

$$
\begin{aligned}
B & =\int_{0}^{\infty} r w\left(w+r w^{\prime}\right)^{3} \\
& =\left[\int_{0}^{\infty} r w^{4}+3 \int_{0}^{\infty} r^{2} w^{3} w^{\prime}\right]+3 \int_{0}^{\infty} r^{3} w^{2} w^{\prime} w^{\prime}+\int_{0}^{\infty} r^{4} w\left(w^{\prime}\right)^{3} \\
& =-\frac{1}{2} \int_{0}^{\infty} r w^{4}+3 \int_{0}^{\infty} r^{3} w^{2} w^{\prime} w^{\prime}+\int_{0}^{\infty} r^{4} w\left(w^{\prime}\right)^{3} .
\end{aligned}
$$

Therefore, we get from (A.3) that

$$
\frac{4 I}{3} \div 2 \pi=\int_{0}^{\infty} r^{3}\left(w^{\prime}\right)^{2}-\int_{0}^{\infty} r^{4} w\left(w^{\prime}\right)^{3}-3 \int_{0}^{\infty} r^{3} w^{2} w^{\prime} w^{\prime}:=C+D+E .
$$

To further simplify $I$, recall that

$$
r w^{\prime \prime}=-w^{\prime}+r w-r w^{3},
$$

by which we then have

$$
\begin{aligned}
C & =\int_{0}^{\infty} r^{3} w^{\prime} d w=-\int_{0}^{\infty} w\left(r^{3} w^{\prime}\right)^{\prime} \\
& =-\int_{0}^{\infty} w\left[3 r^{2} w^{\prime}+r^{2}\left(-w^{\prime}+r w-r w^{3}\right)\right] \\
& =-\int_{0}^{\infty} w\left[2 r^{2} w^{\prime}+r^{3} w-r^{3} w^{3}\right] \\
& =2 \int_{0}^{\infty} r w^{2}-\int_{0}^{\infty} r^{3} w^{2}+\int_{0}^{\infty} r^{3} w^{4}
\end{aligned}
$$


Similarly, we have

$$
\begin{aligned}
D=-\frac{1}{2} \int_{0}^{\infty} r^{4}\left(w^{\prime}\right)^{2} d w^{2} & =\frac{1}{2} \int_{0}^{\infty} w^{2}\left[4 r^{3}\left(w^{\prime}\right)^{2}+2 r^{3} w^{\prime}\left(-w^{\prime}+r w-r w^{3}\right)\right] \\
& =\int_{0}^{\infty} r^{3} w^{2}\left(w^{\prime}\right)^{2}+\frac{1}{4} \int_{0}^{\infty} r^{4} d w^{4}-\frac{1}{6} \int_{0}^{\infty} r^{4} d w^{6} \\
& =\int_{0}^{\infty} r^{3} w^{2}\left(w^{\prime}\right)^{2}-\int_{0}^{\infty} r^{3} w^{4}+\frac{2}{3} \int_{0}^{\infty} r^{3} w^{6}
\end{aligned}
$$

Note from (A.5) that

$$
\begin{aligned}
-2 \int_{0}^{\infty} r^{3} w^{2}\left(w^{\prime}\right)^{2} & =-\frac{2}{3} \int_{0}^{\infty} r^{3} w^{\prime} d w^{3}=\frac{2}{3} \int_{0}^{\infty} w^{3}\left[3 r^{2} w^{\prime}+r^{2}\left(-w^{\prime}+r w-r w^{3}\right)\right] \\
& =\frac{4}{3} \int_{0}^{\infty} w^{3} r^{2} w^{\prime}+\frac{2}{3} \int_{0}^{\infty} r^{3} w^{4}-\frac{2}{3} \int_{0}^{\infty} r^{3} w^{6} \\
& =-\frac{2}{3} \int_{0}^{\infty} r w^{4}+\frac{2}{3} \int_{0}^{\infty} r^{3} w^{4}-\frac{2}{3} \int_{0}^{\infty} r^{3} w^{6} .
\end{aligned}
$$

We thus derive that

$$
\begin{aligned}
D+E & =-2 \int_{0}^{\infty} r^{3} w^{2}\left(w^{\prime}\right)^{2}-\int_{0}^{\infty} r^{3} w^{4}+\frac{2}{3} \int_{0}^{\infty} r^{3} w^{6} \\
& =-\frac{2}{3} \int_{0}^{\infty} r w^{4}-\frac{1}{3} \int_{0}^{\infty} r^{3} w^{4},
\end{aligned}
$$

by which we conclude from (A.2) and (A.4) that

$$
\frac{4 I}{3} \div 2 \pi=C+D+E=\frac{1}{3}\left[2 \int_{0}^{\infty} r w^{2}-3 \int_{0}^{\infty} r^{3} w^{2}+2 \int_{0}^{\infty} r^{3} w^{4}\right] .
$$

In the following, we note that $w$ satisfies

$$
\left(r w^{\prime}\right)^{\prime}=r w-r w^{3}, \quad r>0 .
$$

Multiplying (A.7) by $r^{3} w^{\prime}$ and integrating on $[0, \infty)$, we get that

$$
\begin{aligned}
\int_{0}^{\infty} r^{3} w^{\prime}\left(r w^{\prime}\right)^{\prime} & =\int_{0}^{\infty} r^{3} w^{\prime}\left[r w-r w^{3}\right]=\frac{1}{2} \int_{0}^{\infty} r^{4} d w^{2}-\frac{1}{4} \int_{0}^{\infty} r^{4} d w^{4} \\
& =-2 \int_{0}^{\infty} r^{3} w^{2}+\int_{0}^{\infty} r^{3} w^{4}
\end{aligned}
$$

Note also that

$$
\int_{0}^{\infty} r^{3} w^{\prime}\left(r w^{\prime}\right)^{\prime}=\int_{0}^{\infty} r^{3}\left(w^{\prime}\right)^{2}+\frac{1}{2} \int_{0}^{\infty} r^{4} d\left(w^{\prime}\right)^{2}=-\int_{0}^{\infty} r^{3}\left(w^{\prime}\right)^{2} .
$$

By combining above two identities, it yields that

$$
\int_{0}^{\infty} r^{3}\left(w^{\prime}\right)^{2}=2 \int_{0}^{\infty} r^{3} w^{2}-\int_{0}^{\infty} r^{3} w^{4} .
$$


On the other hand, multiplying (A.7) by $r^{2} w$ and integrating on $[0, \infty)$, we obtain that

$$
\begin{aligned}
\int_{0}^{\infty} r^{3} w^{2}-\int_{0}^{\infty} r^{3} w^{4} & =\int_{0}^{\infty} r^{2} w\left(r w^{\prime}\right)^{\prime}=\int_{0}^{\infty} r^{2} w w^{\prime}+\int_{0}^{\infty} r^{3} w d w^{\prime} \\
& =\int_{0}^{\infty} r^{2} w w^{\prime}-\int_{0}^{\infty} w^{\prime}\left(3 r^{2} w+r^{3} w^{\prime}\right) \\
& =-2 \int_{0}^{\infty} r^{2} w w^{\prime}-\int_{0}^{\infty} r^{3}\left(w^{\prime}\right)^{2} \\
& =2 \int_{0}^{\infty} r w^{2}-\int_{0}^{\infty} r^{3}\left(w^{\prime}\right)^{2},
\end{aligned}
$$

which then implies that

$$
\int_{0}^{\infty} r^{3}\left(w^{\prime}\right)^{2}=2 \int_{0}^{\infty} r w^{2}-\int_{0}^{\infty} r^{3} w^{2}+\int_{0}^{\infty} r^{3} w^{4}
$$

We thus conclude from (A.8) and (A.9) that

$$
2 \int_{0}^{\infty} r w^{2}-3 \int_{0}^{\infty} r^{3} w^{2}+2 \int_{0}^{\infty} r^{3} w^{4}=0
$$

which therefore implies that $I=0$ in view of (A.6), i.e., (3.35) holds.

The proof of (3.36). Following Lemmas 3.1 and 3.3 again, we get that

$$
\begin{aligned}
I I= & 2 \int_{\mathbb{R}^{2}} \psi_{5} \mathcal{L} \psi_{2}+2 \int_{\mathbb{R}^{2}} \psi_{1} \psi_{2}=2 \int_{\mathbb{R}^{2}} \psi_{2}\left[\mathcal{L} \psi_{5}+\psi_{1}\right] \\
= & -\int_{\mathbb{R}^{2}}(w+x \cdot \nabla w)\left(6 w \psi_{1} \psi_{2}+2 \psi_{1}\right) \\
& -\frac{1}{2} \int_{\mathbb{R}^{2}}\left[\frac{3 w^{2}}{a^{*}}+\frac{h\left(x+y_{0}\right)}{\lambda^{2+p}}\right](w+x \cdot \nabla w)^{2} \\
& +\frac{1}{2 \lambda^{2+p}} \int_{\mathbb{R}^{2}}(w+x \cdot \nabla w)\left[y_{0} \cdot \nabla h\left(x+y_{0}\right)\right] w \\
:= & A+B .
\end{aligned}
$$

Since $\left(x+y_{0}\right) \cdot \nabla h\left(x+y_{0}\right)=p h\left(x+y_{0}\right)$ holds in $\mathbb{R}^{2}$, we derive from (1.14) and (A.1) that

$$
\begin{aligned}
B= & -\frac{1}{2} \int_{\mathbb{R}^{2}}\left[\frac{3 w^{2}}{a^{*}}+\frac{h\left(x+y_{0}\right)}{\lambda^{2+p}}\right]\left[w^{2}+2 w(x \cdot \nabla w)+(x \cdot \nabla w)^{2}\right] \\
& +\frac{1}{2 \lambda^{2+p}} \int_{\mathbb{R}^{2}}(w+x \cdot \nabla w)\left[y_{0} \cdot \nabla h\left(x+y_{0}\right)\right] w \\
= & -\int_{\mathbb{R}^{2}}\left[\frac{3 w^{2}}{\int_{\mathbb{R}^{2}} w^{4}}+\frac{h\left(x+y_{0}\right)}{p \int_{\mathbb{R}^{2}} h\left(x+y_{0}\right) w^{2}}\right]\left[w^{2}+2 w(x \cdot \nabla w)\right] \\
& -\frac{1}{2} \int_{\mathbb{R}^{2}}\left[\frac{3 w^{2}}{a^{*}}+\frac{h\left(x+y_{0}\right)}{\lambda^{2+p}}\right](x \cdot \nabla w)^{2}+\frac{1}{2 \lambda^{2+p}} \int_{\mathbb{R}^{2}} w\left[y_{0} \cdot \nabla h\left(x+y_{0}\right)\right](x \cdot \nabla w) \\
= & -3-\frac{1}{p}-\frac{3}{2 \int_{\mathbb{R}^{2}} w^{4}} \int_{\mathbb{R}^{2}}\left(x \cdot \nabla w^{4}\right)-\frac{1}{p \int_{\mathbb{R}^{2}} h\left(x+y_{0}\right) w^{2}} \int_{\mathbb{R}^{2}} h\left(x+y_{0}\right)\left(x \cdot \nabla w^{2}\right) \\
& -\frac{1}{2} \int_{\mathbb{R}^{2}}\left[\frac{3 w^{2}}{a^{*}}+\frac{h\left(x+y_{0}\right)}{\lambda^{2+p}}\right](x \cdot \nabla w)^{2}+\frac{1}{2 \lambda^{2+p}} \int_{\mathbb{R}^{2}} w\left[y_{0} \cdot \nabla h\left(x+y_{0}\right)\right](x \cdot \nabla w) \\
:= & -3-\frac{1}{p}+3+\frac{2+p}{p}+C_{0}=\frac{p+1}{p}+C_{0},
\end{aligned}
$$


where the term $C_{0}$ satisfies

$$
\begin{aligned}
C_{0}= & -\frac{1}{2} \int_{\mathbb{R}^{2}}\left[\frac{3 w^{2}}{a^{*}}+\frac{h\left(x+y_{0}\right)}{\lambda^{2+p}}\right](x \cdot \nabla w)^{2}+\frac{1}{2 \lambda^{2+p}} \int_{\mathbb{R}^{2}} w\left[y_{0} \cdot \nabla h\left(x+y_{0}\right)\right](x \cdot \nabla w) \\
= & -\frac{1}{2 a^{*}} \int_{\mathbb{R}^{2}}(x \cdot \nabla w)\left(x \cdot \nabla w^{3}\right)-\frac{1}{2 \lambda^{2+p}} \int_{\mathbb{R}^{2}} h\left(x+y_{0}\right)(x \cdot \nabla w)(x \cdot \nabla w) \\
& +\frac{1}{2 \lambda^{2+p}} \int_{\mathbb{R}^{2}} w\left[y_{0} \cdot \nabla h\left(x+y_{0}\right)\right](x \cdot \nabla w) \\
= & \frac{1}{2 a^{*}} \int_{\mathbb{R}^{2}} w^{3}[2(x \cdot \nabla w)+x \cdot \nabla(x \cdot \nabla w)] \\
& +\frac{1}{2 \lambda^{2+p}} \int_{\mathbb{R}^{2}} w\left\{2 h\left(x+y_{0}\right)(x \cdot \nabla w)+\left[x \cdot \nabla h\left(x+y_{0}\right)\right](x \cdot \nabla w)\right. \\
= & \frac{1}{2} \int_{\mathbb{R}^{2}}\left[\frac{w^{3}}{a^{*}}+\frac{w h\left(x+y_{0}\right)}{\lambda^{2+p}}\right][x \cdot \nabla(x \cdot \nabla w)] \\
& +\frac{1}{a^{*}} \int_{\mathbb{R}^{2}} w^{3}(x \cdot \nabla w)+\frac{2+p}{2 \lambda^{2+p}} \int_{\mathbb{R}^{2}} w h\left(x+y_{0}\right)(x \cdot \nabla w) \\
= & \frac{1}{2} \int_{\mathbb{R}^{2}}\left[\frac{w^{3}}{a^{*}}+\frac{w h\left(x+y_{0}\right)}{\lambda^{2+p}}\right][x \cdot \nabla(x \cdot \nabla w)] \\
& \left.+\frac{1}{2 \int_{\mathbb{R}^{2}} w^{4}} \int_{\mathbb{R}^{2}}\left(x \cdot \nabla w^{4}\right)+\frac{2+p}{2 p \int_{\mathbb{R}^{2}} h\left(x+y_{0}\right) y^{2}} \cdot \nabla h\left(x+y_{0}\right)\right](x \cdot \nabla w) \\
= & \frac{1}{2} \int_{\mathbb{R}^{2}}\left[\frac{w^{3}}{a^{*}}+\frac{w h\left(x+y_{0}\right)}{\lambda^{2+p}}\right][x \cdot \nabla(x \cdot \nabla w)]-1-\frac{(2+p)^{2}}{2 p}, \\
& \left.+y_{0}\right)\left(x \cdot \nabla w^{2}\right)
\end{aligned}
$$

in view of (A.1). We thus have

$$
B=\frac{1}{2} \int_{\mathbb{R}^{2}}\left[\frac{w^{3}}{a^{*}}+\frac{w h\left(x+y_{0}\right)}{\lambda^{2+p}}\right][x \cdot \nabla(x \cdot \nabla w)]-\frac{p^{2}+4 p+2}{2 p} .
$$

We next calculate the term $A$ as follows. Observe that

$$
\begin{aligned}
& \frac{1}{2} \int_{\mathbb{R}^{2}} \psi_{1} x \cdot \nabla(x \cdot \nabla w) \\
= & -\frac{1}{2} \int_{\mathbb{R}^{2}}(x \cdot \nabla w)\left[2 \psi_{1}+\left(x \cdot \nabla \psi_{1}\right)\right] \\
= & -\int_{\mathbb{R}^{2}} \psi_{1}(x \cdot \nabla w)-\frac{1}{2} \int_{\mathbb{R}^{2}}\left(x \cdot \nabla \psi_{1}\right)(x \cdot \nabla w) \\
= & -\int_{\mathbb{R}^{2}} \psi_{1}(x \cdot \nabla w)+\frac{1}{2} \int_{\mathbb{R}^{2}} w\left[2\left(x \cdot \nabla \psi_{1}\right)+x \cdot \nabla\left(x \cdot \nabla \psi_{1}\right)\right] \\
= & -\int_{\mathbb{R}^{2}} \psi_{1}(x \cdot \nabla w)+\int_{\mathbb{R}^{2}} w\left(x \cdot \nabla \psi_{1}\right)+\frac{1}{2} \int_{\mathbb{R}^{2}} w x \cdot \nabla\left(x \cdot \nabla \psi_{1}\right),
\end{aligned}
$$

which implies that

$$
\begin{aligned}
& -\int_{\mathbb{R}^{2}} \psi_{1}(x \cdot \nabla w)+\int_{\mathbb{R}^{2}} w\left(x \cdot \nabla \psi_{1}\right) \\
= & \frac{1}{2} \int_{\mathbb{R}^{2}} \psi_{1} x \cdot \nabla(x \cdot \nabla w)-\frac{1}{2} \int_{\mathbb{R}^{2}} w x \cdot \nabla\left(x \cdot \nabla \psi_{1}\right) .
\end{aligned}
$$


Using (A.12), we then derive that

$$
\begin{aligned}
A= & -\int_{\mathbb{R}^{2}}(w+x \cdot \nabla w)\left(6 w \psi_{1} \psi_{2}+2 \psi_{1}\right) \\
= & -2 \int_{\mathbb{R}^{2}} w \psi_{1}-2 \int_{\mathbb{R}^{2}} \psi_{1}(x \cdot \nabla w)+3 \int_{\mathbb{R}^{2}} w \psi_{1}(w+x \cdot \nabla w)^{2} \\
= & -2 \int_{\mathbb{R}^{2}} w \psi_{1}-\int_{\mathbb{R}^{2}} \psi_{1}(x \cdot \nabla w) \\
& +\int_{\mathbb{R}^{2}} w\left[2 \psi_{1}+x \cdot \nabla \psi_{1}\right]+3 \int_{\mathbb{R}^{2}} w \psi_{1}(w+x \cdot \nabla w)^{2} \\
= & -\int_{\mathbb{R}^{2}} \psi_{1}(x \cdot \nabla w)+\int_{\mathbb{R}^{2}} w\left(x \cdot \nabla \psi_{1}\right)+D \\
= & \frac{1}{2} \int_{\mathbb{R}^{2}} \psi_{1} x \cdot \nabla(x \cdot \nabla w)-\frac{1}{2} \int_{\mathbb{R}^{2}} w x \cdot \nabla\left(x \cdot \nabla \psi_{1}\right)+D
\end{aligned}
$$

where the term $D$ satisfies

$$
\begin{aligned}
D= & 3 \int_{\mathbb{R}^{2}} w \psi_{1}\left[w^{2}+2 w(x \cdot \nabla w)+(x \cdot \nabla w)^{2}\right] \\
= & 3 \int_{\mathbb{R}^{2}} w^{3} \psi_{1}+6 \int_{\mathbb{R}^{2}} w^{2} \psi_{1}(x \cdot \nabla w)+\frac{3}{2} \int_{\mathbb{R}^{2}} \psi_{1}(x \cdot \nabla w)\left(x \cdot \nabla w^{2}\right) \\
= & 3 \int_{\mathbb{R}^{2}} w^{3} \psi_{1}+6 \int_{\mathbb{R}^{2}} w^{2} \psi_{1}(x \cdot \nabla w) \\
& -\frac{3}{2} \int_{\mathbb{R}^{2}} w^{2}\left\{2 \psi_{1}(x \cdot \nabla w)+(x \cdot \nabla w)\left(x \cdot \nabla \psi_{1}\right)+\psi_{1}[x \cdot \nabla(x \cdot \nabla w)]\right\} .
\end{aligned}
$$

Since

$$
\begin{aligned}
& -\frac{3}{2} \int_{\mathbb{R}^{2}} w^{2}(x \cdot \nabla w)\left(x \cdot \nabla \psi_{1}\right) \\
= & -\frac{1}{2} \int_{\mathbb{R}^{2}}\left(x \cdot \nabla \psi_{1}\right)\left(x \cdot \nabla w^{3}\right) \\
= & \frac{1}{2} \int_{\mathbb{R}^{2}} w^{3}\left[x \cdot \nabla\left(x \cdot \nabla \psi_{1}\right)+2\left(x \cdot \nabla \psi_{1}\right)\right] \\
= & \frac{1}{2} \int_{\mathbb{R}^{2}} w^{3} x \cdot \nabla\left(x \cdot \nabla \psi_{1}\right)+\int_{\mathbb{R}^{2}} w^{3}\left(x \cdot \nabla \psi_{1}\right) \\
= & \frac{1}{2} \int_{\mathbb{R}^{2}} w^{3} x \cdot \nabla\left(x \cdot \nabla \psi_{1}\right)-\int_{\mathbb{R}^{2}} \psi_{1}\left[2 w^{3}+3 w^{2}(x \cdot \nabla w)\right] \\
= & \frac{1}{2} \int_{\mathbb{R}^{2}} w^{3} x \cdot \nabla\left(x \cdot \nabla \psi_{1}\right)-2 \int_{\mathbb{R}^{2}} w^{3} \psi_{1}-3 \int_{\mathbb{R}^{2}} w^{2} \psi_{1}(x \cdot \nabla w),
\end{aligned}
$$

the term $D$ can be further simplified as

$$
D=\int_{\mathbb{R}^{2}} w^{3} \psi_{1}-\frac{3}{2} \int_{\mathbb{R}^{2}} w^{2} \psi_{1}[x \cdot \nabla(x \cdot \nabla w)]+\frac{1}{2} \int_{\mathbb{R}^{2}} w^{3} x \cdot \nabla\left(x \cdot \nabla \psi_{1}\right) .
$$

Applying (A.14), we then obtain from (A.13) that

$$
\begin{aligned}
A= & \int_{\mathbb{R}^{2}} w^{3} \psi_{1}+\frac{1}{2} \int_{\mathbb{R}^{2}}\left(1-3 w^{2}\right) \psi_{1}[x \cdot \nabla(x \cdot \nabla w)] \\
& -\frac{1}{2} \int_{\mathbb{R}^{2}} \Delta w\left[x \cdot \nabla\left(x \cdot \nabla \psi_{1}\right)\right]
\end{aligned}
$$


since $w$ solves the equation $w^{3}-w=-\Delta w$ in $\mathbb{R}^{2}$.

Combining (A.11) and (A.15) now yields that

$$
\begin{aligned}
I I=A+B= & \int_{\mathbb{R}^{2}} w^{3} \psi_{1}-\frac{p^{2}+4 p+2}{2 p} \\
& +\frac{1}{2} \int_{\mathbb{R}^{2}}[x \cdot \nabla(x \cdot \nabla w)] \Delta \psi_{1}-\frac{1}{2} \int_{\mathbb{R}^{2}}\left[x \cdot \nabla\left(x \cdot \nabla \psi_{1}\right)\right] \Delta w .
\end{aligned}
$$

We claim that

$$
\int_{\mathbb{R}^{2}} w^{3} \psi_{1}=\frac{p+1}{p}
$$

Actually, multiplying (A.1) by $w$ and integrating on $\mathbb{R}^{2}$ gives that

$$
\int_{\mathbb{R}^{2}} \nabla \psi_{1} \nabla w-3 \int_{\mathbb{R}^{2}} w^{3} \psi_{1}=-\int_{\mathbb{R}^{2}}\left[\frac{2 w^{4}}{\int_{\mathbb{R}^{2}} w^{4}}+\frac{2 h\left(x+y_{0}\right) w^{2}}{p \int_{\mathbb{R}^{2}} h\left(x+y_{0}\right) w^{2}}\right]=-\frac{2(p+1)}{p},
$$

due to the fact that $\int_{\mathbb{R}^{2}} w \psi_{1}=0$ by (3.34). On the other hand, multiplying (1.4) by $\psi_{1}$ and integrating on $\mathbb{R}^{2}$ gives that

$$
\int_{\mathbb{R}^{2}} \nabla \psi_{1} \nabla w=-\int_{\mathbb{R}^{2}} w \psi_{1}+\int_{\mathbb{R}^{2}} w^{3} \psi_{1}=\int_{\mathbb{R}^{2}} w^{3} \psi_{1} .
$$

The claim (A.17) then follows directly from above two identities. We next claim that

$$
\int_{\mathbb{R}^{2}}[x \cdot \nabla(x \cdot \nabla w)] \Delta \psi_{1}=\int_{\mathbb{R}^{2}}\left[x \cdot \nabla\left(x \cdot \nabla \psi_{1}\right)\right] \Delta w .
$$

To prove (A.18), rewrite $\psi_{1}$ as $\psi_{1}(x)=\psi_{1}(r, \theta)$, where $(r, \theta)$ is the polar coordinate in $\mathbb{R}^{2}$, such that

$$
\Delta \psi_{1}=\left(\psi_{1}\right)_{r r}+\frac{1}{r}\left(\psi_{1}\right)_{r}+\frac{1}{r^{2}}\left(\psi_{1}\right)_{\theta \theta}, \quad \nabla \psi_{1}=\frac{x}{r}\left(\psi_{1}\right)_{r}+\frac{x^{\perp}}{r^{2}}\left(\psi_{1}\right)_{\theta},
$$

where $x^{\perp}=\left(-x_{2}, x_{1}\right)$ for $x=\left(x_{1}, x_{2}\right) \in \mathbb{R}^{2}$. We then derive from (3.7) that

$$
\begin{aligned}
\int_{\mathbb{R}^{2}}[x \cdot \nabla(x \cdot \nabla w)] \Delta \psi_{1} & =\int_{0}^{2 \pi} \int_{0}^{\infty} r\left(r w^{\prime}\right)^{\prime}\left\{\left[r\left(\psi_{1}\right)_{r}\right]_{r}+\frac{\left(\psi_{1}\right)_{\theta \theta}}{r}\right\} d r d \theta \\
& =\int_{0}^{2 \pi} \int_{0}^{\infty} r\left(r w^{\prime}\right)^{\prime}\left[r\left(\psi_{1}\right)_{r}\right]_{r} d r d \theta+\int_{0}^{2 \pi} \int_{0}^{\infty}\left(r w^{\prime}\right)^{\prime}\left(\psi_{1}\right)_{\theta \theta} d r d \theta \\
& =\int_{0}^{2 \pi} \int_{0}^{\infty} r\left(r w^{\prime}\right)^{\prime}\left[r\left(\psi_{1}\right)_{r}\right]_{r} d r d \theta
\end{aligned}
$$

and

$$
\int_{\mathbb{R}^{2}}\left[x \cdot \nabla\left(x \cdot \nabla \psi_{1}\right)\right] \Delta w=\int_{0}^{2 \pi} \int_{0}^{\infty} r\left[r\left(\psi_{1}\right)_{r}\right]_{r}\left(r w^{\prime}\right)^{\prime} d r d \theta
$$

which thus imply that (A.18) holds. Applying (A.17) and (A.18), we therefore conclude from (A.16) that

$$
I I=\frac{p+1}{p}-\frac{p^{2}+4 p+2}{2 p}=-\frac{2+p}{2}
$$

which gives (3.36), and the proof is complete. 
Acknowledgements: The authors are very grateful to the referees for many useful suggestions which lead to some improvements of the present paper. The first author thanks Prof. Robert Seiringer for fruitful discussions on the present work. Part of the present work was finished when the first author was visiting Taida Institute of Mathematical Sciences (TIMS) in October 2013 and Pacific Institute for Mathematical Sciences (PIMS) from March to April in 2016. He would like to thank both institutes for their warm hospitality.

\section{References}

[1] M. H. Anderson, J. R. Ensher, M. R. Matthews, C. E. Wieman and E. A. Cornell, Observation of Bose-Einstein condensation in a dilute atomic vapor, Science 269 (1995), 198-201.

[2] W. Z. Bao and Y. Y. Cai, Mathematical theory and numerical methods for BoseEinstein condensation, Kinetic and Related Models 6 (2013), 1-135.

[3] D. M. Cao, S. L. Li and P. Luo, Uniqueness of positive bound states with multibump for nonlinear Schrödinger equations, Calc. Var. Partial Differential Equations 54 (2015), no. 4, 4037-4063.

[4] T. Cazenave, Semilinear Schrödinger Equations, Courant Lecture Notes in Math. 10, Courant Institute of Mathematical Science/AMS, New York, (2003).

[5] F. Dalfovo, S. Giorgini, L. P. Pitaevskii and S. Stringari, Theory of Bose-Einstein condensation in trapped gases, Rev. Modern Phys. 71 (1999), 463-512.

[6] Y. B. Deng, C. S. Lin and S. Yan, On the prescribed scalar curvature problem in $\mathbb{R}^{N}$, local uniqueness and periodicity, J. Math. Pures Appl. 104 (2015), no. 6, 1013-1044.

[7] B. Gidas, W. M. Ni and L. Nirenberg, Symmetry of positive solutions of nonlinear elliptic equations in $\mathbb{R}^{n}$, Mathematical analysis and applications Part A, Adv. in Math. Suppl. Stud. vol. 7 (1981), 369-402.

[8] D. Gilbarg and N. S. Trudinger, Elliptic Partial Differential Equations of Second Order, Springer, (1997).

[9] M. Grossi, On the number of single-peak solutions of the nonlinear Schrödinger equations, Ann. Inst H. Poincar Anal. Non Linaire 19 (2002), 261-280.

[10] Y. J. Guo and R. Seiringer, On the mass concentration for Bose-Einstein condensates with attractive interactions, Lett. Math. Phys. 104 (2014), 141-156.

[11] Y. J. Guo, Z. Q. Wang, X. Y. Zeng and H. S. Zhou, Properties for ground states of attractive Gross-Pitaevskii equations with multi-well potentials, arXiv:1502.01839, submitted, (2015).

[12] Y. J. Guo, X. Y. Zeng and H. S. Zhou, Energy estimates and symmetry breaking in attractive Bose-Einstein condensates with ring-shaped potentials, Ann. Inst. H. Poincaré Anal. Non Linéaire 33 (2016), 809-828. 
[13] Y. Kagan, A. E. Muryshev and G. V. Shlyapnikov, Collapse and Bose-Einstein condensation in a trapped Bose gas with nagative scattering length, Phys. Rev. Lett. 81 (1998), 933-937.

[14] M. K. Kwong, Uniqueness of positive solutions of $\Delta u-u+u^{p}=0$ in $\mathbb{R}^{N}$, Arch. Rational Mech. Anal. 105 (1989), 243-266.

[15] E. H. Lieb and M. Loss, Analysis, Graduate Studies in Math. 14, Amer. Math. Soc., Providence, RI, second edition (2001).

[16] T. C. Lin and J. C. Wei, Orbital stability of bound states of semiclassical nonlinear Schrödinger equations with critical nonlinearity, SIAM J. Math. Anal. 40 (2008), no. $1,365-381$.

[17] T. C. Lin, J. C. Wei and W. Yao, Orbital stability of bound states of nonlinear Schrödinger equations with linear and nonlinear optical lattices, J. Differential Equations 249 (2010), no. 9, 2111-2146.

[18] M. Maeda, On the symmetry of the ground states of nonlinear Schrödinger equation with potential, Adv. Nonlinear Stud. 10 (2010), 895-925.

[19] K. McLeod and J. Serrin, Uniqueness of positive radial solutions of $\Delta u+f(u)=0$ in $\mathbb{R}^{n}$, Arch. Rational Mech. Anal. 99 (1987), 115-145.

[20] W.-M. Ni and I. Takagi, On the shape of least-energy solutions to a semilinear Neumann problem, Comm. Pure Appl. Math. 44 (1991), 819-851.

[21] W.-M. Ni and J. C. Wei, On the location and profile of spike-layer solutions to singularly perturbed semilinear Dirichlet problems, Comm. Pure Appl. Math. 48 (1995), 731-768.

[22] M. Reed and B. Simon, Methods of Modern Mathematical Physics. IV. Analysis of Operators, Academic Press, New York-London, 1978.

[23] C. A. Sackett, H. T. C. Stoof and R. G. Hulet, Growth and collapse of a BoseEinstein condensate with attractive interactions, Phys. Rev. Lett. 80 (1998), 2031.

[24] X. F. Wang, On concentration of positive bound states of nonlinear Schrödinger equations, Comm. Math. Phys. 153 (1993), 229-244.

[25] M. I. Weinstein, Nonlinear Schrödinger equations and sharp interpolations estimates, Comm. Math. Phys. 87 (1983), 567-576.

[26] J. Zhang, Stability of attractive Bose-Einstein condensates, J. Stat. Phys. 101 (2000), 731-746. 